Canad. J. Math. Vol. 71 (4), 2019 pp. 891-935

http://dx.doi.org/10.4153/CJM-2018-020-0

(C) Canadian Mathematical Society 2018

\title{
Cohomological Approach to Class Field Theory in Arithmetic Topology
}

\author{
Tomoki Mihara
}

\begin{abstract}
We establish class field theory for three-dimensional manifolds and knots. For this purpose, we formulate analogues of the multiplicative group, the idèle class group, and ray class groups in a cocycle-theoretic way. Following the arguments in abstract class field theory, we construct reciprocity maps and verify the existence theorems.
\end{abstract}

\section{Introduction}

We establish class field theory for three-dimensional manifolds and knots in the framework of arithmetic topology. Arithmetic topology is a branch of modern mathematics based on a mysterious analogy between topology and number theory. B. Mazur introduced this area in the study of the Alexander polynomial of a knot in $S^{3}$ in an unpublished note "Remarks on the Alexander polynomial". Morishita also investigated the topic [Mor01, Mor12]. In arithmetic topology, a connected orientable closed three-dimensional manifold is regarded as an analogue of the ring of algebraic integers in a number field, and a knot is regarded as an analogue of a maximal ideal. A branched covering of such manifolds is an analogue of an extension of number fields, and hence class field theory in arithmetic topology means a theory controlling Abelian branched coverings of such manifolds. We note that there are several variants of such correspondences, and hope that readers are not confused about the differences. For example, the ring of algebraic integers in a number field gives two geometric objects that correspond to distinct objects in topology. One is the set of all finite places regarded as an analogue of a non compact 3-manifold, and the other is the set of all places regarded as an analogue of a compact 3-manifold [Den02, $\$ 1, \$ 2$, Appendix].

Our formulation of class field theory in arithmetic topology is a variant of the one originally introduced by $\mathrm{H}$. Niibo and J. Ueki [NU]. They used a homological and cycle-theoretic approach in the formulation of analogues of the multiplicative group and the idèle class group, while we use a cohomological and cocycle-theoretic approach. Although the Poincaré-Lefschetz duality yields several elementary relations between their and our formulations, some results, e.g., descent properties (Theorem 3.8, Theorem 3.10, and Theorem 3.12), the class field axiom (Theorem 3.16), and ideal-theoretic class field theory (Theorem 3.27), essentially rely on the contravariant functoriality of the cocycle-theoretic formulation. Furthermore, we verify several

Received by the editors September 14, 2017; revised March 29, 2018.

Published electronically December 3, 2018.

AMS subject classification: 11Z05, 18F15, 55N20, 57P05.

Keywords: Arithmetic topology, class field theory, branched covering, knots and prime numbers. 
theorems analogous to those in number theory such as Hilbert's Theorem 90 (Theorem 3.7) and the Kronecker-Weber Theorem (Theorem 3.29).

We briefly explain our formulation and results. To begin with, unlike the set of maximal ideals of the ring of algebraic integers in a number field, the set of knots in a connected orientable closed three-dimensional manifold does not make sense in a way compatible with the analogy between an extension of number fields and a branched covering of such manifolds, and one needs to fix a set of knots suitable in a certain aspect. One way to choose such a set was advocated by Niibo and Ueki [NU]. They introduced the notion of a very admissible link as an analogue of the set of maximal ideals and regarded a pair of a connected orientable closed three-dimensional manifold and a very admissible link in it as an analogue of a number field. On the other hand, we introduce the notion of a stably generic link as another analogue of the set of maximal ideals, and regard a pair $K$ of such a manifold $O_{K}$ and a stably generic link $S_{K}$ as an analogue of a number field. A countable stably generic link forms a very admissible link, but the converse does not necessarily hold. Roughly speaking, the definition of a stably generic link is given in a way imitating the assertion of the Chebotarev density theorem. We define a group $K^{\times}$using 1-cocycles of the complements of finite subsets of $S_{K}$, and regard it as an analogue of the multiplicative group of a number field.

For each $\wp \in S_{K}$, we construct a formal limit $K_{\wp}$, i.e., a pro-object, of threedimensional manifolds, and regard it as an analogue of a local field. Since we define an analogue of the multiplicative group in a contravariantly functorial way, the definition naturally extends to a formal limit. We regard the extension $K_{\wp}^{\times}$as an analogue of the multiplicative group of a local field. We construct a local reciprocity map and verify the local existence theorem in Theorem 3.15 through a homomorphism from $K_{\wp}^{\times}$to the singular homology of a torus. Using $\left(K_{\wp}^{\times}\right)_{\wp \in S_{K}}$, we formulate analogues $I_{K}$ and $C_{K}$ of the idèle group and the idèle class group. We construct a global reciprocity map and verify the global existence theorem in Theorem 3.23 through a homomorphism from $C_{K}$ to the projective limit of the singular homologies of open subspaces of $O_{K}$.

We explain the contents of this paper. Section 1 consists of two subsections. In Section 1.1, we introduce several categories of manifolds such as the category of topological manifolds and the category of differentiable manifolds. We also define a category in which Riemannian manifolds naturally lie. In Section 1.2, we introduce formal limits of three-dimensional manifolds, and give examples analogous to local fields and several notions in number theory. Section 2 consists of eleven short subsections. In each subsection, we introduce an analogue of a notion in number theory such as the multiplicative group, the idèle class group, and ray class groups. In particular, we introduce the notion of a stably generic link in Section 2.4. We define $K^{\times}$and $K_{\wp}^{\times}$in Section 2.6, and $I_{K}$ and $C_{K}$ in Section 2.9. We make up the analogues in a table in Section 2.11. Section 3 consists of five subsections. In Section 3.1, we establish Galois theory for branched coverings of three-dimensional manifolds. In Section 3.2, we verify descent property for $K^{\times}, K_{\wp}^{\times}$, and $I_{K}$. In Section 3.3, we verify local class field theory in arithmetic topology. In Section 3.4, we verify global class field theory in arithmetic topology. In Section 3.5, we verify ideal-theoretic class field theory 
in arithmetic topology. In addition, we explain an explicit construction of a stably generic link in Section A.

\section{Preliminaries}

We prepare convention and terminology. We put $\mathbb{N}_{>0}:=\mathbb{N} \backslash\{0\}$. Let $\mathscr{C}$ be a category. We denote by $\mathscr{C}^{\text {op }}$ the opposite category of $\mathscr{C}$, by ob $(\mathscr{C})$ the class of objects in $\mathscr{C}$, by $\operatorname{Hom}_{\mathscr{C}}(X, Y)$ with $(X, Y) \in \mathrm{ob}(\mathscr{C})^{2}$ the set of morphisms $X \rightarrow Y$ in $\mathscr{C}$, by $\operatorname{Aut}_{\mathscr{C}}(X)$ with $X \in \mathrm{ob}(\mathscr{C})$ the automorphism group of $X$ in $\mathscr{C}$, and by $\operatorname{Ind}(\mathscr{C})$ the category of ind-objects in $\mathscr{C}$ and compatible morphisms. Namely, for

$$
\left(X_{j}\right)_{j \in J_{0}} \in \mathrm{ob}(\operatorname{Ind}(\mathscr{C})) \text { and }\left(Y_{j}\right)_{j \in J_{1}} \in \mathrm{ob}(\operatorname{Ind}(\mathscr{C}))
$$

$\operatorname{Hom}_{\operatorname{Ind}(\mathscr{C})}\left(\left(X_{j}\right)_{j \in J_{0}},\left(Y_{j}\right)_{j \in J_{1}}\right)$ is given as $\lim _{j_{0} \in J_{0}} \lim _{j_{1} \in J_{1}} \operatorname{Hom}_{\mathscr{C}}\left(X_{j_{0}}, Y_{j_{1}}\right)$. We have a fully faithful functor $\mathscr{C} \rightarrow \operatorname{Ind}(\mathscr{C})$ sending an object of $\mathscr{C}$ as the ind-object indexed by the singleton $\{\varnothing\}$, through which we identify an object of $\mathscr{C}$ with the image in $\operatorname{Ind}(\mathscr{C})$. We put $\operatorname{Pro}(\mathscr{C}):=\operatorname{Ind}\left(\mathscr{C}^{\text {op }}\right)^{\text {op }}$.

\subsection{Categories of Manifolds}

We introduce several categories related to manifolds on which we work in this paper.

Throughout this paper, a topological space means a Hausdorff space, while a topological group means one which is not necessarily Hausdorff. We denote by Top the category of topological spaces and continuous maps, and by Top ${ }_{0} \subset$ Top the subcategory of local homeomorphisms. For topological spaces $X$ and $Y$, we put $\mathrm{C}(X, Y):=$ $\operatorname{Hom}_{\text {Top }}(X, Y)$. For a topological space $X$, we denote by $\left(\mathrm{H}_{n}(X)\right)_{n \in \mathbb{N}}$ the singular homology of $X$ with coefficients in $\mathbb{Z}$, and by $\left(\mathrm{H}^{n}(X)\right)_{n \in \mathbb{N}}$ the singular cohomology of $X$ with coefficients in $\mathbb{Z}$.

Throughout this paper, a manifold means one with boundary, and a Riemannian manifold means a connected $\mathrm{C}^{\infty}$-manifold with empty boundary equipped with a fixed Riemannian metric. For a $\kappa \in \mathbb{N} \sqcup\{\infty\}$, we denote by $\mathrm{C}^{\kappa}$ the category of $\mathrm{C}^{\kappa}$-manifolds and maps of class $\mathrm{C}^{\kappa}$, and by $\mathrm{C}_{0}^{\kappa} \subset \mathrm{C}^{\kappa}$ the subcategory of local isomorphisms.

We denote by Met the category of metric spaces and locally Lipschitz maps, and by $\mathrm{Met}^{\infty}$ the category of Riemannian manifolds and maps of class $\mathrm{C}^{\infty}$. We have a natural functor $\mathscr{F}^{\text {Met: }}$ : Met ${ }^{\infty} \rightarrow$ Met, because every map of class $\mathrm{C}^{\infty}$ between Riemannian manifolds is locally Lipschitz with respect to the Riemannian metrics. We also define another category containing branched coverings of Riemannian manifolds.

For a topological space $X$ and a $\left(\gamma_{0}, \gamma_{1}\right) \in \mathrm{C}([0,1], X)^{2}$ with $\gamma_{0}(1)=\gamma_{1}(0)$, we define $\gamma_{0} * \gamma_{1} \in \mathrm{C}([0,1], X)$ by setting $\left(\gamma_{0} * \gamma_{1}\right)(t):=\gamma_{0}(2 t)$ for any $t \in\left[0,2^{-1}\right]$ and $\left(\gamma_{0} * \gamma_{1}\right)(t):=\gamma_{1}(2 t-1)$ for any $t \in\left(2^{-1}, 1\right]$. A path-length space is a pair $(X, \ell)$ of a topological space $X$ and a map $\ell: C([0,1], X) \rightarrow[0, \infty]$ satisfying the following.

- For any $\gamma \in \mathrm{C}([0,1], X)$, if $\gamma$ is a constant map, then $\ell(\gamma)=0$.

- If $(\gamma, g) \in \mathrm{C}([0,1], X) \times \operatorname{Aut}_{\mathrm{Top}}([0,1])$, then $\ell(\gamma \circ g)=\ell(\gamma)$.

- If $\left(\gamma_{0}, \gamma_{1}\right) \in \mathrm{C}([0,1], X)^{2}$ with $\gamma_{0}(1)=\gamma_{1}(0)$, then $\ell\left(\gamma_{0} * \gamma_{1}\right)=\ell\left(\gamma_{0}\right)+\ell\left(\gamma_{1}\right)$.

We give two examples of path-length spaces. 
Example 1.1 Let $(\mathscr{X}, d)$ be a metric space. We denote by $X$ the underlying topological space of $(\mathscr{X}, d)$. For an $n \in \mathbb{N}$, we denote by $\Delta_{n} \subset[0,1]^{n}$ the subset of increasing sequences. For a $\gamma \in \mathrm{C}([0,1], X)$, we denote by $\ell_{\gamma} \in[0, \infty]$ the path-length $\sup _{n \in \mathbb{N}} \sup _{\left(t_{i}\right)_{i=1}^{n} \in \Delta_{n+1}} \sum_{i=0}^{n-1} d\left(\gamma\left(t_{i}\right), \gamma\left(t_{i+1}\right)\right)$ of $\gamma$. Then the pair $\left(X, \ell_{d}\right)$ of $X$ and the map $\ell_{d}: \mathrm{C}([0,1], X) \rightarrow[0, \infty], \gamma \mapsto \ell_{\gamma}$ forms a path-length space. We always regard a metric space as a path-length space in this way.

Example 1.2 Let $(S, \ell)$ be a path-length space, and $X$ a topological space equipped with a $\varphi \in \mathrm{C}(X, S)$. Then the pair $\left(X, \varphi^{*}(\ell)\right)$ of $X$ and the map

$$
\varphi^{*}(\ell): \mathrm{C}([0,1], X) \longrightarrow[0, \infty], \gamma \longmapsto \ell(\varphi \circ \gamma)
$$

form a path-length space.

Every Riemannian manifold forms a metric space, and hence forms a path-length space by Example 1.1. Therefore, every branched covering of a Riemannian manifold naturally forms a path-length space by Example 1.2. The notion of a path-length space is interesting in class field theory in arithmetic topology, because one can formulate the length of a knot as an analogue of the cardinality of the residue field at a maximal ideal (Remark 2.7).

We introduce several specific types of morphisms between path-length spaces. Let $\left(X, \ell_{X}\right)$ and $\left(Y, \ell_{Y}\right)$ be path-length spaces. A strict morphism $\left(X, \ell_{X}\right) \rightarrow\left(Y, \ell_{Y}\right)$ is a continuous map $\varphi: X \rightarrow Y$ with $\varphi^{*}\left(\ell_{Y}\right)=\ell_{X}$. A local isomorphism $\left(X, \ell_{X}\right) \rightarrow\left(Y, \ell_{Y}\right)$ is a strict morphism $\left(X, \ell_{X}\right) \rightarrow\left(Y, \ell_{Y}\right)$ that is also a local homeomorphism $X \rightarrow Y$. A Lipschitz morphism $\left(X, \ell_{X}\right) \rightarrow\left(Y, \ell_{Y}\right)$ is a continuous map $\varphi: X \rightarrow Y$ such that there exists a $C \in(0, \infty)$ with $\ell_{Y}(\varphi \circ \gamma) \leq C \ell_{X}(\gamma)$ for any $\gamma \in \mathrm{C}([0,1], X)$. A locally Lipschitz morphism $\left(X, \ell_{X}\right) \rightarrow\left(Y, \ell_{Y}\right)$ is a continuous map $\varphi: X \rightarrow Y$ such that there exists an open covering $\mathscr{U}$ of $X$ such that for any $U \in \mathscr{U},\left.\varphi\right|_{U}$ forms a Lipschitz morphism $\left(U, i_{U}^{*}\left(\ell_{X}\right)\right) \rightarrow\left(Y, \ell_{Y}\right)$, where $i_{U}$ denotes the inclusion $U \rightarrow X$. Every strict morphism is a Lipschitz morphism, and every Lipschitz morphism is a locally Lipschitz morphism. The composite of strict morphisms (resp. local isomorphisms, Lipschitz morphisms, locally Lipschitz morphisms) is again a strict morphism (resp. local isomorphism, Lipschitz morphism, locally Lipschitz morphism).

We denote by PLSp the category of path-length spaces and locally Lipschitz morphisms, and by PLSp $p_{0} \subset \mathrm{PLSp}$ the full subcategory of local isomorphisms. We have a natural functor Met $\rightarrow$ PLSp because every locally Lipschitz map between metric spaces forms a locally Lipschitz morphism between the associated path-length spaces.

\subsection{Categories of Pro-manifolds}

Henceforth, we denote by $\mathscr{C}$ one of the categories Top ${ }^{\text {op }},\left(C^{\kappa}\right)^{\text {op }}$ with $\kappa \in \mathbb{N} \sqcup\{\infty\}$, and PLSp ${ }^{\text {op }}$. We put $\mathscr{C}_{0}:=\operatorname{Top}_{0}^{\text {op }}$ when $\mathscr{C}=\operatorname{Top}^{\text {op }}, \mathscr{C}_{0}:=\left(\mathrm{C}_{0}^{\kappa}\right)^{\text {op }}$ when $\mathscr{C}=\left(\mathrm{C}^{\kappa}\right)^{\text {op }}$, and $\mathscr{C}_{0}:=\mathrm{PLSp}_{0}^{\text {op }}$ when $\mathscr{C}=\mathrm{PLSp}^{\text {op }}$. We denote by $\mathscr{F}$ the forgetful contravariant functor $\mathscr{C} \rightarrow$ Top, and by $\mathscr{F}_{0}$ the forgetful contravariant functor $\mathscr{C}_{0} \rightarrow$ Top $_{0}$.

Let $O \in \mathrm{ob}\left(\mathscr{C}_{0}\right)$. For a topological space $U$ equipped with a local homeomorphism $\varphi: U \rightarrow \mathscr{F}_{0}(O)$, we denote by $\left.O\right|_{U}$ the object of $\mathscr{C}_{0}$ with $\mathscr{F}_{0}\left(\left.O\right|_{U}\right)=U$ such that $\varphi$ 
gives a morphism $\left.O\right|_{U} \rightarrow O$ in $\mathscr{C}_{0}$. The unique existence of such an object is obvious when $\mathscr{C}=$ Top $^{\text {op }}$ or $\mathscr{C}=\left(\mathrm{C}^{\kappa}\right)^{\text {op }}$, and follows from the construction in Example 1.2 when $\mathscr{C}=\mathrm{PLSp}^{\text {op }}$. For a finite set $s$ of closed subsets of $\mathscr{F}_{0}(O)$, we put $O\left[s^{-1}\right]:=$ $\left.O\right|_{\mathscr{F}_{0}(O) \backslash \cup_{\wp \in s} \wp} \in \mathrm{ob}\left(\mathscr{C}_{0}\right)$.

We abbreviate Ind $(\mathscr{C})$ to $\widehat{\mathscr{C}}$ and $\operatorname{Ind}\left(\mathscr{C}_{0}\right)$ to $\widehat{\mathscr{C}}_{0}$. We give several examples of objects of $\widehat{\mathscr{C}}_{0}$, all of which play important roles in this paper.

Example 1.3 (localisation) Let $S$ be a set of pairwise disjoint closed subsets of $\mathscr{F}_{0}(O)$. We denote by $\operatorname{Fin}(S)$ the set of finite subsets of $S$ directed by the order $\leq$ given by setting $s_{0} \leq s_{1}$ if and only if $s_{0} \subset s_{1}$. Then $\left(O\left[s^{-1}\right]\right)_{s \in \operatorname{Fin}(s)}$ forms an object $S^{-1} O$ of $\widehat{\mathscr{C}}_{0}$ with respect to the compatible system of morphisms contravariantly corresponding to the inclusions, and plays a role analogous to the localisation $\Sigma^{-1} \mathscr{O}$ of a ring $\mathscr{O}$ by a multiplicative subset $\Sigma$. When $S$ is a finite set, then $S^{-1} O \in \widehat{\mathscr{C}}_{0}$ is naturally isomorphic to the image of $O\left[S^{-1}\right] \in \mathrm{ob}\left(\mathscr{C}_{0}\right)$ in $\widehat{\mathscr{C}}_{0}$, because $S$ is the greatest element of $\operatorname{Fin}(S)$.

Example 1.4 (completion) Let $\wp$ be a subset of $\mathscr{F}_{0}(O)$. We denote by $\operatorname{Nbh}(O, \wp)$ the set of open neighbourhoods of $\wp$ in $\mathscr{F}_{0}(O)$, which is directed by the order $\leq$ given by setting $U_{0} \leq U_{1}$ if and only if $U_{1} \subset U_{0}$. Then $\left(\left.O\right|_{U}\right)_{U \in \operatorname{Nbh}(O, \wp)}$ forms an object $O_{\wp}$ of $\widehat{\mathscr{C}}_{0}$ with respect to the compatible system of morphisms contravariantly corresponding to the inclusions, and plays roles analogous to the Henselisation of a ring $\mathscr{O}$ at a prime ideal $P$ and to the completion $\mathscr{O}_{P}$ of $\mathscr{O}$ at $P$. When $\wp$ is closed, then $\left(\left.O\right|_{U \backslash \wp}\right)_{U \in \operatorname{Nbh}(O, \wp)}$ forms an object $\operatorname{Frac}\left(O_{\wp}\right)$ of $\widehat{\mathscr{C}}_{0}$ in a similar way, and plays a role analogous to the total ring $\operatorname{Frac}\left(\mathscr{O}_{P}\right)$ of fractions of $\mathscr{O}_{P}$.

Example 1.5 (unramified extension) Let $\iota_{\infty} \in \mathscr{F}_{0}(O)$. Suppose that $\mathscr{F}_{0}(O)$ is a path-connected locally path-connected semi-locally simply connected topological space. We denote by $\left(O, \iota_{\infty}\right)^{\sim}$ the universal covering of $\mathscr{F}_{0}(O)$ given as the set of homotopy classes of continuous maps $\gamma:[0,1] \rightarrow \mathscr{F}_{0}(O)$ with $\gamma(0)=\iota_{\infty}$, and by $\operatorname{Cov}\left(O, \iota_{\infty}\right)$, the set of finite unbranched coverings of $\mathscr{F}_{0}(O)$ given as quotients of $\left(O, \iota_{\infty}\right)^{\sim}$, which is directed by the order $\leq$ given by setting $N_{0} \leq N_{1}$ if and only if the canonical projection $N_{1} \rightarrow \mathscr{F}_{0}(O)$ factors through the canonical projection $N_{0} \rightarrow$ $\mathscr{F}_{0}(O)$. Then $\left(\left.O\right|_{N}\right)_{N \in \operatorname{Cov}\left(O, \iota_{\infty}\right)}$ forms an object $\left(O, \iota_{\infty}\right)$ ur of $\widehat{\mathscr{C}}_{0}$ with respect to the canonical projections, and plays a role analogous to the integral closure $\left(\mathscr{O}, i_{\infty}\right)$ ur of a ring $\mathscr{O}$ of algebraic numbers in the maximal unramified extension $\left(\operatorname{Frac}(\mathscr{O}), i_{\infty}\right){ }^{\mathrm{ur}} \subset$ $\mathbb{C}$ of the fractional field $\operatorname{Frac}(\mathscr{O})$ with respect to a fixed embedding $i_{\infty}: \operatorname{Frac}(\mathscr{O}) \hookrightarrow \mathbb{C}$.

A $\mathscr{C}$-manifold means a topological manifold when $\mathscr{C}=\mathrm{Top}^{\mathrm{op}}$, a $\mathrm{C}^{\kappa}$-manifold when $\mathscr{C}=\left(\mathrm{C}^{\mathcal{K}}\right)^{\text {op }}$, and a path-length space whose underlying topological space is a topological manifold when $\mathscr{C}=\mathrm{PLSp}^{\text {op }}$. A strict $\mathscr{C}$-manifold means a connected $\mathrm{C}^{\infty}$-manifold when $\mathscr{C}=\mathrm{Top}^{\text {op }}$ or $\mathscr{C}=\left(\mathrm{C}^{\kappa}\right)^{\text {op }}$, and a Riemannian manifold when $\mathscr{C}=$ PLSp $^{\text {op }}$. Every Riemannian manifold is a strict $\mathscr{C}$-manifold, every strict $\mathscr{C}$-manifold is a $\mathscr{C}$-manifold, and every $\mathscr{C}$-manifold $X$ naturally forms an object $\mathscr{A}_{X}$ of $\mathscr{C}_{0}$.

A $\mathscr{C}$-morphism between $\mathscr{C}$-manifolds means a continuous map when $\mathscr{C}=$ Top $^{\text {op }}$, a map of class $\mathrm{C}^{\kappa}$ when $\mathscr{C}=\left(\mathrm{C}^{\mathcal{k}}\right)^{\text {op }}$, and a locally Lipschitz map when $\mathscr{C}=$ PLSp $^{\text {op }}$. 
The composite of $\mathscr{C}$-morphisms again forms a $\mathscr{C}$-morphism. Every map of class $\mathrm{C}^{\infty}$ between strict $\mathscr{C}$-manifolds is a $\mathscr{C}$-morphism. Every $\mathscr{C}$-morphism $\varphi: X \rightarrow Y$ gives a morphism $\mathscr{A}_{\varphi}: \mathscr{A}_{Y} \rightarrow \mathscr{A}_{X}$ in $\mathscr{C}$. We denote by Mfd the category of $\mathscr{C}$-manifolds and $\mathscr{C}$-morphisms. The correspondence $X \leadsto \mathscr{A}_{X}$ gives a contravariant functor Mfd $\rightarrow \mathscr{C}$.

Let $r \in(0,1]$ and $n \in \mathbb{N}$. All of $S^{1}=\{z \in \mathbb{C}|| z \mid=1\}, \mathrm{D}^{2}[r):=\{w \in \mathbb{C}|| w \mid<r\}$, $\mathrm{D}^{2}(r):=\{w \in \mathbb{C}|0<| w \mid<r\}, V[r):=\mathrm{S}^{1} \times \mathrm{D}^{2}[r), V(r):=\mathrm{S}^{1} \times \mathrm{D}^{2}(r)$, and $\Delta^{n}=\left\{\left(t_{i}\right)_{i=0}^{n} \in[0,1]^{n+1} \mid \sum_{i=0}^{n} t_{i}=1\right\}$ are both $C^{\infty}$-manifolds and metric spaces, and hence naturally form $\mathscr{C}$-manifolds. We put $\mathbb{S}^{1}:=\left(\mathscr{A}_{V[1)}\right)_{S^{1} \times\{0\}} \in \operatorname{ob}\left(\widehat{\mathscr{C}}_{0}\right)$ and $\mathbb{T}^{2}:=\operatorname{Frac}\left(\left(\mathscr{A}_{V[1)}\right)_{\mathrm{S}^{1} \times\{0\}}\right) \in \mathrm{ob}\left(\widehat{\mathscr{C}}_{0}\right)$ (Example 1.4). Then $\mathbb{S}^{1}$ (resp. $\mathbb{T}^{2}$ ) is naturally isomorphic to $\left(\mathscr{A}_{V[r)}\right)_{r \in(0,1]}$ (resp. $\left.\left(\mathscr{A}_{V(r)}\right)_{r \in(0,1)]}\right)$ in $\widehat{\mathscr{C}}_{0}$ because $\{V[r) \mid r \in(0,1)\}$ is cofinal in $\mathrm{Nbh}\left(V[1), S^{1} \times\{0\}\right)$. The basic objects $\mathscr{A}_{\mathrm{S}^{1}}, \mathbb{S}^{1}$, and $\mathbb{T}^{2}$ play roles analogous to $\mathbb{F}_{q}, \mathbb{Z}_{q}$, and $\mathbb{Q}_{q}$, respectively, where $q$ is a power of a prime number.

\section{$2 M^{2}$ KR Dictionary}

We introduce analogues of several notions appearing in class field theory in number theory, and give a variant of $\mathrm{M}^{2} \mathrm{KR}$ dictionary admissible to our cohomological approach to class field theory in arithmetic topology. We recall that an $\mathrm{M}^{2} \mathrm{KR}$ dictionary means a table on the analogy between low-dimensional topology and number theory, and is named after the originators of arithmetic topology: B. Mazur, M. Morishita, M. M. Kaplanov, and A. Reznikov. In order to help readers working on number theory to grasp the analogy well, we use symbols imitating the notation in number theory. The reason why the ones introduced can be seen as analogues will become clear in Section 3 through the formulation of class field theory in arithmetic topology.

\subsection{Analogue of a Maximal Ideal}

A morphism in $\mathscr{C}$ is said to be a local isomorphism in $\mathscr{C}$ if it is given as the composite of a morphism in $\mathscr{C}_{0}$ followed by an isomorphism in $\mathscr{C}$. Since a pullback in $\mathscr{C}$ of any morphism in $\mathscr{C}$ by any morphism in $\mathscr{C}_{0}$ exists, the composite of an isomorphism in $\mathscr{C}$ followed by a local isomorphism in $\mathscr{C}$ is again a local isomorphism in $\mathscr{C}$. In particular, the composite of local isomorphisms in $\mathscr{C}$ is again a local isomorphism in $\mathscr{C}$.

Let $O \in \mathrm{ob}\left(\mathscr{C}_{0}\right)$. For an $r \in(0,1]$ and a $j \in \operatorname{Hom}_{\mathscr{C}}\left(O, \mathscr{A}_{V[r)}\right)$, we denote by $j_{S^{1}} \in \operatorname{Hom}_{\mathscr{C}}\left(O, \mathscr{A}_{S^{1}}\right)$ the composite of $j$ and the morphism $\mathscr{A}_{V[r)} \rightarrow \mathscr{A}_{S^{1}}$ in $\mathscr{C}_{0}$ given as the zero embedding $S^{1} \hookrightarrow V[r), z \mapsto(z, 0)$.

Let $\chi \in \operatorname{Hom}_{\mathscr{C}}\left(O, \mathscr{A}_{\mathrm{S}^{1}}\right)$. We put $\operatorname{im}(\chi):=\operatorname{im}(\mathscr{F}(\chi)) \subset \mathscr{F}_{0}(O)$. A

$$
j \in \operatorname{Hom}_{\mathscr{C}}\left(O, \mathscr{A}_{V[r)}\right)
$$

with $r \in(0,1]$ is said to be a tame extension of $\chi$ if $j$ is a local isomorphism with $j_{\mathrm{S}^{1}}=\chi$. We say that $\chi$ is a cycle in $O$ if $\mathscr{F}(\chi): S^{1} \rightarrow \mathscr{F}_{0}(O)$ is injective. A cycle in $O$ is said to be tame if it admits a tame extension. The notion of a tame cycle is an analogue of a homomorphism from a ring $\mathscr{O}$ of algebraic integers onto $\mathbb{F}_{q}$ with a power $q$ of a prime number.

We denote by $\operatorname{Aut}_{\mathscr{C}}^{+}\left(\mathscr{A}_{\mathrm{S}^{1}}\right) \subset \operatorname{Aut}_{\mathscr{C}}\left(\mathscr{A}_{\mathrm{S}^{1}}\right)$ the subgroup consisting of automorphisms $\sigma$ such that $\mathscr{F}(\sigma)$ acts trivially on $\mathrm{H}_{1}\left(\mathrm{~S}^{1}\right)$. The set of cycles in $O$ and the set of tame cycles in $O$ are stable under the action of $\operatorname{Aut}_{\mathscr{C}}\left(\mathscr{A}_{\mathrm{S}^{1}}\right)$ on $\operatorname{Hom}_{\mathscr{C}}\left(O, \mathscr{A}_{\mathrm{S}^{1}}\right)$ 
given by the composition from the right. A knot in $O$ is an equivalence class of cycles in $O$ with respect to the right action of $\operatorname{Aut}_{\mathscr{C}}^{+}\left(\mathscr{A}_{\mathrm{S}^{1}}\right)$. A knot in $O$ is said to be tame if it consists of tame cycles in $O$. The notion of a tame knot is an analogue of a maximal ideal $P$ of $\mathscr{O}$, which is a generalisation of the ideal generated by a prime number.

Let $\wp$ be a knot in $O$. A $j \in \operatorname{Hom}_{\mathscr{C}}\left(O, \mathscr{A}_{V[r)}\right)$ with $r \in(0,1]$ is said to be a tubular neighbourhood of $\wp$ if $j_{\mathcal{S}^{1}}$ is a cycle in $O$ representing $\wp$. By definition, $\wp$ is tame if and only if $\wp$ admits a tubular neighbourhood. Moreover, if there is an orientable strict $\mathscr{C}$-manifold $X$ with $\mathscr{A}_{X}=O$, then $\wp$ is tame if and only if there is a map $S^{1} \rightarrow X$ of class $\mathrm{C}^{\infty}$ representing $\wp$ by tubular neighbourhood theorem [Hir76, Theorem 4.5.2].

We take a representative $\chi \in \operatorname{Hom}_{\mathscr{C}}\left(O, \mathscr{A}_{\mathrm{S}^{1}}\right)$ of $\wp$, and put $\operatorname{im}(\wp):=\operatorname{im}(\chi)$. We denote by $E$ the surjective continuous map $\mathbb{R} \rightarrow S^{1}, t \mapsto \exp (2 \pi \sqrt{-1} t)$, by $\theta$ the surjective continuous map $\Delta^{1} \rightarrow S^{1},\left(t_{0}, t_{1}\right) \mapsto E\left(t_{1}\right)$, and by $\operatorname{Frob}_{\wp} \in \mathrm{H}_{1}\left(\mathscr{F}_{0}(O)\right)$ the homology class of the singular 1-cycle represented by the continuous map

$$
\mathscr{F}(\chi) \circ \theta: \Delta^{1} \rightarrow \mathscr{F}_{0}(O) .
$$

We also denote by Frob $\wp_{\wp}$ the images of Frob ${ }_{\wp}$ by natural group homomorphisms as long as there is no ambiguity. Then $\operatorname{im}(\wp)$ and Frob $\wp$ are independent of the choice of $\chi$ by the definition of the action of $\operatorname{Aut}_{\mathscr{C}}^{+}\left(\mathscr{A}_{\mathrm{S}^{1}}\right)$. They play roles analogous to the finite field $\mathscr{O} / P$ and the Frobenius automorphism at $P$ of a maximal unramified Abelian extension of $\operatorname{Frac}(\mathscr{O})$, respectively. We call $\operatorname{Frob}_{\wp}$ the Frobenius of $O$ at $\wp$.

Suppose $\mathscr{C}=\mathrm{PLSp}^{\mathrm{op}}$. We put $O=\left(M, \ell_{M}\right)$. By the definition of a path-length, $\ell_{O}(\wp):=\ell_{M}(\chi)$ is independent of the choice of $\chi \in \wp$. We put $\# O / \wp:=\exp \left(\ell_{O}(\wp)\right)$, and call it the exponential length of $O / \wp$. Then $\# O / \wp$ is analogous to the cardinality of $\mathscr{O} / P$.

Since $\chi$ is a continuous map from a compact topological space to a Hausdorff topological space, $\operatorname{im}(\wp)$ is closed. Therefore we obtain the objects

$$
O_{\operatorname{im}(\wp)} \text { and } \operatorname{Frac}\left(O_{\operatorname{im}(\wp)}\right)
$$

of $\widehat{\mathscr{C}}_{0}$ (Example 1.4). We abbreviate $O_{\mathrm{im}(\wp)}$ to $O_{\wp}$ and $\operatorname{Frac}\left(O_{\mathrm{im}(\wp)}\right)$ to $\operatorname{Frac}\left(O_{\wp}\right)$. We have canonical morphisms $O \rightarrow O_{\wp}$ and $O_{\wp} \rightarrow \operatorname{Frac}\left(O_{\wp}\right)$ in $\widehat{\mathscr{C}}_{0}$ given by the compatible systems of restrictions of $\operatorname{id}_{\mathscr{F}_{0}(O)}$.

\subsection{Partial Analogue of a Number Field}

Let $O \in \mathrm{ob}\left(\mathscr{C}_{0}\right)$. We say that $O$ is pre-arithmetic (resp. arithmetic) if there is a connected three-dimensional $\mathscr{C}$-manifold (resp. a connected three-dimensional strict $\mathscr{C}$ manifold) $X$ with $\partial X=\varnothing$ and $\mathscr{A}_{X}=O$. We note that the difference between the notions of a pre-arithmetic object and an arithmetic object of $\mathscr{C}_{0}$ occurs only when $\mathscr{C}=$ PLSp ${ }^{\text {op }}$, because every three-dimensional topological manifold (resp. three-dimensional $\mathrm{C}^{\kappa}$-manifold) admits a compatible structure of a $\mathrm{C}^{\infty}$-manifold.

Suppose that $O$ is pre-arithmetic. We say that $O$ is closed (resp. orientable) if such an $X$ can be taken as a closed (resp. orientable strict) $\mathscr{C}$-manifold. The notion of an orientable closed arithmetic object of $\mathscr{C}_{0}$ is analogous to a ring $\mathscr{O}$ of algebraic integers. While a number field is equipped with the set of maximal ideals, an orientable closed arithmetic object of $\mathscr{C}_{0}$ is not naturally equipped with a set of knots. Therefore we need to consider a counterpart of the set of maximal ideals. 
Let $S$ be a set of knots in $O$. We put $\operatorname{im}(S):=\bigcup_{\wp \in S} \operatorname{im}(\wp) \subset \mathscr{F}_{0}(O)$. We say that $S$ is a link in $O$ if $\operatorname{im}(S) \neq \mathscr{F}_{0}(O)$ and $\operatorname{im}\left(\wp_{0}\right) \cap \operatorname{im}\left(\wp_{1}\right)=\varnothing$ for any $\left(\wp_{0}, \wp_{1}\right) \in S^{2}$ with $\wp_{0} \neq \wp_{1}$. The condition $\operatorname{im}(S) \neq \mathscr{F}_{0}(O)$ automatically holds when $S$ is countable by the Baire category theorem. Suppose that $S$ is a link in $O$. Then $\{\operatorname{im}(\wp) \mid \wp \in S\}$ is a set of pairwise disjoint closed subsets of $O$. We abbreviate $\{\operatorname{im}(\wp) \mid \wp \in S\}^{-1} O \in \operatorname{ob}\left(\widehat{\mathscr{C}}_{0}\right)$ (Example 1.3) to $S^{-1} O$. When $S$ is finite, we abbreviate

$$
O\left[\{\operatorname{im}(\wp) \mid \wp \in S\}^{-1}\right] \in \mathrm{ob}\left(\mathscr{C}_{0}\right)
$$

to $O\left[S^{-1}\right]$.

We say that $S$ is tame if $S$ consists of tame knots, is admissible if $S$ is tame and $\left\{\right.$ Frob $\left._{\wp} \mid \wp \in S\right\}$ generates $\mathrm{H}_{1}\left(\mathscr{F}_{0}(O)\right)$, and is generic if $S \backslash s$ forms an admissible link in $O\left[s^{-1}\right]$ for any $s \in \operatorname{Fin}(S)$. If $S$ is generic, then $S$ is admissible. The definitions of an admissible link and a generic link are analogous to the Chebotarev density theorem. The notion of a generic link is analogous to the set $\operatorname{Max}(\mathscr{O})$ of maximal ideals of $\mathscr{O}$, which is a generalisation of the set of prime numbers.

An arithmetic manifold is a pair $K=\left(O_{k}, S_{K}\right)$ of a pre-arithmetic object $O_{K}$ of $\mathscr{C}_{0}$ and a tame link $S_{K}$ in $O_{K}$ admitting an $s \in \operatorname{Fin}\left(S_{K}\right)$ such that $O_{K}\left[s^{-1}\right]$ is arithmetic. Let $K$ be an arithmetic manifold. We say that $K$ is closed if $O_{K}$ is closed, is orientable if there is an $s \in \operatorname{Fin}\left(S_{K}\right)$ such that $O_{K}\left[s^{-1}\right]$ is orientable, and is admissible (resp. generic) if $S_{K}$ is admissible (resp. generic).

The notion of an orientable closed generic arithmetic manifold is partially analogous to a number field. We will define the notion of a covering of an arithmetic manifold in Section 2.3, and it would be natural to regard the notion of a covering of an orientable closed generic arithmetic manifold as an analogue of a finite extension of a number field, which is again a number field. However, a covering of an orientable closed generic arithmetic manifold forms an orientable closed arithmetic manifold that is not necessarily generic. For this reason, we will introduce a condition stably generic which is stronger than generic in Section 2.4.

For a $\wp \in K$, we abbreviate $\operatorname{Frac}\left(\left(O_{K}\right)_{\wp}\right)$ (Example 1.4) to $K_{\wp}$, and call it the formal completion of $K$ at $\wp$. As a special case of Example 1.4, $K_{\wp}$ is partially analogous to the Henselisation and the completion $k_{p}$ of a number field $k$ at a maximal ideal $p$ of the ring of algebraic integers in $k$.

\subsection{Partial Analogue of an Extension of Number Fields}

Continuing from Section 2.2, let $K=\left(O_{K}, S_{K}\right)$ denote an arithmetic manifold. We put $M:=\mathscr{F}_{0}\left(O_{K}\right)$. We give a partial analogue of an extension of number fields. Throughout this paper, we follow the terminology on branched coverings [Fox57, p. 250]. We note that since $K$ is an arithmetic manifold, $M$ is a connected locally compact topological space that is always assumed to be Hausdorff, and hence satisfies the assumption of the covering space and the base space of a branched covering [Fox57].

A topological covering of $K$ is a pair $(N, h)$ of a connected locally compact topological space $N$ and a continuous map $h: N \rightarrow M$ such that $h$ is a branched covering that is unbranched of finite degree outside $\operatorname{im}(s)$ for some $s \in \operatorname{Fin}\left(S_{K}\right)$. For topological coverings $\left(N_{0}, h_{0}\right)$ and $\left(N_{1}, h_{1}\right)$ of $K$, a covering morphism $\left(N_{1}, h_{1}\right) \rightarrow\left(N_{0}, h_{0}\right)$ over 
$K$ is a continuous map $\varphi: N_{1} \rightarrow N_{0}$ with $h_{0} \circ \varphi=h_{1}$. We denote by Top / $K$ the category of topological coverings of $K$ and covering morphisms over $K$.

We give a construction of an arithmetic manifold from a topological covering of $K$. For this purpose, we introduce a convention on a branched covering of tori. Let $r \in(0,1]$. For a $(d, e, f) \in \mathbb{Z} \times \mathbb{N}_{>0} \times \mathbb{N}_{>0}$, we denote by $h_{d, e, f}[r)$ the branched covering $V\left[r^{e^{-1}}\right) \rightarrow V[r),(z, w) \mapsto\left(z^{f}, z^{d} w^{e}\right)$ unbranched outside $S^{1} \times\{0\}$ of degree $e f$, and call it $a$ standard branched covering. For any branched covering $h: V \rightarrow V[r)$ unbranched outside $S^{1} \times\{0\}$ of finite degree, the subgroup of $\mathrm{H}_{1}(V(r)) \cong \mathbb{Z}^{2}$ corresponding to the unbranched covering associated with $h$ through the Hurewicz isomorphism $\mathrm{H}_{1}(V(r)) \cong \pi_{1}\left(V(r),\left(1,2^{-1} r\right)\right)^{\mathrm{ab}} \cong \pi_{1}\left(V(r),\left(1,2^{-1} r\right)\right)$ is generated by the homology classes of the singular 1-cycles represented by the continuous maps $\left(\theta^{f}, 2^{-1} r\right)$ and $\left(\theta^{d}, 2^{-1} r \theta^{e}\right)$ for a unique $(d, e, f) \in \mathbb{Z} \times \mathbb{N}_{>0} \times \mathbb{N}_{>0}$, and there is a homeomorphism $\varphi: V \rightarrow V\left[r^{e^{-1}}\right)$ with $h_{d, e, f}[r) \circ \varphi=h$.

Let $(N, h)$ be a topological covering of $K$. When $\mathscr{C}=$ Top $^{\text {op }}$, we put

$$
h^{*}\left(O_{K}\right):=N \in \mathrm{ob}(\mathrm{Top}) \text {. }
$$

When $\mathscr{C}=\mathrm{PLSp}^{\mathrm{op}}, O_{K}$ is presented as a pair $(M, \ell)$, and we put

$$
h^{*}\left(O_{K}\right):=\left(N, h^{*} \ell\right) \in \mathrm{ob}(\mathrm{PLSp}) \quad \text { (Example 1.2). }
$$

Suppose $\mathscr{C}=\left(\mathrm{C}^{\kappa}\right)^{\text {op }}$. We denote by $U \subset N$ the open subset given as the antecedent of the unbranched covering associated with $h$, which forms a $\mathrm{C}^{\kappa}$-manifold with respect to the pullback of the $\mathrm{C}^{\kappa}$-manifold structure of $O_{K}$. For each $\wp \in S_{K}$, we take a tubular neighbourhood $j_{\wp} \in \operatorname{Hom}_{\mathscr{C}}\left(O_{K}, \mathscr{A}_{V\left[r_{\wp}\right)}\right)$ with $r_{\wp} \in(0,1]$ of $\wp$. Replacing $\left(r_{\wp}\right)_{\wp \in S_{K}}$ by a family of smaller ones, we may assume that for any $\wp \in S_{K}$, $h$ is unbranched over $\mathscr{F}\left(j_{\wp}\right)\left(V\left(r_{\wp}\right)\right)$ and hence the pullback of $h$ by $j_{\wp}$ in Top is given as $h_{d, e, f}\left[r_{\wp}\right)$ for a $(d, e, f) \in \mathbb{Z} \times \mathbb{N}_{>0} \times \mathbb{N}_{>0}$. It implies that there is a surjective local homeomorphism $\varphi: U \sqcup \bigsqcup_{i=1}^{m} V\left[r_{i}\right) \rightarrow N$ with $m \in \mathbb{N}$ and $\left(r_{i}\right)_{i=1}^{m} \in(0,1]^{m}$ for which $h \circ \varphi: U \sqcup \bigsqcup_{i=1}^{m} V\left[r_{i}\right) \rightarrow O_{K}$ is of class $C^{\kappa}$. We equip $N$ with a unique structure of a $\mathrm{C}^{\kappa}$-manifold for which $\varphi$ is of class $\mathrm{C}^{\kappa}$, and denote by $h^{*}\left(O_{K}\right)$ the resulting $\mathrm{C}^{\kappa}$-manifold. It is obvious that $h^{*}\left(O_{K}\right)$ depends only on $(N, h)$ and is a connected threedimensional $\mathscr{C}$-manifold with $\mathscr{F}_{0}\left(h^{*}\left(O_{K}\right)\right)=N$ and $h \in \operatorname{Hom}_{\mathscr{C}_{0}}\left(h^{*}\left(O_{K}\right), O_{K}\right)$. If $O_{K}$ is a closed (resp. an orientable strict) $\mathscr{C}$-manifold, then so is $h^{*}\left(O_{K}\right)$ by the construction.

Remark 2.1 When $h$ is an unbranched covering, the structure of $h^{*}\left(O_{K}\right)$ is just given as the pullback of the structure of $O_{K}$, and hence $h$ regarded as a morphism $h^{*}\left(O_{K}\right) \rightarrow O_{K}$ in $\mathscr{C}_{0}$ satisfies the covering homotopy property with respect to any $\mathscr{C}$-morphism whose target is of the form $\mathscr{A}_{M}$ for some compact $\mathscr{C}$-manifold $M$ admitting a $\mathscr{C}$-morphism $M \times[0,1] \rightarrow M$ that is a homotopy connecting $\mathrm{id}_{M}$ and a constant map $M \rightarrow M$.

We denote by $h^{*}\left(S_{K}\right)$ the set of knots $\mathscr{P}$ in $h^{*}\left(O_{K}\right)$ for which there is an

$$
(f, \wp) \in \mathbb{N}_{>0} \times S_{K}
$$

such that for any $\tilde{\chi} \in \mathscr{P}, h \circ \widetilde{\chi}$ is presented as the map $S^{1} \rightarrow O_{K}, z \mapsto \chi\left(z^{f}\right)$ for some $\chi \in \wp$. Such a $\wp$ is obviously unique, and hence we denote it by $h_{*} \mathscr{P}$. Then $h^{*}\left(S_{K}\right)$ 
forms a tame link in $h^{*}\left(O_{K}\right)$ with $\operatorname{im}\left(h^{*}\left(S_{K}\right)\right)=h^{-1}\left(\operatorname{im}\left(S_{K}\right)\right)$ by the construction of $h^{*}\left(O_{K}\right)$. We denote by $h^{*} K$ the arithmetic manifold $\left(h^{*}\left(O_{K}\right), h^{*}\left(S_{K}\right)\right)$.

A covering of $K$ is an arithmetic manifold $L=\left(O_{L}, S_{L}\right)$ equipped with an

$$
\iota_{L / K} \in \operatorname{Hom}_{\mathscr{C}_{0}}\left(O_{K}, O_{L}\right)
$$

such that $\left(\mathscr{F}_{0}\left(O_{L}\right), \mathscr{F}_{0}\left(\iota_{L / K}\right)\right)$ is a topological covering of $K$ with $L=\mathscr{F}_{0}\left(\iota_{L / K}\right)^{*} K$. In particular, $K$ itself is a covering of $K$ with respect to $\iota_{K / K}:=\mathrm{id}_{O_{K}}$. The notion of a covering of $K$ is partially analogous to a finite extension of a number field $k$.

Let $L$ be a covering of $K$. We put $h_{L / K}:=\mathscr{F}_{0}\left(\iota_{L / K}\right) \in \mathrm{C}\left(\mathscr{F}_{0}\left(O_{L}\right), M\right)$. For a $\mathscr{P} \in S_{L}$, we put $\iota_{L / K}^{*} \mathscr{P}:=\left(h_{L / K}\right)_{*} \mathscr{P}$. The compatible system of restrictions of $\iota_{L / K}$ gives a morphism $S_{K}^{-1} O_{K} \rightarrow S_{L}^{-1} O_{L}$ in $\widehat{\mathscr{C}}_{0}$. A morphism $\varphi$ in $\mathscr{C}_{0}$ is said to be unbranched if $\mathscr{F}_{0}(\varphi)$ is an unbranched covering of connected locally compact topological spaces of finite degree. We say that $L / K$ is unbranched if $\iota_{L / K}$ is unbranched. A covering $L^{\prime}$ of $K$ is said to be unbranched if $L^{\prime} / K$ is unbranched. The notion of an unbranched covering of $K$ is partially analogous to an unramified finite extension of $k$.

For coverings $L_{0}$ and $L_{1}$ of $K$, a covering morphism $L_{0} \rightarrow L_{1}$ over $K$ is a

$$
\sigma \in \operatorname{Hom}_{\mathscr{C}_{0}}\left(O_{L_{1}}, O_{L_{0}}\right)
$$

with $\iota_{L_{0} / K} \circ \sigma=\iota_{L_{1} / K}$ for which $L_{1}$ is a covering of $L_{0}$. In particular, for any covering $L$ of $K, \iota_{L / K}$ forms a covering morphism over $K$.

The set-theoretic composite of covering morphisms between coverings of $K$ again forms a covering morphism between coverings of $K$ in a contravariant way by the construction of the pullback. We denote by $\mathscr{C}_{0} / K$ the category of coverings of $K$ and covering morphisms over $K$. The correspondence $(N, \varphi) \leadsto \varphi^{*} K$ gives a contravariant functor Top $/ K \rightarrow \mathscr{C}_{0} / K$, which is fully faithful and essentially surjective by definition. The correspondence $L \leadsto S_{L}^{-1} O_{L}$ gives a functor $\mathscr{C}_{0} / K \rightarrow \widehat{\mathscr{C}}_{0}$ that is faithful because the image of a finite link is nowhere dense.

For a continuous map $h: X \rightarrow Y$ between topological spaces, we put $\operatorname{Aut}(h):=$ $\left\{g \in \operatorname{Aut}_{\text {Top }}(X) \mid h \circ g=h\right\}$. For any unbranched morphism $\varphi: O_{0} \rightarrow O_{1}$ in $\mathscr{C}_{0}$, every element of $\operatorname{Aut}\left(\mathscr{F}_{0}(\varphi)\right) \subset \operatorname{Aut}_{\text {Top }}\left(\mathscr{F}_{0}\left(O_{1}\right)\right)$ gives an automorphism of $O_{1}$ in $\mathscr{C}_{0}$ by the definition of the morphism class of $\mathscr{C}_{0}$. We extend this fact to a branched covering of arithmetic manifolds. For a group $G$, we denote by $G^{\text {op }}$ the opposite group of $G$. We put $\operatorname{Aut}(L / K):=\operatorname{Aut}\left(h_{L / K}\right)^{\text {op }}$. Every element of $\operatorname{Aut}(L / K)$ gives an automorphism $L \rightarrow L$ in $\mathscr{C}_{0} / K$ by the explicit presentation of $L=h_{L / K}^{*} K$ as the quotient of the disjoint union of an unbranched covering and finitely many standard branched coverings. Therefore $\operatorname{Aut}(L / K)$ acts on $L$ in $\mathscr{C}_{0} / K$. Since $\mathscr{F}_{0}$ is faithful, the map Aut $\mathscr{C}_{0} / K(L) \rightarrow$ $\operatorname{Aut}(L / K), g \mapsto \mathscr{F}_{0}(g)$ is a group isomorphism. In particular, $\operatorname{Aut}(L / K)$ admits a natural action on $S_{L}$.

For a branched covering $h$ of connected locally compact topological spaces, we say that $h$ is Galois if $\operatorname{Aut}(h)$ acts transitively on the fibre of every point of the unbranched covering associated with $h$, and is Abelian if $h$ is Galois and $\operatorname{Aut}(h)$ is Abelian. A morphism $\varphi$ in $\mathscr{C}_{0}$ is said to be Galois (resp. Abelian) if $\mathscr{F}_{0}(\varphi)$ is a Galois (resp. an Abelian) branched covering of connected locally compact topological spaces. We say that $L / K$ is Galois (resp. Abelian) or $L$ is Galois (resp. Abelian) as a covering of $K$ if $\iota_{L / K}$ is Galois (resp. Abelian). When $L / K$ is Galois, then we put $\operatorname{Gal}(L / K):=\operatorname{Aut}(L / K)$, 
and call it the Galois group of $L / K$. The notion of a Galois (resp. an Abelian) covering of $K$ is partially analogous to a Galois (resp. an Abelian) extension of $k$. Combining the facts that we will show in Proposition 3.2 (iii) and Theorem 3.4, it is easy to see that the profinite completion of $\mathrm{H}_{1}(M)$ controls unbranched Abelian coverings of $K$, and hence is partially analogous to the ideal class group $\mathscr{C} \ell_{k}$ of $k$.

\subsection{Analogue of a Number Field}

We say that $S_{K}$ is stably admissible (resp. stably generic) if $h^{*} S_{K}$ is admissible (resp. generic) for any topological covering $(N, h)$ of $K$, and that $K$ is stably admissible (resp. stably generic) if $S_{K}$ is stably admissible (resp. stably generic). If $K$ is stably admissible (resp. stably generic), then every covering of $K$ is stably admissible (resp. stably generic) by definition. The notion of an orientable closed stably generic arithmetic manifold is completely analogous to a number field.

Remark 2.2 We will verify the existence of a stably generic link in Theorem A.1. We note that the definition and the terminology of a stably admissible link are imitations of the notion of a very admissibility link, which was introduced and whose existence was verified by Niibo and Ueki in [NU]. The notion of a very admissible link in [NU] is equivalent to that of a countable stably admissible link for the case $\mathscr{C}=$ Top, therefore Theorem A.1 for the case $\mathscr{C}=$ Top follows from the corresponding result in [NU]. However, the notion of a very admissible link in the first version (arxiv:1501.03890v1 ) of [NU] was not formulated with the assumption of the tameness. The existence of a very admissible link without the tameness was verified in the first version of [NU] in January 2015, the existence of a countable stably admissible link was verified in our unpublished work in February 2016, and the existence of a very admissible link with the tameness was verified in their unpublished work in March 2016. Therefore our result is independent of their result. Furthermore, the existence of a stably generic link is much stronger than the existence of a stably admissible link, and hence than the existence of a very admissible link with the tameness.

\subsection{Analogue of an Algebraic Closure}

We fix an $\iota_{\infty} \in M \backslash \operatorname{im}\left(S_{K}\right)$. Let $Y$ be an element of $\operatorname{Cov}\left(M \backslash \operatorname{im}(s), \iota_{\infty}\right)$ (Example 1.5) for some $s \in \operatorname{Fin}\left(S_{K}\right)$. We denote by $\varphi_{Y}$ the canonical projection $Y \rightarrow M \backslash \operatorname{im}(s)$. Since $Y$ is a quotient of $\left(M \backslash \operatorname{im}(s), \iota_{\infty}\right)^{\sim}$, it is naturally equipped with a base point $*_{Y}$ with $\varphi_{Y}\left(*_{Y}\right)=\iota_{\infty}$.

We denote by $N_{Y}$ the Fox completion of $Y$ with respect to the composite of $\varphi_{Y}$ and the inclusion $M \backslash \operatorname{im}(s) \rightarrow M$ [Fox57, p. 245] and by the canonical projection $\bar{\varphi}_{Y}: N_{Y} \rightarrow M$. Then $\left(N_{Y}, \bar{\varphi}_{Y}\right)$ forms a topological covering of $K$. We obtain a covering $\bar{\varphi}_{Y}^{*} K$ of $K$.

We denote by $\operatorname{Cov}(K) \subset \bigsqcup_{s \in \operatorname{Fin}\left(S_{K}\right)} \operatorname{Cov}\left(M \backslash \operatorname{im}(s), \iota_{\infty}\right)$ the subset of $Y$ 's such that $\bar{\varphi}_{Y}$ is not unbranched at any point of $\operatorname{im}(s)$. Then $\operatorname{Cov}(K)$ forms a directed set with respect to the partial order $\leq$ given by setting $Y_{0} \leq Y_{1}$ if and only if $\varphi_{Y_{1}}$ factors through $\varphi_{Y_{0}}$. 
Let $\left(Y_{0}, Y_{1}\right) \in \operatorname{Cov}(K)^{2}$ with $Y_{0} \leq Y_{1}$. We denote by $\varphi_{Y_{1}, Y_{0}}: Y_{1} \rightarrow Y_{0}$ a unique local homeomorphism with $\varphi_{Y_{1}, Y_{0}}\left(*_{Y_{1}}\right)=*_{Y_{0}}$ and $\varphi_{Y_{0}} \circ \varphi_{Y_{1}, Y_{0}}=\varphi_{Y_{1}}$, and by $\bar{\varphi}_{Y_{1}, Y_{0}}: N_{Y_{1}} \rightarrow$ $N_{Y_{0}}$ a unique continuous extension of $\varphi_{Y_{1}, Y_{0}}$. We have

$$
\bar{\varphi}_{Y_{1}, Y_{0}} \in \operatorname{Hom}_{\text {Top } / K}\left(\left(N_{Y_{1}}, \bar{\varphi}_{Y_{1}}\right),\left(N_{Y_{0}}, \bar{\varphi}_{Y_{0}}\right)\right) \text {. }
$$

So $\left(\bar{\varphi}_{Y}^{*} K\right)_{Y \in \operatorname{Cov}(K)}$ forms an object of $\operatorname{Ind}\left(\mathscr{C}_{0} / K\right)$ with respect to $\left(\bar{\varphi}_{Y_{0}, Y_{1}}\right)_{Y_{0} \leq Y_{1}}$.

We denote by $M(K) \subset \operatorname{ob}\left(\mathscr{C}_{0} / K\right)$ the subset $\left\{\bar{\varphi}_{Y}^{*} K \mid Y \in \operatorname{Cov}(K)\right\}$. The map $\operatorname{Cov}(K) \rightarrow M(K), Y \mapsto \bar{\varphi}_{Y}^{*}$ is bijective by definition. Therefore, $(L)_{L} \in M(K)$ forms an object $\bar{K}$ of $\operatorname{Ind}\left(\mathscr{C}_{0} / K\right)$ naturally isomorphic to $\left(\bar{\varphi}_{Y}^{*} K\right)_{Y \in \operatorname{Cov}(K)}$ in $\operatorname{Ind}\left(\mathscr{C}_{0} / K\right)$, which is analogous to the algebraic closure $\bar{k}$ in $\mathbb{C}$ of a number field $k$ equipped with an embedding $i_{\infty}: k \hookrightarrow \mathbb{C}$, and call it the algebraic closure of $K$ with respect to $\iota_{\infty}$.

We denote by $M^{\mathrm{Gal}}(K) \subset M(K)$ the subset of Galois coverings of $K$, and by Grp the category of groups and group homomorphisms. We will prove in Corollary 3.3 that $M^{\mathrm{Gal}}(K)$ is cofinal in $M(K)$, and the system $(\mathrm{Gal}(L / K))_{L \in M^{\mathrm{Gal}}(K)}$ naturally forms an object of Pro(Grp) with surjective transition maps. We denote by $G_{K}$ the profinite group given as the projective limit of $(\operatorname{Gal}(L / K))_{L \in M^{\mathrm{Gal}}(K)}$, where $\operatorname{Gal}(L / K)$ is equipped with the discrete topology for each $L \in M^{\mathrm{Gal}}(K)$. Then the underlying group of $G_{K}$ naturally acts on $\bar{K}$ in $\operatorname{Ind}\left(\mathscr{C}_{0} / K\right)$.

Let $\wp \in S_{K}$. A place of $\bar{K}$ over $\wp$ is a system $\left(\wp_{L}\right)_{L \in M(K)} \in \prod_{L \in M(K)} S_{L}$ with $\iota_{L_{1} / L_{0}}^{*} \wp_{L_{1}}=\wp_{L_{0}}$ for any $\left(L_{0}, L_{1}\right) \in M(K)$ with $L_{0} \leq L_{1}$. The notion of a place of $\bar{K}$ over $\wp$ is analogous to a place $\widehat{p}$ of $\bar{k}$ over a maximal ideal of the ring of algebraic integers in $k$.

Let $\widehat{\wp}=\left(\wp_{L}\right)_{L \in M(K)}$ be a place of $\bar{K}$ over $\wp$. Then the system $\left(L_{\wp_{L}}\right)_{L \in M(K)}$ forms an object of $\operatorname{Ind}\left(\widehat{\mathscr{C}}_{0}\right)=\operatorname{Ind}\left(\operatorname{Ind}\left(\mathscr{C}_{0}\right)\right)$ with respect to $\left(\iota_{\wp_{L_{1}} / \wp_{L_{0}}}\right)_{L_{0} \leq L_{1}}$, and naturally gives an object $\bar{K}_{\widehat{\wp}}$ of $\operatorname{Ind}\left(\mathscr{C}_{0}\right)=\widehat{\mathscr{C}}_{0}$ that is analogous to the algebraic closure of $k_{p}$ given as the completion $\bar{k}_{P}$ of $\bar{k}$ at $P$.

\subsection{Analogues of the Multiplicative Groups}

We introduce two analogues of the multiplicative groups of number fields. We denote by $\Delta$ the simplex category, and by $\mathrm{Ab} \subset$ Grp the full subcategory of Abelian groups. The correspondence $[n] \leadsto \mathscr{A}_{\Delta^{n}}$ gives a contravariant functor $\mathscr{A}_{\Delta} \bullet: \Delta \rightarrow \mathscr{C}_{0}$, because coface maps and codegeneracy maps are $\mathscr{C}$-morphisms.

For an $X \in \mathrm{ob}\left(\mathscr{C}_{0}\right)$, we denote by $\mathrm{C}_{\mathrm{m}}^{*}(X)=\left(\mathrm{C}_{\mathrm{m}}^{n}(X), d^{n}\right)_{n \in \mathbb{N}}$ the cochain complex $\left(\mathbb{Z}^{\operatorname{Hom}_{\mathscr{C}}\left(X, \mathscr{A}_{\Delta^{n}}\right)}\right)_{n \in \mathbb{N}}$ that is naturally identified with

$$
\left(\operatorname{Hom}_{\mathrm{Ab}}\left(\mathbb{Z}^{\oplus \operatorname{Hom}_{\mathscr{C}}\left(X, \mathscr{A}_{\Delta^{n}}\right)}, \mathbb{Z}\right)\right)_{n \in \mathbb{N}},
$$

associated with the composite of $\mathscr{A}_{\Delta}$ and the inclusion $\mathscr{C}_{0} \hookrightarrow \mathscr{C}$, by $\left(\mathrm{Z}_{\mathrm{m}}^{n}(X)\right)_{n \in \mathbb{N}}$ the system $\left(\operatorname{ker}\left(d^{n}\right)\right)_{n \in \mathbb{N}}$ of cocycles of $\mathrm{C}_{\mathrm{m}}^{*}(X)$, by $\left(\mathrm{B}_{\mathrm{m}}^{n}(X)\right)_{n \in \mathbb{N}}$ the system $\left(\operatorname{im}\left(d^{n}\right)\right)_{n \in \mathbb{N}}$ of coboundaries of $\mathrm{C}_{\mathrm{m}}^{*}(X)$, and by $\left(\mathrm{H}_{\mathrm{m}}^{n}(X)\right)_{n \in \mathbb{N}}$ the cohomology of $\mathrm{C}_{\mathrm{m}}^{*}(X)$. For any $n \in \mathbb{N}$, the correspondences $X \leadsto \mathrm{C}_{\mathrm{m}}^{n}(X), \mathrm{Z}_{\mathrm{m}}^{n}(X), \mathrm{B}_{\mathrm{m}}^{n}(X), \mathrm{H}_{\mathrm{m}}^{n}(X)$ give functors $\mathrm{C}_{\mathrm{m}}^{n}, \mathrm{Z}_{\mathrm{m}}^{n}, \mathrm{~B}_{\mathrm{m}}^{n}, \mathrm{H}_{\mathrm{m}}^{n}: \mathscr{C} \rightarrow \mathrm{Ab}$. We note that they are covariant because the source and the target of a morphism in $\mathscr{C}$ are defined in a contravariant way relative to Top.

For a covariant functor $F: \mathscr{C} \rightarrow \mathrm{Ab}$, we denote by $\widehat{F}$ the Kan extension of $F$ along the embedding $\mathscr{C} \hookrightarrow \widehat{\mathscr{C}}$, i.e., the natural extension $\widehat{\mathscr{C}} \rightarrow \operatorname{Ab}$ of $F$ sending $\left(X_{j}\right)_{j \in J} \in \operatorname{ob}(\widehat{\mathscr{C}})$ 
to $\lim _{\longrightarrow \in J} F\left(X_{j}\right)$. The functors $\widehat{Z}_{\mathrm{m}}^{1}$ and $\mathrm{C}_{\mathrm{m}}^{1}$ are analogous to the multiplicative group functors $\mathscr{O}^{\times}$and $\mathscr{M}^{\times}$of regular sections and meromorphic sections on algebraic varieties, respectively. Indeed, we will verify local class field theory for $\widehat{Z}_{m}^{1}$ in Theorem 3.15 and Hilbert's Theorem 90 for $\mathrm{C}_{\mathrm{m}}^{1}$ in Theorem 3.7. We note that we have a natural equivalence $\widehat{\mathrm{Z}}_{\mathrm{m}}^{n} / \widehat{\mathrm{B}}_{\mathrm{m}}^{n} \rightarrow \widehat{\mathrm{H}}_{\mathrm{m}}^{n}$ for each $n \in \mathbb{N}$ by the exactness of the inductive limit.

We put $K^{\times}:=\widehat{\mathrm{Z}}_{\mathrm{m}}^{1}\left(S_{K}^{-1} O_{K}\right)\left(\right.$ Section 2.2) and $\bar{K}^{\times}:=\widehat{\mathrm{Z}}_{\mathrm{m}}(\bar{K})$ (Section 2.5), and call them the multiplicative groups of $K$ and $\bar{K}$ respectively. We denote by $O_{K}^{\times} \subset K^{\times}$the image of the canonical homomorphism $\mathrm{Z}_{\mathrm{m}}^{1}\left(O_{K}\right) \rightarrow K^{\times}$, which, unfortunately, is not injective unless $S_{K}=\varnothing$, and call it the unit group of $K$. Then $K^{\times}$(resp. $O_{K}^{\times}$) is analogous to the multiplicative group $k^{\times}$(resp. the unit group $\mathscr{O}_{k}^{\times}$) of a number field $k$. The correspondence $L \leadsto L^{\times}\left(\right.$resp. $\left.L \leadsto O_{L}^{\times}\right)$gives a functor $\mathscr{C}_{0} / K \rightarrow \mathrm{Ab}$.

Remark 2.3 In the preceding formulation in [NU], a counterpart of the multiplicative group is given as the inductive limit of the second singular homology groups $\mathrm{H}_{2}$ with respect to a system of group homomorphisms defined in a geometric way. Through the Poincaré duality, it can be interpreted as $\widehat{\mathrm{H}}_{\mathrm{m}}^{1}$, which is defined in an algebraic way from $\mathrm{H}_{\mathrm{m}}^{1}$. Therefore our formulation of $K^{\times}$is a refinement of the algebraic interpretation $\widehat{\mathrm{H}}_{\mathrm{m}}^{1}$ of the counterpart of the multiplicative group in [NU]. We will show in Theorem 3.8 that $K^{\times}$satisfies the descent property, which justifies our formulation compared to the original one in $[\mathrm{NU}]$.

Let $\wp \in S_{K}$. We put $K_{\wp}^{\times}:=\widehat{Z}_{\mathrm{m}}^{1}\left(K_{\wp}\right)$ (Example 2.2). We denote by $\iota_{\wp}^{\times}: K^{\times} \rightarrow K_{\wp}^{\times}$the group homomorphism associated with the morphism $S_{K}^{-1} O_{K} \rightarrow K_{\wp}$ in $\widehat{\mathscr{C}}_{0}$ given by the compatible system of restrictions of $\operatorname{id}_{M}$.

For a covering $L$ of $K$ and a $\mathscr{P} \in S_{L}$ with $\iota_{L / K}^{*} \mathscr{P}=\wp$, the compatible system of restrictions of $h_{L / K}$ gives morphisms $\left(O_{K}\right)_{\wp} \rightarrow\left(O_{L}\right)_{\mathscr{P}}$ and $\iota_{\mathscr{P} / \wp}: K_{\wp} \rightarrow L_{\mathscr{P}}$ in $\widehat{\mathscr{C}}_{0}$.

Let $\widehat{\wp}=\left(\wp_{L}\right)_{L \in M(K)}$ be a place of $\bar{K}$ over $\wp$. Since $\left(L_{\wp_{L}}\right)_{L \in M(K)}$ forms an inductive system in $\widehat{\mathscr{C}}_{0},\left(L_{\wp_{L}}^{\times}\right)_{L \in M(K)}$ forms an inductive system in Grp, by the functoriality of $\widehat{Z}_{\mathrm{m}}^{1}$. We put $\bar{K}_{\widehat{\wp}}^{\times}:=\widehat{Z}_{\mathrm{m}}^{1}\left(\bar{K}_{\widehat{\wp}}\right)$. By the definition of $\bar{K}_{\widehat{\wp}}, \bar{K}_{\widehat{\wp}}^{\times}$is naturally isomorphic to the inductive limit of $\left(L_{\wp_{L}}^{\times}\right)_{L \in M(K)}$ in Ab.

\subsection{Analogue of the $p$-adic Valuation}

In order to introduce several homomorphisms, we compare $\mathrm{H}_{\mathrm{m}}^{1}$ and $\mathrm{H}^{1}$. We denote by $\Theta_{O}$ the group homomorphism $\mathrm{H}^{1}\left(\mathscr{F}_{0}(O)\right) \rightarrow \mathrm{H}_{\mathrm{m}}^{1}(O)$ induced by the restriction $\operatorname{map} \mathbb{Z}^{\mathrm{C}\left(\Delta^{1}, \mathscr{F}_{0}(O)\right)} \rightarrow \mathrm{C}_{\mathrm{m}}^{1}(O),\left(c_{\gamma}\right)_{\gamma \in \mathrm{C}\left(\Delta^{1}, \mathscr{F}_{0}(O)\right)} \mapsto\left(c_{\mathscr{F}}(\chi)\right)_{\chi \in \operatorname{Hom}_{\mathscr{C}}\left(O, \mathscr{A}_{\Delta^{1}}\right)}$.

Proposition 2.4 For any strict $\mathscr{C}$-manifold $X$, the homomorphism $\Theta_{\mathscr{A}_{X}}: \mathrm{H}^{1}(X) \longrightarrow$ $\mathrm{H}_{\mathrm{m}}^{1}\left(\mathscr{A}_{\mathrm{X}}\right)$ is a group isomorphism.

Proof We denote by $C^{*}=\left(C^{n}, \delta^{n}\right)_{n \in \mathbb{N}}$ the singular cochain complex of $X$, and by $\rho=\left(\rho_{n}\right)_{n \in \mathbb{N}}$ the cochain map $C^{*} \rightarrow \mathrm{C}_{\mathrm{m}}^{*}\left(\mathscr{A}_{X}\right)$ given by restriction maps. Then $\Theta_{\mathscr{A}_{X}}$ in given as $\mathrm{H}^{1}(\rho)$. By definition, $\rho_{0}$ is a group isomorphism. 
We show the injectivity of $\mathrm{H}^{1}(\rho)$. For any $\gamma \in \mathrm{C}\left(\Delta^{1}, X\right)$, there is a homotopy $\Delta^{1} \times[0,1] \rightarrow X$ relative to $\partial \Delta^{1}$ connecting $\gamma$ and a map $\Delta^{1} \rightarrow X$ of class $C^{\infty}$ by Whitney's approximation

theorem [Lee03, Theorem 10.21] applied to the continuous extension $\widetilde{\gamma}:\left\{\left(t_{0}, t_{1}\right) \epsilon\right.$ $\left.\mathbb{R}^{2} \mid t_{0}+t_{1}=1\right\} \rightarrow X$ of $\gamma$ given by setting $\widetilde{\gamma}\left(t_{0}, t_{1}\right)=\widetilde{\gamma}(1,0)$ for any $\left(t_{0}, t_{1}\right) \in \mathbb{R}^{2}$ with $t_{0}+t_{1}=1 \leq t_{0}$ and $\widetilde{\gamma}\left(t_{0}, t_{1}\right)=\widetilde{\gamma}(0,1)$ for any $\left(t_{0}, t_{1}\right) \in \mathbb{R}^{2}$ with $t_{0}+t_{1}=1 \leq t_{1}$. It implies that $\mathrm{H}^{1}(\rho)$ is injective because $\rho_{0}$ is surjective.

We show the surjectivity of $\mathrm{H}^{1}(\rho)$. Let $\widetilde{c} \in \mathrm{H}_{\mathrm{m}}^{1}\left(\mathscr{A}_{X}\right)$. We take a representative $c \in \mathrm{Z}_{\mathrm{m}}^{1}\left(\mathscr{A}_{X}\right)$ of $\bar{c}$. We construct a $c^{\prime} \in \operatorname{ker}\left(\delta^{1}\right)$ with $\rho_{1}\left(c^{\prime}\right)=c$. Let $\gamma \in \mathrm{C}\left(\Delta^{1}, X\right)$. By Whitney's approximation theorem again, there is a homotopy map $\Phi: \Delta^{1} \times[0,1] \rightarrow X$ relative to $\partial \Delta^{1}$ connecting $\gamma$ and a $C^{\infty}$-map $\widetilde{\gamma}: \Delta^{1} \rightarrow X$. By Whitney's approximation theorem for smooth homotopy [Lee03, Proposition 10.22], two smooth paths in $X$ sharing endpoints and admitting a homotopy map relative to $\partial \Delta^{1}$ connecting them, admit a smooth homotopy map relative to $\partial \Delta^{1}$ connecting them, and hence $c_{\gamma}:=$ $c(\widetilde{\gamma}) \in \mathbb{Z}$ depends only on $\gamma$. We put $c^{\prime}:=\left(c_{\gamma}\right)_{\gamma \in \mathrm{C}\left(\Delta^{1}, X\right)} \in C^{1}$. Then we have $\rho_{1}\left(c^{\prime}\right)=c$ by the construction.

Let $\sigma \in \mathrm{C}\left(\Delta^{2}, X\right)$. We put $\partial[\sigma]=\sum_{i=0}^{2}(-1)^{i}\left[\partial_{i} \sigma\right] \in \mathbb{Z}^{\oplus \mathrm{C}\left(\Delta^{1}, X\right)}$ with

$$
\left(\partial_{i} \sigma\right)_{i=0}^{2} \in \mathrm{C}\left(\Delta^{1}, X\right)^{3} \text {. }
$$

For each $i \in\{0,1,2\}$, there is a homotopy $\Delta^{1} \times[0,1] \rightarrow X$ relative to $\partial \Delta^{1}$ connecting $\partial_{i} \gamma$ and a $\gamma_{i} \in \operatorname{Hom}_{\mathrm{C}^{\infty}}\left(\Delta^{1}, X\right) \subset \mathrm{C}\left(\Delta^{1}, X\right)$ by Whitney's approximation theorem applied to a continuous extension of $\partial_{i} \sigma$. Then $\sum_{i=0}^{2}(-1)^{i}\left[\gamma_{i}\right] \in \mathbb{Z}^{\oplus \mathrm{C}\left(\Delta^{1}, X\right)}$ lies in $\sum_{i=0}^{2}\left((-1)^{i}\left[\partial_{i} \sigma\right]+\partial \mathbb{Z}^{\oplus \mathrm{C}\left(\Delta^{2}, X\right)}\right)=\partial[\sigma]+\partial \mathbb{Z}^{\oplus \mathrm{C}\left(\Delta^{2}, X\right)}=\partial \mathbb{Z}^{\oplus \mathrm{C}\left(\Delta^{2}, X\right)}$, and hence in $\partial \mathbb{Z}^{\oplus \operatorname{Hom}_{\mathrm{C}}\left(\Delta^{2}, X\right)}$ by the comparison theorem between the smooth singular homology and the singular homology [Lee03, Theorem 16.6]. Therefore, we obtain $\delta^{1}\left(c^{\prime}\right)(\sigma)=$ $\sum_{i=0}^{2}(-1)^{i} c^{\prime}\left(\partial_{i} \sigma\right)=\sum_{i=0}^{2}(-1)^{i} c\left(\gamma_{i}\right)=0$. It implies $\delta^{1}\left(c^{\prime}\right)=0$. We conclude that $\mathrm{H}^{1}(\rho)$ is bijective.

For an $\left(r, r^{\prime}\right) \in(0,1]^{2}$ with $r^{\prime}<r$, we denote by $\mu_{r, r^{\prime}}$ (resp. $\left.\lambda_{r, r^{\prime}}\right)$ the morphism $\mathscr{A}_{V(r)} \rightarrow \mathscr{A}_{\Delta^{1}}$ in $\mathscr{C}$ given by the $\mathscr{C}$-morphism $\Delta^{1} \rightarrow V(r),\left(t_{0}, t_{1}\right) \mapsto\left(1, r^{\prime} \theta\left(t_{1}\right)\right)$ (resp. $\left.\left(t_{0}, t_{1}\right) \mapsto\left(\theta\left(t_{1}\right), r^{\prime}\right)\right)$, and by $\mu_{r, r^{\prime}}^{\vee}\left(\right.$ resp. $\left.\lambda_{r, r^{\prime}}^{\vee}\right)$, the group homomorphism $\mathrm{Z}_{\mathrm{m}}^{1}\left(\mathscr{A}_{V(r)}\right) \rightarrow \mathbb{Z}$ given by the evaluation at $\mu_{r, r^{\prime}}\left(\right.$ resp. $\left.\lambda_{r, r^{\prime}}\right)$. We verify that the system $\left(\mu_{r, 2^{-1} r}^{\vee}\right)_{r \in(0,1]}\left(\right.$ resp. $\left.\left(\lambda_{r, 2^{-1} r}^{\vee}\right)_{r \in(0,1]}\right)$ yields a group homomorphism $\widehat{Z}_{\mathrm{m}}^{1}\left(\mathbb{T}^{2}\right) \rightarrow \mathbb{Z}$.

Proposition 2.5 Let $\left(r, r^{\prime}, r^{\prime \prime}\right) \in(0,1]^{3}$ with $r^{\prime \prime}<r^{\prime}<r$. For any $c \in \mathrm{Z}_{\mathrm{m}}^{1}\left(\mathscr{A}_{V(r)}\right)$, the equality $c\left(\mu_{r, r^{\prime}}\right)=c\left(\mu_{r, r^{\prime \prime}}\right)=\left(\left.c\right|_{V\left(r^{\prime}\right)}\right)\left(\mu_{r^{\prime}, r^{\prime \prime}}\right)$, respectively, $c\left(\lambda_{r, r^{\prime}}\right)=c\left(\lambda_{r, r^{\prime \prime}}\right)=$ $\left(\left.c\right|_{V\left(r^{\prime}\right)}\right)\left(\lambda_{r^{\prime}, r^{\prime \prime}}\right)$, holds, where $\left.c\right|_{V\left(r^{\prime}\right)}$ denotes the image of $c$ in $\mathrm{Z}_{\mathrm{m}}^{1}\left(V\left(r^{\prime}\right)\right)$.

Proof The assertion follows from Proposition 2.4 applied to $V(r)$ and $V\left(r^{\prime}\right)$, because $\left[\mathscr{F}\left(\mu_{r, r^{\prime}}\right)\right]-\left[\mathscr{F}\left(\mu_{r, r^{\prime \prime}}\right)\right] \in \mathrm{Z}^{\oplus \mathrm{C}\left(\Delta^{1}, V(r)\right)}\left(\operatorname{resp} .\left[\mathscr{F}\left(\lambda_{r, r^{\prime}}\right)\right]-\left[\mathscr{F}\left(\lambda_{r, r^{\prime \prime}}\right)\right] \epsilon\right.$ $\left.\mathrm{Z}^{\oplus \mathrm{C}\left(\Delta^{1}, V(r)\right)}\right)$ is a singular 1-boundary.

By Proposition 2.5, the system $\left(\mu_{r, 2^{-1} r}^{\vee}\right)_{r \in(0,1]}\left(\right.$ resp. $\left.\left(\lambda_{r, 2^{-1} r}^{\vee}\right)_{r \in(0,1]}\right)$ yields a group homomorphism $\widehat{Z}_{\mathrm{m}}^{1}\left(\mathbb{T}^{2}\right) \rightarrow \mathbb{Z}$ that we denote by $\mu^{\vee}$ (resp. $\lambda^{\vee}$ ) and that is obviously 
surjective by an argument similar to the singular cohomology using the perfect pairing with the singular homology. We show that $\mu^{\vee}$ (resp. $\lambda^{\vee}$ ) is stable under the action of the group of automorphisms of $\mathbb{S}^{1}$ over $\mathscr{A}_{\mathrm{S}^{1}}$.

Proposition 2.6 Let $\left(r, r^{\prime}\right) \in(0,1]^{2}$. For any local isomorphism $\sigma: \mathscr{A}_{V\left[r^{\prime}\right)} \rightarrow \mathscr{A}_{V[r)}$ in $\mathscr{C}$ with $\mathscr{F}(\sigma)(z, 0)=(z, 0)$ for any $z \in S^{1}$, we have $\mu^{\vee} \circ \widehat{Z}_{\mathrm{m}}\left(\sigma^{\circ}\right)=\mu^{\vee}$, (resp., $\left.\lambda^{\vee} \circ \widehat{Z}_{\mathrm{m}}\left(\sigma^{\circ}\right)=\lambda^{\vee}\right)$, where $\sigma^{\circ}$ is the automorphism of $\mathbb{T}^{2}$ in $\widehat{\mathscr{C}}$ given by the compatible system of restrictions of $\mathscr{F}(\sigma)$.

Proof By Proposition 2.5, we may replace $r$ by a sufficiently smaller one, and assume that $\mathscr{F}(\sigma)$ is injective. Then $\sigma$ induces a local isomorphism $\left.\sigma\right|_{V\left(r^{\prime}\right)}: \mathscr{A}_{V\left(r^{\prime}\right)} \rightarrow \mathscr{A}_{V(r)}$ in $\mathscr{C}$. The assertion follows from Proposition 2.4 applied to $V(r)$ and $V\left(r^{\prime}\right)$, because $\left[\mathscr{F}\left(\mu_{r^{\prime}, 2^{-1} r^{\prime}}\right)\right]-\left[\mathscr{F}\left(\left.\mu_{r, 2^{-1} r} \circ \sigma\right|_{V\left(r^{\prime}\right)}\right)\right] \in \mathrm{Z}^{\oplus \mathrm{C}\left(\Delta^{1}, V\left(r^{\prime}\right)\right)}\left(\operatorname{resp} .\left[\mathscr{F}\left(\lambda_{r^{\prime}, 2^{-1} r^{\prime}}\right)\right]-\right.$ $\left.\left[\mathscr{F}\left(\left.\lambda_{r, 2^{-1} r} \circ \sigma\right|_{V\left(r^{\prime}\right)}\right)\right] \in \mathrm{Z}^{\oplus \mathrm{C}\left(\Delta^{1}, V\left(r^{\prime}\right)\right)}\right)$ is a singular 1-boundary.

Let $\wp \in S_{K}$. The tameness of $\wp$ ensures that $K_{\wp}^{\times}$admits a group isomorphism to $\widehat{Z}_{\mathrm{m}}^{1}\left(\mathbb{T}^{2}\right)$ depending on a choice of a tubular neighbourhood of $\wp$. By Proposition 2.6, the composite $v_{\wp}$ (resp. $v_{\wp}^{\log }$ ) of the isomorphism $K_{\wp}^{\times} \rightarrow \widehat{Z}_{\mathrm{m}}^{1}\left(\mathbb{T}^{2}\right)$ and $\mu^{\vee}$ (resp. $\lambda^{\vee}$ ) is independent of the choice of a tubular neighbourhood of $\wp$ and is analogous to the $p$-adic valuation (resp. the composition of the $p$-adic valuation and the Iwasawa logarithm $k_{p}^{\times} \rightarrow k_{p}$ ) of the completion $k_{p}$ of a number field $k$ at a maximal ideal $p$ of the ring of algebraic integers in $k$. We call $v_{\wp}$ the valuation of $K_{\wp}^{\times}$.

We put $\left(O_{K}\right)_{\wp}^{\times}:=\operatorname{ker}\left(v_{\wp}\right) \subset K_{\wp}^{\times}$. We note that $\mathscr{F}$ induces a group isomorphism $\mathbb{Z} \cong \mathrm{H}^{1}\left(\mathrm{~S}^{1}\right) \rightarrow \widehat{\mathrm{H}}_{\mathrm{m}}^{1}\left(\mathbb{S}^{1}\right) \cong \widehat{\mathrm{H}}_{\mathrm{m}}^{1}\left(\left(O_{K}\right)_{\wp}\right)$ by Proposition 2.4 applied to $V[1)$. Although we do not use this fact, it is easy to see, through the isomorphism, that the group homomorphism $\widehat{Z}_{\mathrm{m}}^{1}\left(\left(O_{K}\right)_{\wp}\right) \rightarrow \widehat{Z}_{\mathrm{m}}^{1}\left(K_{\wp}\right)=K_{\wp}^{\times}$associated with the canonical morphism $\mathbb{S}^{1} \rightarrow \mathbb{T}^{2}$ in $\widehat{\mathscr{C}}_{0}$ induces a surjective group homomorphism $\widehat{Z}_{\mathrm{m}}^{1}\left(\left(O_{K}\right)_{\wp}\right) \rightarrow\left(O_{K}\right)_{\wp}^{\times}$ that is, unfortunately, not injective.

\subsection{Analogue of the Reduction}

Let $L$ be a Galois covering of $K$. We fix a $\wp \in S_{K}$. For a $\mathscr{P} \in S_{L}$ with $\iota_{L / K}^{*} \mathscr{P}=\wp$, we denote by $\operatorname{Gal}(L / K) \mathscr{P} \subset \operatorname{Gal}(L / K)$ the stabiliser subgroup of $\mathscr{P}$ with respect to the action of $\mathrm{Gal}(L / K)$ on $S_{L}$, and call it the decomposition group of $L / K$ at $\mathscr{P}$. Since $\mathrm{Gal}(L / K)$ acts transitively on the fibre of each point of $\mathscr{F}_{0}\left(O_{L}\left[h_{L / K}^{*}(s)^{-1}\right]\right)$ for a sufficiently large $s \in \operatorname{Fin}\left(S_{K}\right), \operatorname{Gal}(L / K)$ acts transitively on $\iota_{L / K}^{-1}(\{\wp\}) \subset S_{L}$. Therefore, for any $\left(\mathscr{P}_{0}, \mathscr{P}_{1}\right) \in S_{L}^{2}$ with $\iota_{L / K}^{*} \mathscr{P}_{0}=\iota_{L / K}^{*} \mathscr{P}_{1}=\wp, \operatorname{Gal}(L / K)_{\mathscr{P}_{0}}$ and $\operatorname{Gal}(L / K) \mathscr{P}_{1}$ are conjugate to each other.

We fix a $\mathscr{P} \in S_{L}$ with $\iota_{L / K}^{*} \mathscr{P}=\wp$. By the definition of a covering, $h_{L / K}$ is locally presented as the standard branched covering $h_{d, e, f}[r)$ with $r \in(0,1]$ and

$$
(d, e, f) \in \mathbb{Z} \times \mathbb{N}_{>0} \times \mathbb{N}_{>0}
$$


around $\operatorname{im}(\mathscr{P})$, and the restriction $\operatorname{im}(\mathscr{P}) \rightarrow \operatorname{im}(\wp)$ of $h_{L / K}$ is an unbranched covering corresponding to the map $S^{1} \rightarrow S^{1}, z \mapsto z^{f}$ through the presentation. It implies that the automorphism group of $\operatorname{im}(\mathscr{P})$ over $\operatorname{im}(\wp)$ in Top is canonically isomorphic to $\mathbb{Z} / f \mathbb{Z}$ by the definition of a knot, using $\operatorname{Aut}_{\mathscr{C}}^{+}\left(\mathscr{A}_{\mathrm{S}^{1}}\right) \subset \operatorname{Aut}_{\mathscr{C}}\left(\mathscr{A}_{\mathrm{S}^{1}}\right)$. In particular, $f_{\mathscr{P} / \wp \circ}:=f$ depends only on $\iota_{L / K}$ and $\mathscr{P}$. We obtain a group homomor-

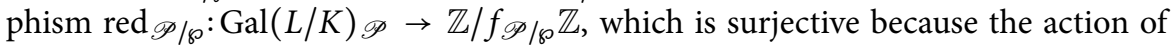
$\operatorname{Gal}(L / K)_{\mathscr{P}}$ on each fibre of the restriction of $h_{L / K}$ to sufficiently small neighbourhoods of $\operatorname{im}(\wp)$ and $\operatorname{im}(\mathscr{P})$ is transitive. Since $e f_{\mathscr{P} / \wp}$ coincides with the degree of the unbranched covering associated with the restriction of $h_{L / K}$ to neighbourhoods of $\mathscr{P}$ and $\wp, e_{\mathscr{P} / \wp}:=e$ also depends only on $\iota_{L / K}$ and $\mathscr{P}$. The indices $f_{\mathscr{P} / \wp}$ and $e_{\mathscr{P} / \wp}$ are analogous to the inertia degree and the ramification index of an extension of local fields.

Remark 2.7 If $\mathscr{C}=\mathrm{PLSp}^{\text {op }}$, then the equality $\# O_{L} / \mathscr{P}=\left(\# O_{K} / \wp\right)^{f_{\mathscr{P} / 8}}($ Section 2.1) holds.

We fix a place $\widehat{\wp}=\left(\wp_{L}\right)_{L \in M(K)}$ of $\bar{K}$ over $\wp$. Then the system $\left(\mathrm{Gal}(L / K)_{\wp_{L}}\right)_{L \in M^{\mathrm{Gal}}(K)}$ forms an object of Pro(Grp) with respect to the transition maps of

$$
(\operatorname{Gal}(L / K))_{L \in M^{\mathrm{Gal}}(K)} \text {. }
$$

We denote by $G_{K, \widehat{\wp}}$ the profinite group given as its projective limit of

$$
\left(\operatorname{Gal}(L / K)_{\wp_{L}}\right)_{L \in M^{\mathrm{Gal}}(K)},
$$

whose underlying group of $G_{K, \widehat{\wp}}$ naturally acts on $\bar{K}_{\widehat{\wp}}$ in $\widehat{\mathscr{C}}_{0}$ by definition. For any $\left(L, L^{\prime}\right) \in M^{\mathrm{Gal}}(K)^{2}$ with $L^{\prime} \leq L$, we have $f_{\wp_{L^{\prime}} / \wp_{\wp}} \mid f_{\wp_{L} / \wp_{\wp}}$, and the composite of the canonical projection $\operatorname{Gal}(L / K)_{\wp_{L}} \rightarrow \operatorname{Gal}\left(L^{\prime} / K\right)_{\wp_{L^{\prime}}}$ with $\operatorname{red}_{\wp_{L^{\prime}} / \wp}$ coincides with the composite of $\operatorname{red}_{\wp_{L} / \wp}$ with the canonical projection $\mathbb{Z} / f_{\wp_{L} / \wp^{\prime}} \mathbb{Z} \rightarrow \mathbb{Z} / f_{\wp_{L^{\prime}} / \wp} \mathbb{Z}$, by definition. Therefore, we obtain a continuous group homomorphism

$$
G_{K, \widehat{\wp}} \longrightarrow \lim _{L \in M^{\text {Gal }}(K)} \mathbb{Z} / f_{\wp_{L} / \wp} \mathbb{Z}
$$

which is surjective by the compactness of $G_{K, \widehat{\wp}}$.

In particular, we obtain a surjective continuous group homomorphism

$$
d_{\widehat{\wp}}: G_{K, \widehat{\wp}} \longrightarrow \widehat{\mathbb{Z}}
$$

under the assumption that for any $n \in \mathbb{N}_{>0}$, there is an $L \in M(K)$ with $n \mid f_{\wp_{L} / \wp}$. We note that this condition obviously holds when $K$ is presented as $\left(\mathscr{A}_{V[r}\right.$, $\left.\{\wp\}\right)$ for some $r \in(0,1]$ and $\wp$ is represented by the morphism $\mathscr{A}_{V[r)} \rightarrow \mathscr{A}_{\Delta^{1}}$ in $\mathscr{C}$ given by the $\mathscr{C}$-morphism $\mu_{r}: \Delta^{1} \rightarrow V[r),\left(t_{0}, t_{1}\right) \mapsto\left(\theta\left(t_{1}\right), 0\right)$, but does not necessarily hold in general. The homomorphism $d_{\widehat{\varsigma}}$ is analogous to the reduction map from the absolute Galois group of a $p$-adic field to the absolute Galois group of the residue field.

\subsection{Analogue of the Idèle Class Group}

We denote by $I_{K}$ the restricted product of $\left(K_{\wp}^{\times}\right)_{\wp \in S_{K}}$ with respect to $\left(\left(O_{K}\right)_{\wp}^{\times}\right)_{\wp \in S_{K}}$, i.e., the subgroup of $\prod_{\wp \in S_{K}} K_{\wp}^{\times}$consisting of $\left(c_{\wp}\right)_{\wp \in S_{K}}$ 's with $\#\left\{\wp \in S_{K} \mid c_{\wp} \notin\left(O_{K}\right)_{\wp}^{\times}\right\}<\infty$, and call it the idèle group of $K$. Then $I_{K}$ is analogous to the idèle group $\mathscr{I}_{k}$ of a number 
field $k$. Unlike $\mathscr{I}_{k}$, we use the additive notation for $I_{K}$. For a covering $L$ of $K$, we consider the group homomorphism

$$
\iota_{L / K}^{I}: I_{K} \longrightarrow I_{L}, \quad\left(c_{\wp}\right)_{\wp \in S_{K}} \longmapsto\left(\iota_{\mathscr{P} / \iota_{L / K}^{*} \mathscr{P}}^{\times}\left(c_{l_{L / K}^{*} \mathscr{P}}\right)\right) \mathscr{P} \in S_{L} .
$$

Since $S_{L}$ is stable under the action of $\operatorname{Aut}\left(h_{L / K}\right)$, we have a natural action of $\operatorname{Gal}(L / K)$ on $I_{L}$ over $I_{K}$ when $L / K$ is Galois. For any $\left(L_{0}, L_{1}\right) \in M(K)^{2}$ with $L_{0} \leq L_{1}$, regarding $L_{1}$ as an object of $\mathscr{C}_{0} / L_{0}$ through the canonical morphism $\iota_{L_{1} / L_{0}}: L_{0} \rightarrow L_{1}$ in $\mathscr{C}_{0} / \mathrm{K}$ corresponding to the base points of $\mathscr{F}_{0}\left(O_{L_{0}}\right)$ and $\mathscr{F}_{0}\left(O_{L_{1}}\right)$, we obtain $\iota_{L_{1} / L_{0}}^{I} \circ \iota_{L_{0} / K}^{I}=$ $\iota_{L_{1} / K}$. Therefore the correspondence $L \leadsto I_{L}$ gives a functor $\mathscr{C}_{0} / K \rightarrow \mathrm{Ab}$.

Remark 2.8 In the preceding formulation in [NU], a counterpart of the idèle group is given as the restricted product of the first singular homology groups $\mathrm{H}_{1}$. Through the Poincaré duality, it can be interpreted as the restricted product of $\widehat{\mathrm{H}}_{\mathrm{m}}^{1}$. Therefore, our formulation of $I_{K}$ is a refinement of the counterpart of the idèle group in [NU]. The difference is very important because $I_{K}$ satisfies the descent property as will be shown in Theorem 3.12.

It is obvious that $I_{K}$ is the preimage of $\mathbb{Z}^{\oplus S_{K}} \subset \mathbb{Z}^{S_{K}}$ by the group homomorphism $\prod_{\wp \in S_{K}} v_{\wp}: \prod_{\wp \in S_{K}} K_{\wp}^{\times} \rightarrow \mathbb{Z}^{S_{K}},\left(c_{\wp}\right)_{\wp \in S_{K}} \mapsto\left(v_{\wp}\left(c_{\wp}\right)\right)$. Therefore we obtain a natural group homomorphism $v_{K}: I_{K} \rightarrow \mathbb{Z}^{\oplus S_{K}}$. We put $U_{K}:=\operatorname{ker}\left(v_{K}\right)=\prod_{\wp \in S_{K}}\left(O_{K}\right)_{\wp}^{\times} \subset I_{K}$. By the definition of $v_{K}$, we have $\prod_{\wp \in S_{K}} \widehat{\mathrm{B}}_{\mathrm{m}}^{1}\left(K_{\wp}\right) \subset U_{K}$.

By the definition of $K^{\times}=\widehat{Z}_{\mathrm{m}}^{1}\left(S_{K}^{-1} O_{K}\right)$, the image of the composite of the group homomorphisms $\prod_{\wp \in S_{K}} l_{\wp}^{\times}: K^{\times} \rightarrow \prod_{\wp \in S_{K}} K_{\wp}^{\times}, c \mapsto\left(l_{\wp}^{\times}(c)\right)_{\wp \in S_{K}}$ and $\prod_{\wp \in S_{K}} v_{\wp}$ is contained in $\mathbb{Z}^{\oplus S_{K}}$, and hence the image of $\prod_{\wp \in S_{K}} l_{\wp}^{\times}$is contained in $I_{K}$. We obtain a natural group homomorphism $\iota_{K}^{I}: K^{\times} \rightarrow I_{K}$, and denote by $P_{K} \subset I_{K}$ its image. We put $C_{K}:=I_{K} / P_{K}$. Then $P_{K}$ and $C_{K}$ are analogous to the principal idèle group $\mathscr{P}_{k}$ and the idèle class group $\mathscr{C}_{k}$ of $k$. The correspondences $L \leadsto P_{L}, C_{L}$ give functors $\mathscr{C}_{0} / K \rightarrow \mathrm{Ab}$, by definition.

Remark 2.9 Unlike the diagonal embedding, $k^{\times} \rightarrow \mathscr{I}_{k}, \iota_{K}^{I}$ is not injective.

We introduce several topologies. For each $\wp \in S_{K}$, we equip $K_{\wp}^{\times}$the topology generated by the set of subsets of the form $c+U$ for some $c \in K_{\wp}^{\times}$and some subgroup $U \subset K_{\wp}^{\times}$ such that $\left(O_{K}\right)_{\wp}^{\times} \cap U$ is a subgroup of $\left(O_{K}\right)_{\wp}^{\times}$of finite index containing $\widehat{\mathrm{B}}_{\mathrm{m}}^{1}\left(K_{\wp}\right)$, and $\left(O_{K}\right)_{\wp}^{\times}$with the relative topology of $K_{\wp}^{\times}$. We equip $U_{K}$ with the direct product topology of $\left(\left(O_{K}\right)_{\wp}^{\times}\right)_{\wp \in S_{K}}$, and $I_{K}$ with the topology generated by the set of subsets of the form $c+U$ for some $c \in I_{K}$ and some open subgroup $U$ of $U_{K}$. Then $I_{K}$ forms a topological group. We note that $I_{K}$ is not Hausdorff unless $S_{K}=\varnothing$, because every neighbourhood of 0 in $I_{K}$ contains $\prod_{\wp \in S_{K}} \widehat{\mathrm{B}}_{\mathrm{m}}^{1}\left(K_{\wp}\right)$. We equip $C_{K}$ with the quotient topology of $I_{K}$.

\subsection{Analogue of Ray Class Groups}

For a $(\wp, n) \in S_{K} \times \mathbb{N}_{>0}$, we put $U_{\wp}^{(n)}:=\left\{c \in\left(O_{K}\right)_{\wp}^{\times} \mid v_{\wp}^{\log }(c) \in n \mathbb{Z}\right\}$. We denote

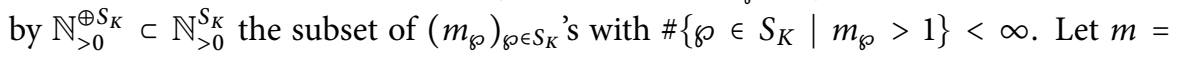


$\left(m_{\wp}\right)_{\wp \in S_{K}} \in \mathbb{N}_{>0}^{\oplus S_{K}}$. We put $I_{K}^{m}:=\prod_{\wp \in S_{K}} U_{\wp}^{\left(m_{\wp}\right)} \subset U_{K}$ and $C_{K}^{m}:=I_{K}^{m} /\left(I_{K}^{m} \cap P_{K}\right)$, and call them the congruence subgroup of $I_{K} \bmod m$ and the ray class group of $K \bmod m$, respectively. Then they are analogous to the congruence subgroup $\mathscr{I}_{k}^{\mathscr{M}} \subset \mathscr{I}_{k}$ and the ray class group $\mathscr{C}_{k}^{\mathscr{M}} \bmod$ non zero ideal $\mathscr{M} \subset \mathscr{O}_{k}$, respectively. Through the inclusion $I_{K}^{m} \rightarrow I_{K}$, we regard $C_{K}^{m}$ as a subgroup of $C_{K}$. We note that $I_{K}^{m}$ contains $\prod_{\wp \in S_{K}} \widehat{\mathrm{B}}_{\mathrm{m}}^{1}\left(K_{\wp}\right)$, and is an open subgroup of $I_{K}$ by definition.

We put $s_{m}:=\left\{\wp \in S_{K} \mid m_{\wp}>1\right\} \in \operatorname{Fin}\left(S_{K}\right)$ and

$$
J_{K}^{m}:=\left\{\left(n_{\wp}\right)_{\wp \in S_{K}} \in \mathbb{Z}^{\oplus S_{K}} \mid \forall \wp \in s_{m}, n_{\wp}=0\right\} .
$$

We denote by $P_{K}^{m} \subset J_{K}^{m}$ the subgroup of $\left(n_{\wp}\right)_{\wp \in S_{K}}$ 's such that there is a $c \in K^{\times}$with $\iota_{\wp}^{\times}(c) \in U_{\wp}^{\left(m_{\wp}\right)}$ for any $\wp \in s_{m}$ and $v_{\wp}\left(l_{\wp}(c)\right)=n_{\wp}$ for any $\wp \in S_{K}$. We put $\mathrm{Cl}_{K}^{m}:=$ $J_{K}^{m} / P_{K}^{m}$. Then $\mathrm{Cl}_{K}^{m}$ is analogous to the generalisation $\mathscr{C} \ell_{k}^{\mathscr{M}}$ of the ideal class group of $k$ [Neu99, §VI 1 p. 365].

Remark 2.10 Put $m_{0}=(1)_{\wp \in S_{K}} \in \mathbb{N}_{>0}^{\oplus S_{K}}$. Then we have $s_{m_{0}}=\varnothing, I_{K}^{m_{0}}=U_{K}$, and $J_{K}^{m_{0}}=\mathbb{Z}^{\oplus S_{K}}$. If $K$ is admissible, then the group homomorphism

$$
J_{K}^{m_{0}} \longrightarrow \mathrm{H}_{1}(M),\left(n_{\wp}\right)_{\wp \in S_{K}} \longmapsto \sum_{\wp \in S_{K}} n_{\wp} \operatorname{Frob}_{\wp}
$$

is surjective, by definition. If $K$ is orientable, then its kernel coincides with $P_{K}^{m_{0}}$ by a standard argument using the Mayer-Vietoris exact sequence and the Poincaré duality (3.1). In particular, if $K$ is orientable and admissible, then we have a natural group isomorphism $\mathrm{Cl}_{K}^{m_{0}} \cong \mathrm{H}_{1}(M)$. Therefore $\mathrm{Cl}_{K}^{m}$ for a general $m \in \mathbb{N}_{>0}^{\oplus S_{K}}$ is a generalisation of $\mathrm{H}_{1}(M)$.

\subsection{Resulting Dictionary}

In Tables 1-4, we collect the analogues into a dictionary that is our variant of the $\mathrm{M}^{2} \mathrm{KR}$ dictionary.

\section{Main Results}

Continuing from Section 2, let $K=\left(O_{K}, S_{K}\right)$ denote an arithmetic manifold with a fixed base point $\iota_{\infty} \in \mathscr{F}_{0}\left(O_{K}\right) \backslash \operatorname{im}\left(S_{K}\right)$. We put $M:=\mathscr{F}_{0}\left(O_{K}\right)$. We establish a class field theory for an arithmetic topology.

\subsection{Galois Theory}

We establish the Galois theory for coverings of arithmetic manifolds. To begin with, we give a criterion for a Galois covering.

Proposition 3.1 A covering $L$ of $K$ is Galois if and only if there is an $s \in \operatorname{Fin}\left(S_{K}\right)$ such that the restriction $\mathscr{F}_{0}\left(O_{L}\right) \backslash \operatorname{im}\left(h_{L / K}^{*}(s)\right) \rightarrow M \backslash \operatorname{im}(s)$ of $h_{L / K}$ is an unbranched Galois covering. 
Table 1

\begin{tabular}{|c|c|}
\hline \multicolumn{2}{|c|}{ Integral Object } \\
\hline $\begin{array}{c}\text { a ring } \mathscr{O} \text { of } \\
\text { algebraic integers }\end{array}$ & $\begin{array}{l}\text { an orientable closed } \\
\text { arithmetic object } O \text { of } \mathscr{C}_{0}\end{array}$ \\
\hline $\begin{array}{l}\text { a surjective homomorphism } \\
\qquad \mathscr{O} \rightarrow \mathbb{F}_{q}\end{array}$ & $\begin{array}{c}\text { a tame cycle } \\
O \rightarrow \mathscr{A}_{\mathrm{S}^{1}}\end{array}$ \\
\hline $\begin{array}{l}\text { the absolute Galois group } \\
\qquad G_{\mathbb{F}_{q}} \cong \widehat{\mathbb{Z}}\end{array}$ & $\begin{array}{l}\text { the fundamental group } \\
\qquad \pi_{1}\left(S^{1}, 1\right)^{\mathrm{op}} \cong \mathbb{Z}\end{array}$ \\
\hline $\begin{array}{c}\text { a maximal ideal } \\
p \text { of } \mathscr{O}\end{array}$ & $\begin{array}{l}\text { a tame knot } \\
\wp \text { in } O\end{array}$ \\
\hline $\begin{array}{l}\text { the cardinality } \\
\qquad \# \mathscr{O} / p\end{array}$ & $\begin{array}{l}\text { the exponential length } \\
\qquad \# / \wp\end{array}$ \\
\hline $\begin{array}{l}\text { the maximal spectrum } \\
\qquad \operatorname{Max}(\mathscr{O})\end{array}$ & $\begin{array}{l}\text { a stably generic link } \\
\qquad S \text { in } O\end{array}$ \\
\hline $\begin{array}{l}\text { the ideal class group } \\
\qquad \mathscr{C} \ell_{\mathrm{Frac}(\mathscr{O})}\end{array}$ & $\begin{array}{l}\text { the profinite completion of } \\
\text { the first homology group } \\
\qquad \mathrm{H}_{1}\left(\mathscr{F}_{0}(O)\right)\end{array}$ \\
\hline
\end{tabular}

Table 2

\begin{tabular}{|c|c||}
\hline \hline \multicolumn{2}{|c||}{ Rational Object } \\
\hline \hline $\begin{array}{c}\text { a number field } \\
\text { an infinite place } \\
i_{\infty}: k \hookrightarrow \mathbb{C}\end{array}$ & $\begin{array}{c}\text { an orientable closed stably generic } \\
\text { arithmetic manifold } K\end{array}$ \\
\hline$k^{\times}$ & $\iota_{\infty} \in \mathscr{F}_{0}\left(O_{K}\right) \backslash \mathrm{im}\left(S_{K}\right)$ \\
\hline the multiplicative group & the multiplicative group \\
the unit group & $K^{\times}$ \\
$\mathscr{O}_{k}^{\times}$ & the unit group \\
$\mathscr{I}_{k}$ & $O_{K}^{\times}$ \\
\hline the idèle group & $I_{K}$ \\
\hline $\mathscr{P}_{k}$ & the idèle group \\
$\mathscr{C}_{k}$ & $P_{K}$ \\
\hline the principal idèle group & the idèle class group \\
$\mathscr{I}_{k}^{\mathscr{M}}$ & $C_{K}$ \\
\hline the congrincipal idèle group \\
$\mathscr{C}_{k}^{\mathscr{M}}$ & $I_{K}^{m}$ \\
\hline the ray group & the congruence subgroup \\
\hline \hline
\end{tabular}


Table 3

\begin{tabular}{|c|c|}
\hline \multicolumn{2}{|c|}{ Relative Object } \\
\hline an extension $l / k$ & a covering $L / K$ \\
\hline unramified & unbranched \\
\hline Galois & Galois \\
\hline Abelian & Abelian \\
\hline $\begin{array}{l}\text { the Galois group } \\
\mathrm{Gal}(l / k)\end{array}$ & $\begin{array}{l}\text { the Galois group } \\
\operatorname{Gal}(L / K)\end{array}$ \\
\hline the algebraic closure & the algebraic closure \\
\hline $\begin{array}{c}\bar{k} \subset \mathbb{C} \text { of } k \text { with respect to } \\
\text { the infinite place } i_{\infty}\end{array}$ & $\begin{array}{l}\bar{K} \text { of } K \text { with respect to } \\
\text { the base point } \iota_{\infty}\end{array}$ \\
\hline the absolute Galois group & the absolute Galois group \\
\hline $\operatorname{Gal}(\bar{k} / k)$ & $G_{K}$ \\
\hline an extension & a covering \\
\hline$l / k$ in $\bar{k}$ & $L$ in $M(K)$ \\
\hline
\end{tabular}

Table 4

\begin{tabular}{|c|c|}
\hline \multicolumn{2}{|c|}{ Local Object } \\
\hline $\begin{array}{l}\text { the completion } k_{p} \\
\text { at a } p \in \operatorname{Max}\left(\mathscr{O}_{k}\right)\end{array}$ & $\begin{array}{l}\text { the formal completion } K_{\wp} \\
\text { at a } \wp \in S_{K}\end{array}$ \\
\hline $\begin{array}{l}\text { the multiplicative group } \\
k_{p}^{\times}\end{array}$ & $\begin{array}{c}\text { the multiplicative group } \\
K_{\wp}^{\times}\end{array}$ \\
\hline $\begin{array}{c}\text { the valuation } \\
v_{p}: k_{p}^{\times} \rightarrow \mathbb{Z}\end{array}$ & $\begin{array}{c}\text { the valuation } \\
v_{\wp}: K_{\wp}^{\times} \rightarrow \mathbb{Z}\end{array}$ \\
\hline $\begin{array}{l}\text { the unit group } \\
\left(\mathscr{O}_{k}\right)_{p}^{\times} \rightarrow \mathbb{Z}\end{array}$ & $\begin{array}{l}\text { the unit group } \\
\left(O_{K}\right)_{\wp}^{\times}\end{array}$ \\
\hline $\begin{array}{l}\text { the decomposition group } \\
\operatorname{Gal}(l / k)_{P}\end{array}$ & $\begin{array}{l}\text { the decomposition group } \\
\operatorname{Gal}(L / K)_{\mathscr{P}}\end{array}$ \\
\hline $\begin{array}{l}\text { the Frobenius } \\
\text { Frob }_{P}\end{array}$ & $\begin{array}{l}\text { the Frobenius } \\
\quad \text { Frob } \mathscr{P}\end{array}$ \\
\hline $\begin{array}{l}\text { a place } \widehat{p} \\
\text { of } \bar{k} \text { over } P\end{array}$ & $\begin{array}{c}\text { a place } \widehat{\wp} \\
\text { of } \bar{K} \text { over } \wp\end{array}$ \\
\hline $\begin{array}{c}\text { the algebraic closure } \\
\bar{k}_{\widehat{p}} \text { of } k_{p} \text { with respect to } \\
\text { the place } \widehat{p}\end{array}$ & $\begin{array}{c}\text { the algebraic closure } \\
\bar{K}_{\widehat{\wp}} \text { of } K \text { with respect to } \\
\text { the place } \widehat{\wp}\end{array}$ \\
\hline
\end{tabular}


Proof By the definition of a Galois covering of $K$, the assertion immediately follows from the fact that the cardinality of every fibre of an unbranched covering of connected locally compact topological spaces is constant.

We give a presentation of the Galois group of a covering of arithmetic manifolds.

Proposition 3.2 Let $s \in \operatorname{Fin}\left(S_{K}\right)$. For any covering $L$ of $K$ unbranched outside $\operatorname{im}(s)$ with $\iota_{\infty}^{\prime} \in h_{L / K}^{-1}\left(\iota_{\infty}\right)$, the following assertions hold.

(i) The action of $\pi_{1}\left(M \backslash \operatorname{im}(s), \iota_{\infty}\right)$ on $\mathscr{F}_{0}\left(O_{L}\right) \backslash \operatorname{im}\left(h_{L / K}^{*}(s)\right)$ over $M \backslash \operatorname{im}(s)$ induces an exact sequence of pointed sets.

$\pi_{1}\left(\mathscr{F}_{0}\left(O_{L}\right) \backslash \operatorname{im}\left(h_{L / K}^{*}(s)\right), \iota_{\infty}^{\prime}\right) \longrightarrow \pi_{1}\left(M \backslash \operatorname{im}(s), \iota_{\infty}\right) \longrightarrow\left(h_{L / K}^{-1}\left(\iota_{\infty}\right), \iota_{\infty}^{\prime}\right) \longrightarrow\{1\}$

(ii) If $L / K$ is Galois, then the exact sequence in (i) yields an exact sequence of groups.

$\pi_{1}\left(\mathscr{F}_{0}\left(O_{L}\right) \backslash \operatorname{im}\left(h_{L / K}^{*}(s)\right), \iota_{\infty}^{\prime}\right)^{\text {op }} \longrightarrow \pi_{1}\left(M \backslash \operatorname{im}(s), \iota_{\infty}\right)^{\text {op }} \longrightarrow \operatorname{Gal}(L / K) \longrightarrow\{1\}$

(iii) If $L / K$ is Abelian, then the exact sequence in (ii) yields an exact sequence of Abelian groups

$\mathrm{H}_{1}\left(\mathscr{F}_{0}\left(O_{L}\right) \backslash \operatorname{im}\left(h_{L / K}^{*}(s)\right)\right) \longrightarrow \mathrm{H}_{1}(M \backslash \operatorname{im}(s)) \longrightarrow \operatorname{Gal}(L / K) \longrightarrow\{1\}$,

independent of $\iota_{\infty}$ and $\iota_{\infty}^{\prime}$ through the Hurewicz isomorphisms.

Proof The first assertion is purely topological and elementary. The restriction of the action of $\mathrm{Gal}(L / K)$ on $\mathscr{F}_{0}\left(O_{L}\right)$ to $\mathscr{F}_{0}\left(O_{L}\right) \backslash \mathrm{im}\left(h_{L / K}^{*}(s)\right)$ is transitive, and hence induces a group isomorphism $\operatorname{Gal}(L / K) \rightarrow \operatorname{Aut}\left(\left.h_{L / K}\right|_{\mathscr{F}_{0}\left(O_{L}\right) \backslash \operatorname{im}\left(h_{L / K}^{*}(s)\right)}\right)^{\text {op }}$. Therefore the other assertions immediately follow from the first assertion.

We call the image of $\pi_{1}\left(\mathscr{F}_{0}\left(O_{L}\right) \backslash \operatorname{im}\left(h_{L / K}^{*}(s)\right), \iota_{\infty}^{\prime}\right)$ in $\pi_{1}(M \backslash \operatorname{im}(s))$ (resp. the image of $\mathrm{H}_{1}\left(\mathscr{F}_{0}\left(O_{L}\right) \backslash \operatorname{im}\left(h_{L / K}^{*}(s)\right)\right)$ in $\left.\mathrm{H}_{1}(M \backslash \operatorname{im}(s))\right)$ by the homomorphism in Proposition 3.2 the subgroup corresponding to $\iota_{L / K}$. As a consequence of Proposition 3.1, we obtain the following.

Corollary 3.3 A covering $L$ of $K$ is Galois if and only if there is an $s_{0} \in \operatorname{Fin}\left(S_{K}\right)$ such that $h_{L / K}$ is unbranched outside $\operatorname{im}\left(s_{0}\right)$ and for any $s_{1} \in \operatorname{Fin}\left(S_{K}\right)$ with $s_{0} \leq s_{1}$, the subgroup of $\pi_{1}\left(\mathscr{F}_{0}\left(O_{K}\left[s_{1}^{-1}\right]\right), \iota_{\infty}\right)$ corresponding to $\iota_{L / K}$ is normal.

Now we construct the Galois correspondence for arithmetic manifolds.

Theorem 3.4 (Galois Theory) The following assertions hold.

(i) For any $L \in M(K)$, there is a unique open subgroup $H_{L / K} \subset G_{K}$ such that for any $\widetilde{L} \in M^{\mathrm{Gal}}(K)$ with $L \leq \widetilde{L}$, the image of $H_{L / K}$ by the canonical projection

$$
G_{K} \longrightarrow \operatorname{Gal}(\widetilde{L} / K)
$$

coincides with $\operatorname{Gal}(\widetilde{L} / L)$.

(ii) For any open subgroup $H \subset G_{K}$, there is a unique $L_{H} \in M(K)$ such that for any $\widetilde{L} \in M^{\mathrm{Gal}}(K)$ with $L_{H} \leq \widetilde{L}$, the image of $H$ by the canonical projection $G_{K} \rightarrow \operatorname{Gal}(\widetilde{L} / K)$ coincides with $\operatorname{Gal}\left(\widetilde{L} / L_{H}\right)$. 
Proof First, let $L \in M(K)$. The uniqueness of $H_{L / K}$ in the assertion immediately follows from the definition of the topology of $G_{K}$. By the cofinality of $M^{\mathrm{Gal}}(K)$ in $M(K)$, there is an $\widetilde{L}_{0} \in M^{\mathrm{Gal}}(K)$ with $L \leq \widetilde{L}_{0}$. We denote by $H_{L / K} \subset G_{K}$ the preimage of $\operatorname{Gal}\left(\widetilde{L}_{0} / L\right)$ by the canonical projection $G_{K} \rightarrow \operatorname{Gal}\left(\widetilde{L}_{0} / K\right)$. By the definition of the topology of $G_{K}, H_{L / K}$ is an open subgroup of $G_{K}$.

Let $\widetilde{L} \in M^{\mathrm{Gal}}(K)$ with $L \leq \widetilde{L}$. Since $M(K)$ is directed and $M^{\mathrm{Gal}}(K)$ is cofinal in $M(K)$, there is an $\widetilde{L}_{1} \in M^{\mathrm{Gal}}(K)$ with $\widetilde{L}_{0} \leq \widetilde{L}_{1}$ and $\widetilde{L} \leq \widetilde{L}_{1}$. For any $\widetilde{L}_{2} \in\left\{\widetilde{L}_{0}, \widetilde{L}\right\}$, the preimage of $\operatorname{Gal}\left(\widetilde{L}_{2} / L\right)$ by the canonical projection $\operatorname{Gal}\left(\widetilde{L}_{1} / K\right) \rightarrow \operatorname{Gal}\left(\widetilde{L}_{2} / K\right)$ coincides with $\operatorname{Gal}\left(\widetilde{L}_{1} / L\right)$ by definition. Therefore the preimage of $\operatorname{Gal}(\widetilde{L} / L)$ by the canonical projection $G_{K} \rightarrow \operatorname{Gal}(\widetilde{L} / K)$ coincides with $H_{L / K}$.

Secondly, let $H \subset G_{K}$ be an open subgroup. The uniqueness of $L_{H}$ in the assertion immediately follows from the cofinality of $M^{\mathrm{Gal}}(K)$ in $M(K)$ and the classical Galois theory for unbranched coverings, because for any $\widetilde{L} \in M(K)$, every automorphism in Top of the antecedent of the unbranched covering associated with $h_{\widetilde{L} / K}$ over $M$ uniquely extends to an automorphism in Top of $\mathscr{F}_{0}\left(O_{\widetilde{L}}\right)$ over $M$. By the definition of the topology of $G_{K}$, there is an $\widetilde{L}_{0} \in M^{\mathrm{Gal}}(K)$ such that $H$ contains the kernel of the canonical projection $G_{K} \rightarrow \operatorname{Gal}\left(\widetilde{L}_{0} / K\right)$. We denote by $L_{H} \in M(K)$ a unique element with $L_{H} \leq \widetilde{L}_{0}$ such that the image of $H$ by the canonical projection

$$
G_{K} \longrightarrow \operatorname{Gal}\left(\widetilde{L}_{0} / K\right)
$$

coincides with $\mathrm{Gal}\left(\widetilde{L} / L_{H}\right)$, which exists because the quotient of the unbranched covering associated with $h_{\widetilde{L} / K}$ by the action of the image of $H$ is an unbranched covering.

Let $\widetilde{L} \in M^{\mathrm{Gal}}(K)$ with $L_{H} \leq \widetilde{L}$. By the argument above, there is an $\widetilde{L}_{1} \in M^{\mathrm{Gal}}(K)$ with $\widetilde{L}_{0} \leq \widetilde{L}_{1}$ and $\widetilde{L} \leq \widetilde{L}_{1}$. For any $\widetilde{L}_{2} \in\left\{\widetilde{L}_{0}, \widetilde{L}\right\}$, the preimage of $\operatorname{Gal}\left(\widetilde{L}_{2} / L_{H}\right)$ by the canonical projection $\operatorname{Gal}\left(\widetilde{L}_{1} / K\right) \rightarrow \operatorname{Gal}\left(\widetilde{L}_{2} / K\right)$ coincides with $\operatorname{Gal}\left(\widetilde{L}_{1} / L_{H}\right)$ by definition. Therefore the preimage of $\operatorname{Gal}\left(\widetilde{L} / L_{H}\right)$ by the canonical projection

$$
G_{K} \longrightarrow \operatorname{Gal}(\widetilde{L} / K)
$$

coincides with $H$.

We fix a $\wp \in S_{K}$ and a place $\widehat{\wp}=\left(\wp_{L}\right)_{L \in M(K)}$ of $\bar{K}$ over $\wp$. As an application of Theorem 3.4 (ii), we obtain the existence of a covering of $K$ whose behaviour around $\widehat{\wp}$ is completely controlled, in the following sense.

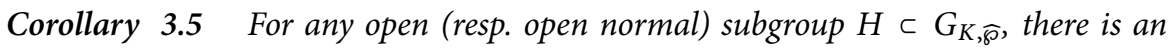
$L \in M(K)$ (resp. $\left.L \in M^{\mathrm{Gal}}(K)\right)$ such that for any $\widetilde{L} \in M^{\mathrm{Gal}}(K)$ with $L \leq \widetilde{L}$, the image of $H$ by the canonical projection $G_{K, \widetilde{\wp}} \rightarrow \operatorname{Gal}(\widetilde{L} / K)_{\wp_{\widetilde{L}}}$ coincides with $\mathrm{Gal}(\widetilde{L} / L)_{\wp_{\widetilde{L}}}$.

We note that we will show in Corollary 3.20 that $G_{K, \widetilde{乃}}$ is in fact Abelian, and hence the assumption of the normality automatically holds.

Proof By an elementary argument on a profinite group, there is an open subgroup $\widetilde{H} \subset G_{K}$ with $\widetilde{H} \cap G_{K, \widetilde{\wp}}=H$. Then $L_{\widetilde{H}} \in M(K)$ satisfies the desired property. 


\subsection{Descent Theory}

We verify several descent properties that allow us to establish class field theory in arithmetic topology in a cocycle-theoretic way. To begin with, we study the descent property of $\mathrm{C}_{\mathrm{m}}^{*}$ with respect to a Galois unbranched morphism $\varphi$ : $O_{0} \rightarrow O_{1}$ in $\mathscr{C}_{0}$. We put $G:=\operatorname{Aut}\left(\mathscr{F}_{0}(\varphi)\right)$. For a left $\mathbb{Z}[G]$-module $A$, we denote by $\left(\widehat{\mathrm{H}}^{n}(G, A)\right)_{n \in \mathbb{Z}}$ the Tate cohomology of $A$, and put $A^{G}:=\{c \in A \mid$ for all $g \in G, g \cdot c=c\}$.

Proposition 3.6 For any $n \in \mathbb{N}$, the group homomorphisms

$$
\begin{aligned}
& \mathrm{C}_{\mathrm{m}}^{n}(\varphi): \mathrm{C}_{\mathrm{m}}^{n}\left(O_{0}\right) \longrightarrow \mathrm{C}_{\mathrm{m}}^{n}\left(O_{1}\right), \\
& \mathrm{Z}_{\mathrm{m}}^{n}(\varphi): \mathrm{Z}_{\mathrm{m}}^{n}\left(O_{0}\right) \longrightarrow \mathrm{Z}_{\mathrm{m}}^{n}\left(O_{1}\right)
\end{aligned}
$$

are isomorphisms onto $\mathrm{C}_{\mathrm{m}}^{n}\left(O_{1}\right)^{G}$ and $\mathrm{Z}_{\mathrm{m}}^{n}\left(O_{1}\right)^{G}$, respectively, with respect to the action of $G$ on $O_{1}$ in $\mathscr{C}_{0}$ introduced in Section 2.3.

Proof Since the action of $G$ on $O_{1}$ induces an action of $G$ on $\mathrm{C}_{\mathrm{m}}^{*}\left(O_{1}\right)$ over $\mathrm{C}_{\mathrm{m}}^{*}\left(O_{0}\right)$, it suffices to verify that $\mathrm{C}_{\mathrm{m}}^{n}(\varphi)$ is a group isomorphism onto $\mathrm{C}_{\mathrm{m}}^{n}\left(O_{1}\right)^{G}$. The inclusion relation $\operatorname{im}\left(\mathrm{C}_{\mathrm{m}}^{n}(\varphi)\right) \subset \mathrm{C}_{\mathrm{m}}^{n}\left(O_{1}\right)^{G}$ immediately follows from the definition of the action of $G$. The map

$$
\operatorname{Hom}_{\mathscr{C}}\left(O_{1}, \mathscr{A}_{\Delta^{n}}\right) \longrightarrow \operatorname{Hom}_{\mathscr{C}}\left(O_{0}, \mathscr{A}_{\Delta^{n}}\right), \quad \tilde{\gamma} \longmapsto \tilde{\gamma} \circ \varphi
$$

coincides with the pull-back of the map

$$
\mathrm{C}\left(\Delta^{n}, \mathscr{F}_{0}\left(O_{1}\right)\right) \longrightarrow \mathrm{C}\left(\Delta^{n}, \mathscr{F}_{0}\left(O_{0}\right)\right), \quad \bar{\gamma} \longmapsto \mathscr{F}_{0}(\varphi) \circ \bar{\gamma},
$$

which is surjective by the covering homotopy property, by the map

$$
\operatorname{Hom}_{\mathscr{C}}\left(O_{0}, \mathscr{A}_{\Delta^{n}}\right) \longrightarrow \mathrm{C}\left(\Delta^{n}, \mathscr{F}_{0}\left(O_{0}\right)\right), \quad \gamma \longmapsto \mathscr{F}(\gamma)
$$

by Remark 2.1, and hence is surjective. Therefore $\mathrm{C}_{\mathrm{m}}^{n}(\varphi)$ is injective.

Let $\widetilde{c} \in \mathrm{C}_{\mathrm{m}}^{n}\left(O_{1}\right)^{G}$ and $\gamma \in \operatorname{Hom}_{\mathscr{C}}\left(O_{0}, \mathscr{A}_{\Delta^{n}}\right)$. We put

$$
\operatorname{Hom}_{\mathscr{C}}\left(O_{1}, \mathscr{A}_{\Delta^{n}}\right) / \gamma:=\left\{\tilde{\gamma} \in \operatorname{Hom}_{\mathscr{C}}\left(O_{1}, \mathscr{A}_{\Delta^{n}}\right) \mid \widetilde{\gamma} \circ \varphi=\gamma\right\} .
$$

Then the map

$$
\begin{aligned}
& \operatorname{Hom}_{\mathscr{C}}\left(O_{1}, \mathscr{A}_{\Delta^{n}}\right) / \gamma \longrightarrow\left\{\bar{\gamma} \in \mathrm{C}\left(\Delta^{n}, \mathscr{F}_{0}\left(O_{1}\right)\right) \mid \mathscr{F}_{0}(\varphi) \circ \bar{\gamma}=\mathscr{F}_{0}(\gamma)\right\}, \\
& \widetilde{\gamma} \longmapsto \mathscr{F}_{0}(\widetilde{\gamma})
\end{aligned}
$$

is bijective again by Remark 2.1, and hence the action of $G$ on $\operatorname{Hom}_{\mathscr{C}}\left(O_{1}, \mathscr{A}_{\Delta^{n}}\right) / \gamma$ is transitive. This implies that the image of $\operatorname{Hom}_{\mathscr{C}}\left(O_{1}, \mathscr{A}_{\Delta^{n}}\right) / \gamma$ by $\widetilde{c}$ consists of a single element $c_{\gamma} \in \mathbb{Z}$. We put $c:=\left(c_{\gamma}\right)_{\gamma \in \operatorname{Hom}_{\mathscr{C}}\left(O_{0}, \mathscr{A}_{\Delta^{n}}\right)} \in \mathrm{C}_{\mathrm{m}}^{n}\left(O_{1}\right)$. Then we have $\mathrm{C}_{\mathrm{m}}^{n}(\varphi)(c)=\widetilde{c}$ by the construction. Thus $\mathrm{C}_{\mathrm{m}}^{n}(\varphi)$ is an isomorphism onto $\mathrm{C}_{\mathrm{m}}^{n}\left(O_{1}\right)^{G}$.

We give an analogue of Hilbert's theorem 90 for the counterpart $C_{m}^{1}$ of the multiplicative group of invertible meromorphic sections.

Theorem 3.7 (Hilbert's theorem 90) The equality $\widehat{\mathrm{H}}^{n}\left(G, \mathrm{C}_{\mathrm{m}}^{m}\left(O_{1}\right)\right)=\{0\}$ holds for any $(m, n) \in \mathbb{N} \times \mathbb{Z}$. 
Proof Since $\varphi$ is Galois and unbranched, $G$ acts freely on each fibre of $\varphi$ and hence on $\operatorname{Hom}_{\mathscr{C}}\left(O_{1}, \mathscr{A}_{\Delta^{n}}\right)$. Therefore, $\mathrm{C}_{\mathrm{m}}^{m}\left(O_{1}\right)$ admits a $\mathbb{Z}[G]$-linear isomorphism to the direct product of copies of the left regular $\mathbb{Z}[G]$-module. Thus the assertion holds.

Let $L$ be a Galois covering of $K$. Since $S_{L}$ is stable under the action of $\mathrm{Gal}(L / K)$, we have a natural action of $\operatorname{Gal}(L / K)$ on $L^{\times}$. We obtain the descent property of $\widehat{Z}_{\mathrm{m}}^{1}$ with respect to a Galois covering of arithmetic manifolds.

Theorem 3.8 For any $n \in \mathbb{N}$, the group homomorphism $\widehat{\mathrm{Z}}_{\mathrm{m}}^{n}\left(S_{K}^{-1} O_{K}\right) \rightarrow \widehat{\mathrm{Z}}_{\mathrm{m}}^{n}\left(S_{L}^{-1} O_{L}\right)$ associated with the natural morphism $S_{K}^{-1} O_{K} \rightarrow S_{L}^{-1} O_{L}$ in $\widehat{\mathscr{C}}_{0}$ is an isomorphism onto $\widehat{\mathrm{Z}}_{\mathrm{m}}^{n}\left(S_{L}^{-1} O_{L}\right)^{\mathrm{Gal}(L / K)}$.

Proof The assertion is reduced to the case where $L / K$ is unbranched, because the set of finite subsets of $S_{K}$ containing the branched locus of $h_{L / K}$ is cofinal in $\operatorname{Fin}\left(S_{K}\right)$. Therefore, the assertion immediately follows from Proposition 3.6 for $\iota_{L / K}: O_{K} \rightarrow O_{L}$.

In particular, the group homomorphism $\iota_{L / K}^{\times}: K^{\times} \rightarrow L^{\times}$in Theorem 3.8 for the case $n=1$ is an isomorphism onto $\left(L^{\times}\right)^{\operatorname{Gal}(L / K)}$. As a consequence, we obtain the following.

Corollary 3.9 The canonical group homomorphism $K^{\times} \rightarrow \bar{K}^{\times}$is an isomorphism onto $\left(\bar{K}^{\times}\right)^{G_{K}}$,

Let $\wp \in S_{K}$ and $\mathscr{P} \in S_{L}$ with $\iota_{L / K}^{*} \mathscr{P}=\wp$. The action of $\operatorname{Gal}(L / K)$ on the set of open subsets of $\mathscr{F}_{0}\left(O_{L}\right)$ induces an action of $\operatorname{Gal}(L / K)_{\mathscr{P}}$ on $\operatorname{Nbh}\left(O_{L}, \operatorname{im}(\mathscr{P})\right)$, and hence actions of $\operatorname{Gal}(L / K)_{\mathscr{P}}$ on $\left(O_{L}\right)_{\mathscr{P}}$ and $L_{\mathscr{P}}$ in $\widehat{\mathscr{C}}_{0}$. We note that this action induces a group isomorphism between $\operatorname{Gal}(L / K)_{\mathscr{P}}$ and the group of automorphisms of $L_{\mathscr{P}}$ over $K_{\wp}$ in $\widehat{\mathscr{C}}_{0}$. We obtain the descent property of $\widehat{Z}_{\mathrm{m}}^{1}$ with respect to the formal completion of a Galois covering of arithmetic manifolds.

Theorem 3.10 The group homomorphism $i_{\mathscr{P} / \wp}^{\times}:=\widehat{Z}_{\mathrm{m}}^{1}\left(\iota_{\mathscr{P} / \wp}\right): K_{\wp}^{\times} \rightarrow L_{\mathscr{P}}^{\times}$is an iso-

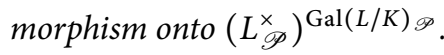

Proof The assertion is reduced to the case

$$
\left.\left(O_{K}, O_{L}, l_{L / K}\right)=\left(\mathscr{A}_{V[r)}, \mathscr{A}_{V\left[r^{e^{-1}}\right.}\right), h_{d, e, f}[r)\right)
$$

for an $(r, d, e, f) \in(0,1] \times \mathbb{Z} \times \mathbb{N}_{>0} \times \mathbb{N}_{>0}$ by the definition of a covering of arithmetic manifolds. Then we have $\operatorname{Gal}(L / K)_{\mathscr{P}}=\mathrm{Gal}(L / K)$ and the group homomor$\operatorname{phism} \operatorname{Gal}(L / K)_{\mathscr{P}} \rightarrow \operatorname{Gal}\left(\left(\left.O_{L}\right|_{V\left(r^{e^{-1}}\right)}, \varnothing\right) /\left(\left.O_{K}\right|_{V(r)}, \varnothing\right)\right)$ given as the restriction is an isomorphism. Therefore, the assertion immediately follows from Proposition 3.6, because $\left(\left.O_{L}\right|_{V\left(r^{-1}\right)}, \varnothing\right) /\left(\left.O_{K}\right|_{V(r)}, \varnothing\right)$ is unbranched. ing.

Let $\widehat{\wp}=\left(\wp_{L}\right)_{L \in M(K)}$ be a place of $\bar{K}$ over $\wp$. By Theorem 3.10, we obtain the follow- 
Corollary 3.11 The canonical group homomorphism $K_{\wp}^{\times} \rightarrow \bar{K}_{\widehat{\wp}}^{\times}$is an isomorphism onto $\left(\bar{K}_{\widehat{\wp}}^{\times}\right)^{G_{K, \widehat{\wp}}}$,

Finally, we obtain the descent property of $\iota_{L / K}^{I}$.

Theorem 3.12 The group homomorphism $\iota_{L / K}^{I}$ is an isomorphism onto $I_{L}^{\mathrm{Gal}(L / K)}$.

Proof By Proposition 3.10, $\iota_{L / K}^{I}$ is injective. By the definition of the action of

$$
\operatorname{Gal}(L / K)
$$

the image of $\iota_{L / K}^{I}$ is contained in $I_{L}^{\mathrm{Gal}(L / K)}$. Let $\widetilde{c}=(\widetilde{c} \mathscr{P}) \mathscr{P}_{\in} \in S_{L} \in I_{L}^{\mathrm{Gal}(L / K)}$. We construct a $c=\left(c_{\wp}\right)_{\wp \in S_{K}}$ with $\iota_{L / K}^{I}(c)=\widetilde{c}$. Let $\wp \in S_{K}$. By Proposition 3.10, for any $\mathscr{P} \in S_{L}$ with $\iota_{L / K}^{*} \mathscr{P}=\wp$, there is a unique $c_{\mathscr{P}} \in K_{\wp}^{\times}$with $\iota_{\mathscr{P} / \wp}^{\times}\left(c_{\mathscr{P}}\right)=\widetilde{c}_{\mathscr{P}}$.

Let $\left(\mathscr{P}_{0}, \mathscr{P}_{1}\right) \in S_{L}^{2}$ with $\iota_{L / K}^{*} \mathscr{P}_{0}=\iota_{L / K}^{*} \mathscr{P}_{1}=\wp$. By the transitivity of the action of $\operatorname{Gal}(L / K)$ on $U \backslash \operatorname{im}\left(h_{L / K}^{*}(\{\wp\})\right)$ for a sufficiently small $\mathrm{Gal}(L / K)$-stable neighbourhood $U$ of $\operatorname{im}\left(h_{L / K}^{*}(\{\wp\})\right)$ given as the disjoint union of tubular neighbourhoods, $\operatorname{Gal}(L / K)$ transitively acts on $h_{L / K}^{*}(\{\wp\})$. Therefore, there is a $g \in \operatorname{Gal}(L / K)$ with $g \cdot \mathscr{P}_{0}=\mathscr{P}_{1}$. We have $\iota_{\mathscr{P}_{1} / \wp}\left(c_{\mathscr{P}_{0}}\right)=\iota_{g \cdot \mathscr{P}_{0} / \wp}\left(c_{\mathscr{P}_{0}}\right)=g \cdot \iota_{\mathscr{P}_{0} / \wp}\left(c_{\mathscr{P}_{0}}\right)=g \cdot \widetilde{c}_{\mathscr{P}_{0}}=\widetilde{c}_{\mathscr{P}_{1}}$, and hence $c \mathscr{P}_{0}=\mathcal{c} \mathscr{P}_{1}$ by the uniqueness of $c \mathscr{P}_{1}$. We denote by $c_{\wp}$ the unique element of the singleton $\left\{c_{\mathscr{P}} \mid \mathscr{P} \in h_{L / K}^{*}(\{\wp\})\right\}$. Then $c:=\left(c_{\wp}\right)_{\wp \in S_{K}}$ satisfies $\iota_{L / K}^{I}(c)=\widetilde{c}$ by the construction.

Remark 3.13 By Remark 2.9, the descent property for $C_{K}$ does not follow from Proposition 3.6, Theorem 3.8, and Theorem 3.12. As a result, we do not expect that global class field theory in arithmetic topology can be formulated in terms of abstract class field theory in the sense of [Neu99]. However, we will verify in Theorem 3.16 that at least local class field theory in arithmetic topology can be formulated in terms of abstract class field theory.

\subsection{Local Class Field Theory}

We establish local class field theory in arithmetic topology. Before that, we compare $\widehat{\mathrm{H}}_{\mathrm{m}}^{1}$ and $\mathrm{H}^{1}$.

\section{Theorem 3.14}

(i) The group homomorphism $\lim _{s \in \operatorname{Fin}\left(S_{K}\right)} \mathrm{H}^{1}(M \backslash \mathrm{im}(s)) \rightarrow \widehat{\mathrm{H}}_{\mathrm{m}}^{1}\left(S_{K}^{-1} O_{K}\right)$ given as the inductive limit of $\left(\Theta_{O_{K}\left[s^{-1}\right]}\right)_{s \in \operatorname{Fin}\left(S_{K}\right)}$ is an isomorphism.

(ii) For any $\wp \in S_{K}$, the group homomorphism $K_{\wp}^{\times} \rightarrow \mathbb{Z}^{2}, c \mapsto\left(v_{\wp}(c), v_{\wp}^{\log }(c)\right)$ induces a group isomorphism $\widehat{\mathrm{H}}_{\mathrm{m}}^{1}\left(K_{\wp}\right) \rightarrow \mathbb{Z}^{2}$.

Proof The first assertion follows from Proposition 2.4 applied to $O_{K}\left[s^{-1}\right]$ for all sufficiently large $s \in \operatorname{Fin}\left(S_{K}\right)$. We prove the second assertion. By the tameness of $\wp$, we can assume that $K$ is presented as $\left(\mathscr{A}_{V[r},\left\{p_{r}\right\}\right)$ for some $r \in(0,1]$, where $p_{r}$ is the tame knot in $\mathscr{A}_{V[r)}$ represented by the morphism $\mathscr{A}_{V[r]} \rightarrow \mathscr{A}_{\Delta^{1}}$ in $\mathscr{C}$ given by 
the $\mathscr{C}$-morphism $\lambda_{r}: \Delta^{1} \rightarrow V[r),\left(t_{0}, t_{1}\right) \mapsto\left(\theta\left(t_{1}\right), 0\right)$. By Proposition 2.4 applied to $V(r)$, it suffices to verify that the canonical group homomorphism

$$
\mathrm{H}_{\mathrm{m}}^{1}\left(\mathscr{A}_{V(r)}\right) \longrightarrow \widehat{\mathrm{H}}_{\mathrm{m}}^{1}\left(\operatorname{Frac}\left(\left(\mathscr{A}_{V[r)}\right)_{\mathrm{S}^{1} \times\{0\}}\right)\right)
$$

is surjective. It is reduced to the surjectivity of the group homomorphism

$$
\mathrm{H}_{\mathrm{m}}^{1}\left(\mathscr{A}_{V(r)}\right) \rightarrow \mathrm{H}_{\mathrm{m}}^{1}\left(\mathscr{A}_{V\left(r^{\prime}\right)}\right)
$$

induced by the inclusion $V\left(r^{\prime}\right) \hookrightarrow V(r)$ for any $r^{\prime} \in(0, r]$ that follows from Proposition 2.4 applied to $V(r)$ and $V\left(r^{\prime}\right)$.

Let $L \in \mathrm{ob}\left(\mathscr{C}_{0} / K\right)$ and $\wp \in S_{K}$. By the cofinality of $M^{\mathrm{Gal}}(K)$ in $M(K)$, there is an $\widetilde{L} \in M^{\mathrm{Gal}}(K)$ such that $\iota_{\widetilde{L} / K}$ factors through $\iota_{L / K}$. We fix an $\iota_{\widetilde{L} / L} \in \operatorname{Hom}_{\mathscr{C}_{0} / K}(L, \widetilde{L})$. Let $\mathscr{P} \in S_{L}$ with $\iota_{L / K}^{*} \mathscr{P}=\wp$ and $\widetilde{P} \in S_{\widetilde{L}}$ with $\iota_{\widetilde{L} / L}^{*} \widetilde{\mathscr{P}}=\mathscr{P}$. Then $\widetilde{L} / L$ is Galois and

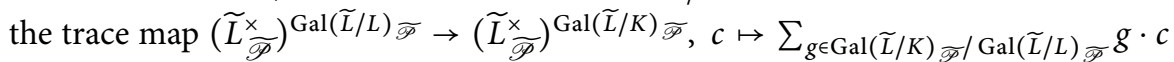
induces a group homomorphism $\operatorname{tr}_{L / K, \mathscr{P}}: L_{\mathscr{P}}^{\times} \rightarrow K_{\wp}^{\times}$that is independent of the choice of $\widetilde{L}$ and $\widetilde{P}$ by Theorem 3.10. We put $N_{L / K, \mathscr{P}}:=\operatorname{im}\left(\operatorname{tr}_{L / K, \mathscr{P}}\right) \subset K_{\wp}^{\times}$.

Suppose that $L / K$ is Galois. We put $\operatorname{Gal}(L / K)_{\wp}:=\bigcap_{\mathscr{P} \in h_{L / K}^{*}(\{\wp\})} \operatorname{Gal}(L / K) \mathscr{P}$. We note that if $L / K$ is Abelian, then $\operatorname{Gal}(L / K) \mathscr{P} \subset \operatorname{Gal}(L / K)$ with $\mathscr{P} \in h_{L / K}^{*}(\{\wp\})$ depends only on $\wp$ by the argument in the first paragraph of Section 2.8 , and hence coincides with $\operatorname{Gal}(L / K)_{\wp}$.

Let $U \in \operatorname{Nbh}\left(O_{K}, \operatorname{im}(\wp)\right)$. Suppose that $U$ is sufficiently small so that $h_{L / K}$ is unbranched at any point of $U \backslash \operatorname{im}(\wp)$. Let $j \in \operatorname{Hom}_{\mathscr{C}}\left(O_{K}, \mathscr{A}_{V[r)}\right)$ be a tubular neighbourhood of $\wp$ with $r \in(0,1]$ and $\operatorname{im}(\mathscr{F}(j)) \subset U$. Then the pullback in Top of $h_{L / K}$ by $\mathscr{F}(j)$ is the disjoint union of branched coverings of $V[r)$ unbranched outside $S^{1} \times\{0\}$ of finite degree. We obtain a group homomorphism

$$
\mathrm{H}_{1}(V(r)) \longrightarrow \pi_{1}\left(M \backslash \operatorname{im}(s), \mathscr{F}(j)\left(1,2^{-1} r\right)\right)
$$

through the Hurewicz isomorphism

$$
\mathrm{H}_{1}(V(r)) \cong \pi_{1}\left(V(r),\left(1,2^{-1} r\right)\right)^{\mathrm{ab}} \cong \pi_{1}\left(V(r),\left(1,2^{-1} r\right)\right),
$$

and hence an action of $\mathrm{H}_{1}(V(r))$ on $\mathscr{F}_{0}\left(O_{L}\right) \backslash \operatorname{im}\left(h_{L / K}^{*}(s)\right)$ over $M \backslash \operatorname{im}(s)$ by $h_{L / K}^{-1}(\operatorname{im}(s))=\operatorname{im}\left(h_{L / K}^{*}(s)\right)$. It induces a group homomorphism

$$
\rho_{j, \wp}: \mathrm{H}_{1}(V(r)) \longrightarrow \mathrm{Gal}(L / K)_{\wp}
$$

by the definition of the action of the meridian and the longitude of $\wp$. It is remarkable that one does not need to $\operatorname{Abelianise} \operatorname{Gal}(L / K)_{\wp}$ here, because $\pi_{1}\left(V(r),\left(1,2^{-1} r\right)\right)$ is Abelian.

We denote by $\rho_{U, \wp}: Z_{\mathrm{m}}^{1}\left(\left.O_{K}\right|_{U \backslash \operatorname{im}(\wp)}\right) \rightarrow \operatorname{Gal}(L / K)_{\wp}$ the composite of the group homomorphism $\mathrm{Z}_{\mathrm{m}}^{1}\left(\left.O_{K}\right|_{U \backslash \mathrm{im}(\wp)}\right) \rightarrow \mathrm{Z}_{\mathrm{m}}^{1}\left(\mathscr{A}_{V(r)}\right)$ associated with the restriction of $\mathscr{F}(j)$, the group homomorphism

$$
\mathrm{Z}_{\mathrm{m}}^{1}\left(\mathscr{A}_{V(r)}\right) \longrightarrow \mathrm{H}_{1}(V(r)), c \longmapsto c\left(\lambda_{r, 2^{-1} r}\right)\left[\mu_{r, 2^{-1} r}\right]-c\left(\mu_{r, 2^{-1} r}\right)\left[\lambda_{r, 2^{-1} r}\right],
$$

and $\rho_{j, \wp}$. Then $\rho_{U, \wp}$ is independent of the choice of $j$ by Proposition 2.6. We denote by $\operatorname{nrs}_{L / K, \wp}: K_{\wp}^{\times} \rightarrow \operatorname{Gal}(L / K)_{\wp}$ the group homomorphism given by the compatible system of group homomorphisms $\rho_{U, \wp}$. Then $\mathrm{nrs}_{L / K, \wp}$ is analogous to the norm residue symbol in local class field theory in number theory. 
We fix a place $\widehat{\wp}=\left(\wp_{L}\right)_{L \in M(K)}$ of $\bar{K}$ over $\wp$. We give a local class field theory in arithmetic topology.

Theorem 3.15 (Local class field theory)

(i) For any $L \in M^{\mathrm{Gal}}(K), N_{L / K, \wp_{L}}$ is an open subgroup of $K_{\wp}^{\times}$of finite index, and $\mathrm{nrs}_{L / K, \wp}$ induces a group isomorphism $K_{\wp}^{\times} / N_{L / K, \wp_{L}} \rightarrow \operatorname{Gal}(L / K)_{\wp_{L}}$.

(ii) Suppose that for any $n \in \mathbb{N}_{>0}$, there is an $L \in M^{\mathrm{Gal}}(K)$ with $n \mid f_{\wp_{L} / \wp}$. Then for any open subgroup $N \subset K_{\wp}^{\times}$of finite index, there is an $L \in M^{\mathrm{Gal}}(K)$ with $N=N_{L / K, \wp_{L}}$.

As is mentioned in Section 2.8, the condition in Theorem 3.15 (ii) holds when $K$ is presented as $\left(\mathscr{A}_{V[r},\left\{p_{r}\right\}\right)$ for some $r \in(0,1]$, where $p_{r}$ is the tame knot introduced in the proof of Theorem 3.14. Therefore, Theorem 3.15 (ii) is an extension of the local class field theory in [NU]. In order to verify Theorem 3.15, we prove the class field axiom in the sense of [Neu99, §IV 6].

Theorem 3.16 (Class field axiom) Suppose that for any $n \in \mathbb{N}_{>0}$, there is an $L \in$

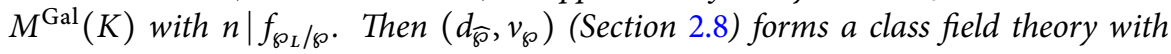
respect to $\left(G_{K, \widehat{\wp}}, \bar{K}_{\widehat{\wp}}^{\times}\right)$.

In order to prove Theorem 3.16, we prepare a lemma. For an $n \in \mathbb{N}_{>0}$, let $\mu_{n} \subset \mathbb{C}^{\times}$ denote the subgroup of $n$-th roots of unity, $\zeta_{n} \in \mu_{n}$ the generator $E\left(n^{-1}\right)$, and $A_{n}$ the $\mathbb{Z}\left[\mu_{n}\right]$-module whose underlying Abelian group is $\widehat{Z}_{\mathrm{m}}^{1}\left(\mathbb{T}^{2}\right)$ and on which the action of $\mu_{n}$ is associated with the right actions $V(r) \times \mu_{n} \rightarrow V(r),((z, w), \zeta) \mapsto(z, w) \cdot[\zeta]:=$ $(z, \zeta w)$ with $r \in(0,1]$. Here we use the bracket $[\cdot]$ in order to emphasise that actions of $\mu_{n}$ on sets, topological spaces, and Abelian groups appearing in this paper are not the scalar multiplication of $\mu_{n}$ on $\mathbb{C}$-vector spaces.

Lemma 3.17 For any $n \in \mathbb{N}_{>0}$, there is a natural group isomorphism $\widehat{\mathrm{H}}^{0}\left(\mu_{n}, A_{n}\right) \cong$ $\mathbb{Z} / n \mathbb{Z}$ and the equality $\widehat{\mathrm{H}}^{1}\left(\mu_{n}, A_{n}\right)=\{0\}$ holds.

Proof First we show $\widehat{\mathrm{H}}^{0}\left(\mu_{n}, A_{n}\right) \cong \mathbb{Z} / n \mathbb{Z}$. We denote by $\operatorname{tr}$ the trace map

$$
A_{n} \longrightarrow A_{n}^{\mu_{n}}, c \longmapsto \sum_{\zeta \in \mu_{n}}[\zeta] \cdot c
$$

For an $r \in(0,1]$, we denote by $A_{n, r}$ the $\mathbb{Z}\left[\mu_{n}\right]$-module whose underlying Abelian group is $\mathrm{Z}_{\mathrm{m}}^{1}(V(r))$ and on which the action of $\mu_{n}$ is associated with the right action $V(r) \times \mu_{n} \rightarrow V(r),((z, w), \zeta) \mapsto(z, w) \cdot[\zeta]$. We also consider the induced actions of $\mu_{n}$ on other objects. By Theorem 3.10 applied to $\mathscr{A}_{h_{0,1, n}\left[r^{n^{-1}}\right)}, h_{0,1, n}\left[r^{n^{-1}}\right)$ induces a group isomorphism $\tau: A_{n} \rightarrow A_{n}^{\mu_{n}}$. Then $\lambda^{\vee} \circ \tau^{-1}: A_{n}^{\mu_{n}, 1, n} \rightarrow \mathbb{Z}$ is surjective. We show $\left(\lambda^{\vee} \circ \tau^{-1}\right)^{-1}(n \mathbb{Z})=\operatorname{im}(\operatorname{tr})$. 
To begin with, let $c \in \operatorname{im}(\operatorname{tr})$. We take a $c_{0} \in A_{n, r}$ with $r \in(0,1]$ such that the image $c_{1}$ of $c_{0}$ in $A_{n}$ satisfies $\operatorname{tr}\left(c_{1}\right)=c$. Then we have

$$
\begin{aligned}
\left(\lambda^{\vee} \circ \tau^{-1}\right)(c) & =\left(\lambda^{\vee} \circ \tau^{-1}\right)\left(\sum_{\zeta \in \mu_{n}}[\zeta] \cdot c_{1}\right)=\sum_{j=0}^{n-1} \sum_{i=0}^{n-1} c_{0}\left(\left[\mathscr{A}_{\left(E\left(n^{-1}\left(t_{1}+j\right)\right), 2^{-1} r \zeta_{n}^{i}\right)}\right]\right) \\
& =n c_{0}\left(\left[\lambda_{r, 2^{-1} r}\right]\right)=n \lambda^{\vee}\left(c_{0}\right) \in n \mathbb{Z}
\end{aligned}
$$

by Proposition 2.4 applied to $V(r)$, because

$$
\left(\sum_{j=0}^{n-1}\left[\left(E\left(n^{-1}\left(t_{1}+j\right)\right), 2^{-1} r \zeta_{n}^{i}\right)\right]\right)-\left[\mathscr{F}\left(\lambda_{r, 2^{-{ }^{-}} r}\right)\right] \in \mathbb{Z}^{\oplus \mathrm{C}\left(\Delta^{1}, V(r)\right)}
$$

is a singular 1-boundary for any $i \in \mathbb{N} \cap[0, n-1]$. It implies $\operatorname{im}(\operatorname{tr}) \subset\left(\lambda^{\vee} \circ \tau^{-1}\right)^{-1}(n \mathbb{Z})$.

Conversely, let $c \in\left(\lambda^{\vee} \circ \tau^{-1}\right)^{-1}(n \mathbb{Z})$. We take a $c_{0} \in A_{n, r}$ with $r \in(0,1]$ such that the image of $c_{0}$ in $A_{n}$ is $c$. Then we have

$$
\begin{aligned}
\mu^{\vee}(c) & =c_{0}\left(\left[\mu_{r, 2^{-1} r}\right]\right)=\sum_{i=0}^{n-1} c_{0}\left(\left[\mathscr{A}_{\left(1,2^{-1} r E\left(n^{-1}\left(t_{1}+i\right)\right)\right)}\right]\right) \\
& =\sum_{\zeta \in \mu_{n}}[\zeta] \cdot c_{0}\left(\left[\mathscr{A}_{\left(1,2^{-1} r E\left(n^{-1} t_{1}\right)\right)}\right]\right)=n c_{0}\left(\left[\mathscr{A}_{\left(1,2^{-1} r E\left(n^{-1} t_{1}\right)\right)}\right]\right) \in n \mathbb{Z},
\end{aligned}
$$

by $c \in A_{n}^{\mu_{n}}$ and Proposition 2.4 applied to $V(r)$, because

$$
\left(\sum_{i=0}^{n-1}\left[\left(1,2^{-1} r E\left(n^{-1}\left(t_{1}+i\right)\right)\right)\right]\right)-\left[\mathscr{F}\left(\mu_{r, 2^{-1} r}\right)\right] \in \mathbb{Z}^{\oplus \mathrm{C}\left(\Delta^{1}, V(r)\right)}
$$

is a singular 1-boundary. We also have

$$
\lambda^{\vee}(c)=c_{0}\left(\left[\lambda_{r, 2^{-1} r}\right]\right)=c_{0}\left(\left[\lambda_{r^{n^{-1}},\left(2^{-1} r\right)^{n^{-1}}} \circ \mathscr{A}_{h_{0,1, n}[r)}\right]\right)=\left(\lambda^{\vee} \circ \tau^{-1}\right)(c) \in n \mathbb{Z},
$$

by $c \in\left(\lambda^{\vee} \circ \tau^{-1}\right)^{-1}(n \mathbb{Z})$ and Proposition 2.4 applied to $V(r)$.

We follow the convention in the proof of Theorem 3.14. By Theorem 3.14 (ii) applied to $\left(V[r),\left\{p_{r}\right\}\right)$, there is a $c^{\prime} \in \widehat{Z}_{\mathrm{m}}^{1}\left(\mathbb{T}^{2}\right)$ with

$$
\left(n \mu^{\vee}\left(c^{\prime}\right), n \lambda^{\vee}\left(c^{\prime}\right)\right)=\left(\mu^{\vee}(c), \lambda^{\vee}(c)\right) .
$$

Then we have $\left(\mu^{\vee}\left(c-\operatorname{tr}\left(c^{\prime}\right)\right), \lambda^{\vee}\left(c-\operatorname{tr}\left(c^{\prime}\right)\right)\right)=(0,0)$, and hence $c-\operatorname{tr}\left(c^{\prime}\right) \in \widehat{\mathrm{B}}_{\mathrm{m}}^{1}\left(\mathbb{T}^{2}\right)$ by Theorem 3.14 (ii). We take a $\Phi \in \widehat{\mathrm{C}}_{\mathrm{m}}^{0}\left(\mathbb{T}^{2}\right)$ with $d^{0} \Phi=c-\operatorname{tr}\left(c^{\prime}\right)$. Replacing $r$ by a sufficiently smaller one, we may assume that $\Phi$ is represented by an $F_{0} \in \mathrm{C}_{\mathrm{m}}^{0}\left(\mathscr{A}_{V(r)}\right)$ with $d^{0} F_{0} \in A_{n, r}^{\mu_{n}}$ by $d^{0} \Phi=c-\operatorname{tr}\left(c^{\prime}\right) \in A_{n}^{\mu_{n}}$ and the finiteness of $\mu_{n}$. Then we have $F_{0} \in \mathrm{C}_{\mathrm{m}}^{0}\left(\mathscr{A}_{V(r)}\right)^{\mu_{n}}$ by $\widehat{\mathrm{H}}^{1}\left(\mu_{n}, \operatorname{ker}\left(d^{0} \mid A_{n, r}\right)\right) \cong \widehat{\mathrm{H}}^{1}\left(\mu_{n}, \mathbb{Z}\right) \cong \operatorname{Hom}_{\mathrm{Ab}}\left(\mu_{n}, \mathbb{Z}\right)=\{0\}$. By Theorem 3.7 applied to $\mathscr{A}_{h_{0,1, n}[r)}$, there is an $F_{1} \in \mathrm{C}_{\mathrm{m}}^{0}\left(\mathscr{A}_{V(r)}\right)$ with $\sum_{\zeta \in \mu_{n}}[\zeta] \cdot F_{1}=$ $F_{0}$. We denote by $c^{\prime \prime} \in \widehat{Z}_{\mathrm{m}}^{1}\left(\mathbb{T}^{2}\right)$ the image of $d^{0} F_{1} \in A_{n, r}$. We obtain $c=\operatorname{tr}\left(c^{\prime}\right)+d^{0} \Phi=$ $\operatorname{tr}\left(c^{\prime}+c^{\prime \prime}\right) \in \operatorname{im}(\operatorname{tr})$. It implies $\left(\lambda^{\vee} \circ \tau^{-1}\right)^{-1}(n \mathbb{Z}) \subset \operatorname{im}(\operatorname{tr})$. Therefore $\widehat{\mathrm{H}}^{0}\left(\mu_{n}, A_{n}\right)$ is isomorphic to $\mathbb{Z} / n \mathbb{Z}$.

Next we show $\widehat{\mathrm{H}}^{1}\left(\mu_{n}, A_{n}\right)=\{0\}$. Since $A_{n}$ is the filtered colimit of $\left(A_{n, r}\right)_{r \in(0,1]}$ in the category of $\mathbb{Z}\left[\mu_{n}\right]$-modules and $\mathbb{Z}\left[\mu_{n}\right]$-linear homomorphisms, it suffices to verify $\widehat{\mathrm{H}}^{1}\left(\mu_{n}, A_{n, r}\right)=\{0\}$ for any $r \in(0,1]$. If $n=1$, then the assertion is obvious. Therefore, we assume $n>1$. 
We prepare the notation. We take a $\left.c_{\lambda} \in \mathrm{Z}_{\mathrm{m}}^{1}(V(r))\right)$ with $c_{\lambda}\left(\lambda_{r, 2^{-{ }^{-1}} r}\right)=1$ that actually exists by Proposition 2.4 applied to $V(r)$. We put

$$
\Sigma:=\left\{\left(z, r^{\prime} E(t)\right) \mid\left(z, r^{\prime}, t\right) \in S^{1} \times(0, r) \times\left[0, n^{-1}\right)\right\} .
$$

Then we have $\left(1,2 r^{-1}\right) \in \Sigma$ and $\left(1,2 r^{-1} \zeta_{n}\right) \notin \Sigma$ by $n>1$, and the map

$$
\Sigma \times \mu_{n} \longrightarrow V(r),((z, w), \zeta) \longmapsto(z, w) \cdot[\zeta]
$$

is bijective. For a $(z, w) \in V(r)$, we denote by $i_{(z, w)} \in \mathbb{N} \cap[0, n-1]$ the unique element with $\left(z, \zeta_{n}^{-i_{(z, w)}} w\right) \in \Sigma$. We put $\Sigma^{\prime}:=\left(\Sigma \backslash\left\{\left(1,2^{-1} r\right)\right\}\right) \sqcup\left\{\left(1,2^{-1} r \zeta_{n}\right)\right\}$. We fix an $\left(\ell_{(z, w)}\right)_{(z, w) \in \Sigma^{\prime}} \in \operatorname{Hom}_{\mathscr{C}}\left(\mathscr{A}_{V(r)}, \mathscr{A}_{\Delta^{1}}\right)^{\Sigma^{\prime}}$ with

$$
\partial\left[\ell_{(z, w)}\right]=[(z, w)]-\left[\left(1,2^{-1} r\right)\right] \in \mathbb{Z}^{\oplus V(r)} \cong \mathbb{Z}^{\oplus \operatorname{Hom}_{\mathscr{C}}\left(\mathscr{A}_{V(r)}, \mathscr{A}_{\Delta^{0}}\right)}
$$

for any $(z, w) \in \Sigma^{\prime}$ that actually exists by the proof of Proposition 2.4 using Whitney's approximation theorem. For each $(z, w) \in V(r)$, we put

$$
L_{(z, w)}:=\left[\ell_{\left(z, \zeta_{n}^{-i(z, w)} w\right)}\right] \cdot\left[\zeta_{n}^{i_{(z, w)}}\right]+\sum_{i=0}^{i_{(z, w)}-1}\left[\ell_{\left(1,2^{-1} r \zeta_{n}\right)}\right] \cdot\left[\zeta_{n}^{i}\right] \in \mathbb{Z}^{\oplus \operatorname{Hom}_{\mathscr{C}}\left(\mathscr{A}_{V(r)}, \mathscr{A}_{\Delta^{1}}\right)}
$$

when $(z, w) \notin\left\{\left(1,2^{-1} r \zeta_{n}^{i}\right) \mid i \in \mathbb{N} \cap[0, n-1]\right\}$ and

$$
L_{(z, w)}:=\sum_{i=0}^{i_{(z, w)}-1}\left[\ell_{\left(1,2^{-1} r \zeta_{n}\right)}\right] \cdot\left[\zeta_{n}^{i}\right] \in \mathbb{Z}^{\oplus \operatorname{Hom}_{\mathscr{C}}\left(\mathscr{A}_{V(r)}, \mathscr{A}_{\Delta^{1}}\right)}
$$

when $(z, w) \in\left\{\left(1,2^{-1} r \zeta_{n}^{i}\right) \mid i \in \mathbb{N} \cap[0, n-1]\right\}$. Then we have

$$
\partial L_{(z, w)}=[(z, w)]-\left[\left(1,2^{-1} r\right)\right]
$$

for any $(z, w) \in V(r)$ by definition.

Let $\chi: \mu_{n} \rightarrow A_{n, r}$ be a 1-cocycle. We put $c_{0}:=\chi\left(\zeta_{n}\right)$. Then we have $\chi\left(\zeta_{n}^{j}\right)=$ $\sum_{i=0}^{j-1}\left[\zeta_{n}^{i}\right] \cdot c_{0}$ for any $j \in \mathbb{N}$, and hence $0=\chi\left(\zeta_{n}^{0}\right)=\chi\left(\zeta_{n}^{n}\right)=\sum_{i=0}^{n-1}\left[\zeta_{n}^{i}\right] \cdot c_{0}$. We set

$$
\begin{aligned}
F & =(F(z, w))_{(z, w) \in V(r)}:=\left(\sum_{i=1}^{i_{(z, w)}} c_{0}\left(L_{\left(z, \zeta_{n}^{-i} w\right)}\right)\right)_{(z, w) \in V(r)} \in \mathbb{Z}^{V(r)} \cong \mathbb{Z}^{\mathrm{C}\left(\Delta^{0}, V(r)\right)} \\
& =\mathbb{Z}^{\operatorname{Hom}_{\mathscr{C}}\left(\mathscr{A}_{V(r)} \cdot \mathscr{A}_{\Delta^{0}}\right)}=\mathrm{C}_{\mathrm{m}}^{0}\left(\mathscr{A}_{V(r)}\right) .
\end{aligned}
$$

Then we have $F(z, w)=0$ for any $(z, w) \in \sum$ and $F(z, w)=F\left(z, \zeta_{n}^{-1} w\right)+c_{0}\left(L_{\left(z, \zeta_{n}^{-1} w\right)}\right)$ for any $(z, w)) \in V(r) \backslash \Sigma$. By the construction, we obtain

$$
\begin{aligned}
F(z, w) & =\sum_{i=1}^{i_{(z, w)}} c_{0}\left(L_{\left(z, \zeta_{n}^{-i} w\right)}\right) \\
& =\sum_{i=1}^{i_{(z, w)}} c_{0}\left(\left[\ell_{\left(z, \zeta_{n}^{-i(z, w)} w\right)}\right] \cdot\left[\zeta_{n}^{i_{(z, w)}-i}\right]+\sum_{j=0}^{i_{(z, w)}-i-1}\left[\ell_{\left(1,2^{-1} r \zeta_{n}\right)}\right] \cdot\left[\zeta_{n}^{j}\right]\right) \\
& \left.=\sum_{i=0}^{i_{(z, w)}-1} c_{0}\left(\left[\ell_{\left(z, \zeta_{n}^{-i}(z, w)\right.}\right)\right] \cdot\left[\zeta_{n}^{i}\right]+\sum_{j=0}^{i-1}\left[\ell_{\left(1,2^{-1} r \zeta_{n}\right)}\right] \cdot\left[\zeta_{n}^{j}\right]\right) \\
& =\sum_{i=0}^{i_{(z, w)}-1} c_{0}\left(\left[\ell_{\left(z, \zeta_{n}^{-i(z, w)} w\right)}\right] \cdot\left[\zeta_{n}^{i}\right]+\left(i_{(z, w)}-1-i\right)\left[\ell_{\left(1,2^{-1} r \zeta_{n}\right)}\right] \cdot\left[\zeta_{n}^{i}\right]\right)
\end{aligned}
$$


for any $(z, w) \in V(r) \backslash\left\{\left(1,2^{-1} r \zeta_{n}^{i}\right) \mid i \in \mathbb{N} \cap[0, n-1]\right\}$ and

$$
\begin{aligned}
F(z, w) & =\sum_{i=1}^{i_{(z, w)}} c_{0}\left(L_{\left(z, \zeta_{n}^{-i}\right) w}\right)=\sum_{i=1}^{i_{(z, w)}} c_{0}\left(\sum_{j=0}^{i_{(z, w)}-i-1}\left[\ell_{\left(1,2^{-1} r \zeta_{n}\right)}\right] \cdot\left[\zeta_{n}^{j}\right]\right) \\
& =\sum_{i=0}^{i_{(z, w)}-1} c_{0}\left(\sum_{j=0}^{i-1}\left[\ell_{\left(1,2^{-1} r \zeta_{n}\right)}\right] \cdot\left[\zeta_{n}^{j}\right]\right)=\sum_{i=0}^{i_{(z, w)}-1}\left(i_{(z, w)}-1-i\right) c_{0}\left(\ell_{\left(1,2^{-1} r \zeta_{n}\right)} \cdot\left[\zeta_{n}^{i}\right]\right)
\end{aligned}
$$

for any $(z, w) \in\left\{\left(1,2^{-1} r \zeta_{n}^{i}\right) \mid i \in \mathbb{N} \cap[0, n-1]\right\}$. In particular, we have $F\left(1,2^{-1} r\right)=0$ and $F\left(1,2^{-1} r \zeta_{n}\right)=0$.

We put $N:=\sum_{i=0}^{n-2}(n-i-1) c_{0}\left(\left[\ell_{\left(1,2^{-1} r \zeta_{n}\right)}\right] \cdot\left[\zeta_{n}^{i}\right]\right) \in \mathbb{Z}$. We define a $c_{1} \in \mathrm{C}_{\mathrm{m}}^{1}(V(r))$ by setting $c_{1}([\gamma]):=\left(d^{0} F\right)([\gamma])+N c_{\lambda}\left([\gamma]-L_{\mathscr{F}(\gamma)(0,1)}+L_{\mathscr{F}(\gamma)(1,0)}\right)$ for any

$$
\gamma \in \operatorname{Hom}_{\mathscr{C}}\left(\mathscr{A}_{V(r)}, \mathscr{A}_{\Delta^{1}}\right) \text {. }
$$

We verify $c_{1} \in \mathrm{Z}_{\mathrm{m}}^{1}(V(r))$ and $\left(\left[\zeta_{n}\right]-[1]\right) \cdot c_{1}=c_{0}$.

Let $\sigma \in \operatorname{Hom}_{\mathscr{C}}\left(\mathscr{A}_{V(r)}, \mathscr{A}_{\Delta^{2}}\right)$. We denote by $\gamma_{i} \in \operatorname{Hom}_{\mathscr{C}}\left(\mathscr{A}_{V(r)}, \mathscr{A}_{\Delta^{1}}\right)$ the composite of $\sigma$ and the morphism $\mathscr{A}_{\Delta^{2}} \rightarrow \mathscr{A}_{\Delta^{1}}$ in $\mathscr{C}$ associated with the $i$-th coface map $\Delta^{1} \hookrightarrow \Delta^{2}$ for an $i \in\{0,1\}$. We have $\partial[\sigma]=\sum_{i=0}^{2}(-1)^{i}\left[\gamma_{i}\right]$ and

$$
\begin{aligned}
\left(\mathscr{F}\left(\gamma_{0}\right)(0,1), \mathscr{F}\left(\gamma_{1}\right)(0,1), \mathscr{F}\left(\gamma_{2}\right)\right. & (0,1)) \\
= & \left(\mathscr{F}\left(\gamma_{2}\right)(1,0), \mathscr{F}\left(\gamma_{0}\right)(1,0), \mathscr{F}\left(\gamma_{1}\right)(1,0)\right) .
\end{aligned}
$$

Therefore we obtain

$$
\begin{aligned}
c_{1}(\partial[\sigma]) & =\left(d^{0} F\right)(\partial[\sigma])+N c_{\lambda}\left(\sum_{i=0}^{2}(-1)^{i}\left(\left[\gamma_{i}\right]-L_{\mathscr{F}\left(\gamma_{i}\right)(1,0)}+L_{\mathscr{F}\left(\gamma_{i}\right)(0,1)}\right)\right) \\
& =0+N c_{\lambda}(\partial[\sigma]+0)=0 .
\end{aligned}
$$

This implies $c_{1} \in A_{n, r}$. We compute $\left(\left[\zeta_{n}\right]-[1]\right) \cdot c_{1}$. Let $(z, w) \in V(r)$. We have

$$
\begin{aligned}
\left(\left(\left[\zeta_{n}\right]-[1]\right) \cdot d^{0} F\right)\left(L_{(z, w)}\right) & =\left(d^{0} F\right)\left(L_{(z, w)} \cdot\left[\zeta_{n}\right]-L_{(z, w)}\right) \\
& =F\left(\left[\left(z, \zeta_{n} w\right)\right]-\left[\left(1,2^{-1} r \zeta_{n}\right)\right]-[(z, w)]+\left[\left(1,2^{-1} r\right)\right]\right) \\
& =F\left(\left[\left(z, \zeta_{n} w\right)\right]-[(z, w)]\right) .
\end{aligned}
$$

If $i_{(z, w)} \neq n-1$, then we obtain $F\left(\left[\left(z, \zeta_{n} w\right)\right]-[(z, w)]\right)=c_{0}\left(L_{(z, w)}\right)$ by the definition of $F$. Suppose $i_{(z, w)}=n-1$. By $i_{z, \zeta_{n}^{i} w}=i-1 \neq n-1$ for any $i \in \mathbb{N} \cap[1, n-1]$, we have

$$
\begin{aligned}
F\left(\left[\left(z, \zeta_{n} w\right)\right]-[(z, w)]\right) & =-F\left(\left[\left(z, \zeta_{n}^{n} w\right)\right]-\left[\left(z, \zeta_{n} w\right)\right]\right) \\
& =-\sum_{i=1}^{n-1} F\left(\left[\left(z, \zeta_{n}^{i+1} w\right)\right]-\left[\left(z, \zeta_{n}^{i} w\right)\right]\right)=-\sum_{i=1}^{n-1} c_{0}\left(L_{\left(z, \zeta_{n}^{i} w\right)}\right)
\end{aligned}
$$


If $(z, w) \neq\left(1,2^{-1} r \zeta_{n}^{-1}\right)$, then we obtain

$$
\begin{aligned}
-\sum_{i=1}^{n-1} c_{0}\left(L_{\left(z, \zeta_{n}^{i} w\right)}\right)= & c_{0}\left(L_{(z, w)}\right)-\sum_{i=0}^{n-1} c_{0}\left(L_{\left(z, \zeta_{n}^{i} w\right)}\right) \\
= & c_{0}\left(L_{(z, w)}\right)-\sum_{i=0}^{n-1} c_{0}\left(\left[\ell_{\left(z, \zeta_{n} w\right)}\right] \cdot\left[\zeta_{n}^{i}\right]+\sum_{j=0}^{i-1}\left[\ell_{\left(1,2^{-1} r \zeta_{n}\right)}\right] \cdot\left[\zeta_{n}^{j}\right]\right) \\
= & c_{0}\left(L_{(z, w)}\right)-\left(\sum_{i=0}^{n-1}\left[\zeta_{n}^{i}\right] \cdot c_{0}\right)\left(\ell_{\left(z, \zeta_{n} w\right)}\right) \\
& \quad-\sum_{j=0}^{n-2}(n-1-j) c_{0}\left(\left[\ell_{\left(1,2^{-1} r \zeta_{n}\right)}\right] \cdot\left[\zeta_{n}^{j}\right]\right) \\
= & c_{0}\left(L_{(z, w)}\right)-0\left(\ell_{\left(z, \zeta_{n} w\right)}\right)-N=c_{0}\left(L_{(z, w)}\right)-N .
\end{aligned}
$$

On the other hand, if $(z, w)=\left(1,2^{-1} r \zeta_{n}^{-1}\right)$, then we obtain

$$
\begin{aligned}
-\sum_{i=1}^{n-1} c_{0}\left(L_{\left(z, \zeta_{n}^{i} w\right)}\right) & =-\sum_{i=0}^{n-2} c_{0}\left(L_{\left(1,2^{-1} r \zeta_{n}^{i}\right)}\right)=c_{0}\left(L_{\left(1,2^{-1} r \zeta_{n}^{-1}\right)}\right)-c_{0}\left(\sum_{i=0}^{n-1} L_{\left(1,2^{-1} r \zeta_{n}^{i}\right)}\right) \\
& =c_{0}\left(L_{(z, w)}\right)-c_{0}\left(\sum_{i=0}^{n-1} \sum_{j=0}^{i-1}\left[\ell_{\left(1,2^{-1} r \zeta_{n}\right)}\right] \cdot\left[\zeta_{n}^{j}\right]\right) \\
& =c_{0}\left(L_{(z, w)}\right)-c_{0}\left(\sum_{i=0}^{n-2}(n-1-i)\left[\ell_{\left(1,2^{-1} r \zeta_{n}\right)}\right] \cdot\left[\zeta_{n}^{i}\right]\right) \\
& =c_{0}\left(L_{(z, w)}\right)-N .
\end{aligned}
$$

It implies

$$
\left(\left(\left[\zeta_{n}\right]-[1]\right) \cdot d^{0} F\right)\left(L_{(z, w)}\right)= \begin{cases}c_{0}\left(L_{(z, w)}\right) & \text { if } i_{(z, w)} \neq n-1, \\ c_{0}\left(L_{(z, w)}\right)-N & \text { if } i_{(z, w)}=n-1 .\end{cases}
$$

Let $(z, w) \in V(r)$. We compute $c_{\lambda}\left(L_{(z, w)} \cdot\left[\zeta_{n}\right]-L_{\left(z, \zeta_{n} w\right)}\right)$. If $i_{(z, w)} \neq n-1$, then we have $L_{\left(z, \zeta_{n} w\right)}=L_{(z, w)} \cdot\left[\zeta_{n}\right]+\left[\ell_{\left(1,2^{-1} r \zeta_{n}\right)}\right]=0$ by the construction, and hence

$$
c_{\lambda}\left(L_{(z, w)} \cdot\left[\zeta_{n}\right]-L_{\left(z, \zeta_{n} w\right)}\right)=c_{\lambda}\left(\ell_{\left(1,2^{-1} r \zeta_{n}\right)}\right) .
$$

If $i_{(z, w)}=n-1$, then we have $L_{(z, w)} \cdot\left[\zeta_{n}\right]-L_{\left(z, \zeta_{n} w\right)}=\sum_{i=1}^{n-1}\left[\ell_{\left(1,2^{-1} r\right)}\right] \cdot\left[\zeta_{n}^{i}\right]$ and hence $c_{\lambda}\left(L_{(z, w)} \cdot\left[\zeta_{n}\right]-L_{\left(z, \zeta_{n} w\right)}\right)=c_{\lambda}\left(\left[\lambda_{r, 2^{-1} r}\right]-\left[\ell_{\left(1,2^{-1} r\right)}\right]\right)=1-c_{\gamma}\left(\ell_{\left(1,2^{-1} r\right)}\right)$ by Proposition 2.4 applied to $V(r)$ because

$$
\left(\sum_{i=0}^{n-1}\left[\mathscr{F}\left(\ell_{\left(1,2^{-1} r\right)}\right)\right] \cdot\left[\zeta_{n}^{i}\right]\right)-\left[\mathscr{F}\left(\lambda_{r, 2^{-1} r}\right)\right] \in \mathbb{Z}^{\oplus \mathrm{C}\left(\Delta^{1}, V(r)\right)}
$$

is a singular 1-boundary. We obtain

$$
c_{\lambda}\left(L_{(z, w)} \cdot\left[\zeta_{n}\right]-L_{\left(z, \zeta_{n} w\right)}\right)= \begin{cases}-\left[\ell_{\left(1,2^{-1} r \zeta_{n}\right)}\right] & \text { if } i_{(z, w)} \neq n-1, \\ 1-\left[\ell_{\left(1,2^{-1} r\right.} \zeta_{n}\right) & \text { if } i_{(z, w)}=n-1 .\end{cases}
$$


Let $\gamma \in \operatorname{Hom}_{\mathscr{C}}\left(\mathscr{A}_{V(r)}, \mathscr{A}_{\Delta^{1}}\right)$. By the argument above, we have

$$
\begin{aligned}
\left(\left(\left[\zeta_{n}\right]-[1]\right) \cdot d^{0} F\right)(\gamma) & \begin{array}{ll}
c_{0}\left(L_{\mathscr{F}(\gamma)(0,1)}-L_{\mathscr{F}(\gamma)(1,0)}\right) & \text { if } i_{\mathscr{F}(\gamma)(1,0)} \neq n-1 \neq i_{\mathscr{F}(\gamma)(0,1)}, \\
c_{0}\left(L_{\mathscr{F}(\gamma)(0,1)}-L_{\mathscr{F}(\gamma)(1,0)}\right)-N & \text { if } i_{\mathscr{F}(\gamma)(0,1)}=n-1 \neq i_{\mathscr{F}(\gamma)(1,0),} \\
c_{0}\left(L_{\mathscr{F}(\gamma)(0,1)}-L_{\mathscr{F}(\gamma)(1,0)}\right)+N & \text { if } i_{\mathscr{F}(\gamma)(0,1)} \neq n-1=i_{\mathscr{F}(\gamma)(1,0),} \\
c_{0}\left(L_{\mathscr{F}(\gamma)(0,1)}-L_{\mathscr{F}(\gamma)(1,0)}\right) & \text { if } i_{\mathscr{F}(\gamma)(1,0)}=n-1=i_{\mathscr{F}(\gamma)(0,1)},
\end{array} \\
= & \begin{cases}c_{0}([\gamma]) & \text { if } i_{\mathscr{F}(\gamma)(1,0)} \neq n-1 \neq i_{\mathscr{F}(\gamma)(0,1)}, \\
c_{0}([\gamma])-N & \text { if } i_{\mathscr{F}(\gamma)(0,1)}=n-1 \neq i_{\mathscr{F}(\gamma)(1,0),} \\
c_{0}([\gamma])+N & \text { if } i_{\mathscr{F}(\gamma)(0,1)} \neq n-1=i_{\mathscr{F}(\gamma)(1,0),} \\
c_{0}([\gamma]) & \text { if } i_{\mathscr{F}(\gamma)(1,0)}=n-1=i_{\mathscr{F}(\gamma)(0,1)},\end{cases}
\end{aligned}
$$

by $\partial\left(L_{(z, w)} \cdot\left[\zeta_{n}\right]-L_{\left(z, \zeta_{n} w\right)}+\left[\ell_{\left(1,2^{-1} r \zeta_{n}\right)}\right]\right)=0$. We obtain

$$
\begin{aligned}
& \left(\left(\left[\zeta_{n}\right]-[1]\right) \cdot c_{1}\right)(\gamma)-\left(\left(\left[\zeta_{n}\right]-[1]\right) \cdot d^{0} F\right)(\gamma) \\
& =N c_{\lambda}\left(\left([\gamma] \cdot\left[\zeta_{n}\right]-L_{\mathscr{F}(\gamma)(0,1) \cdot\left[\zeta_{n}\right]}+L_{\mathscr{F}(\gamma)(1,0) \cdot\left[\zeta_{n}\right]}\right)\right. \\
& \left.-\left([\gamma]-L_{\mathscr{F}(\gamma)(0,1)}+L_{\mathscr{F}(\gamma)(1,0)}\right)\right) \\
& =N c_{\lambda}\left(\left([\gamma]-L_{\mathscr{F}(\gamma)(0,1)}+L_{\mathscr{F}(\gamma)(1,0)}\right) \cdot\left[\zeta_{n}\right]\right) \\
& +N c_{\lambda}\left(L_{\mathscr{F}(\gamma)(0,1)} \cdot\left[\zeta_{n}\right]-L_{\mathscr{F}(\gamma)(0,1) \cdot\left[\zeta_{n}\right]}\right) \\
& -N c_{\lambda}\left(L_{\mathscr{F}(\gamma)(1,0)} \cdot\left[\zeta_{n}\right]-L_{\mathscr{F}(\gamma)(1,0) \cdot\left[\zeta_{n}\right]}\right) \\
& -N c_{\lambda}\left([\gamma]-L_{\mathscr{F}(\gamma)(0,1)}+L_{\mathscr{F}(\gamma)(1,0)}\right) \\
& =N c_{\lambda}\left(L_{\mathscr{F}(\gamma)(0,1)} \cdot\left[\zeta_{n}\right]-L_{\mathscr{F}(\gamma)(0,1) \cdot\left[\zeta_{n}\right]}\right) \\
& -N c_{\lambda}\left(L_{\mathscr{F}(\gamma)(1,0)} \cdot\left[\zeta_{n}\right]-L_{\mathscr{F}(\gamma)(1,0) \cdot\left[\zeta_{n}\right]}\right)
\end{aligned}
$$

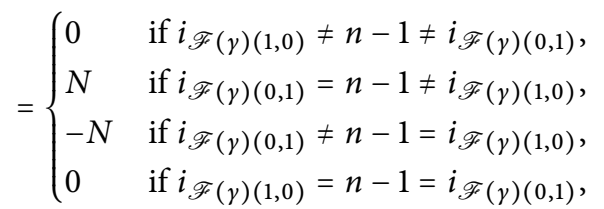

by Proposition 2.4 applied to $V(r)$, because the action of $\mu_{n}$ on $V(r)$ induces the trivial action on $\mathrm{H}_{1}(V(r))$. We obtain $\left(\left[\zeta_{n}\right]-[1]\right) \cdot c_{1}=c_{0}$. We define a map $\chi^{\prime}: \mu_{n} \rightarrow$ $A_{n, r}$ by setting $\chi^{\prime}\left(\zeta_{n}^{i}\right):=\sum_{j=0}^{i-1}\left[\zeta_{n}^{j}\right] \cdot c_{1}$ for any $i \in \mathbb{N} \cap[0, n-1]$. Then $\chi^{\prime}$ lies in $\mathrm{Z}^{1}\left(\mu_{n}, A_{n, r}\right)$, and satisfies $\left(\left[\zeta_{n}\right]-[1]\right) \cdot \chi^{\prime}=\chi$. We conclude that $\widehat{\mathrm{H}}^{1}\left(\mu_{n}, A_{n, r}\right)=\{0\}$.

Proof of Theorem 3.16. By definition, $d_{\widehat{\wp}}$ is a surjective continuous group homomorphism $G_{K, \widetilde{\wp}} \rightarrow \widehat{\mathbb{Z}}$, and $\bar{K}_{\wp}^{\times}$is a smooth $G_{K, \widetilde{\wp}}$-module. We identify $K_{\wp}^{\times}$with $\left(\bar{K}_{\widetilde{\wp}}^{\times}\right)^{G_{K, \widehat{\wp}}}$ by the group isomorphism in Corollary 3.11, and regard $v_{\wp}$ as a group homomorphism $\left(\bar{K}_{\widehat{\wp}}^{\times}\right)^{G_{K, \widehat{G}}} \rightarrow \widehat{\mathbb{Z}}$ whose image is $\mathbb{Z}$. 
First, we verify that $v_{\wp}$ is a Henselian valuation with respect to $d_{\widehat{\wp}}$. Let $H \subset G_{K, \widehat{\wp}}$ be an open subgroup. We put $f_{H}:=\#\left(\widehat{\mathbb{Z}} / d_{\widehat{\wp}}(H) \widehat{\mathbb{Z}}\right) \in \mathbb{N}_{>0}$, and denote by

$$
\operatorname{tr}_{H}:\left(\bar{K}_{\widehat{\wp}}^{\times}\right)^{H} \longrightarrow K_{\wp}^{\times}
$$

the trace map with respect to the action of cosets in $G_{K, \widehat{\wp}} / H$. It suffices to show that $v_{\wp}\left(\operatorname{im}\left(\operatorname{tr}_{H}\right)\right)=f_{H} \mathbb{Z}$.

Let $c \in\left(\bar{K}_{\widehat{\wp}}^{\times}\right)^{H}$. By Corollary 3.5, there is an $L \in M(K)$ such that for any

$$
\widetilde{L} \in M^{\mathrm{Gal}}(K)
$$

with $L \leq \widetilde{L}$, the image of $H$ by the canonical projection $G_{K, \widehat{\wp}} \rightarrow \operatorname{Gal}(\widetilde{L} / K)_{\wp_{\widetilde{L}}}$ coincides with $\operatorname{Gal}(\widetilde{L} / L)_{\wp_{\tilde{L}}}$. By Corollary 3.11 and the natural identification of

$$
\{\widetilde{L} \in M(K) \mid L \leq \widetilde{L}\}
$$

with $M(L), c$ is the image of a unique $c^{\prime} \in L_{\wp_{L}}^{\times}$. By the cofinality of $M^{\mathrm{Gal}}(K)$ in $M(K)$, there is an $\widetilde{L} \in M^{\mathrm{Gal}}(K)$ with $L \leq \widetilde{L}$. We have $f_{H} \mid f_{\wp \widetilde{L} / \wp}$ by the definition of $d_{\widehat{\wp}}$.

We take a tubular neighbourhood $j \in \operatorname{Hom}_{\mathscr{C}}\left(O_{K}, \mathscr{A}_{V(r)}\right)$ with $r \in(0,1]$ of $\wp$. Replacing $r$ by a smaller one, we may assume that $h_{\widetilde{L} / K}$ is unbranched at any point of $\operatorname{im}(\mathscr{F}(j)) \backslash \operatorname{im}(\wp)$. We put $\gamma:=\mu_{r, 2^{-1} r} \circ j \in \operatorname{Hom}_{\mathscr{C}}\left(O_{K}, \mathscr{A}_{\Delta^{1}}\right)$. We take a

$$
\tilde{\gamma} \in \operatorname{Hom}_{\mathscr{C}}\left(O_{\widetilde{L}}, \mathscr{A}_{\Delta^{1}}\right)
$$

with $\widetilde{\gamma} \circ l_{\widetilde{L} / K}=\gamma$ that actually exists by the covering homotopy property. We have

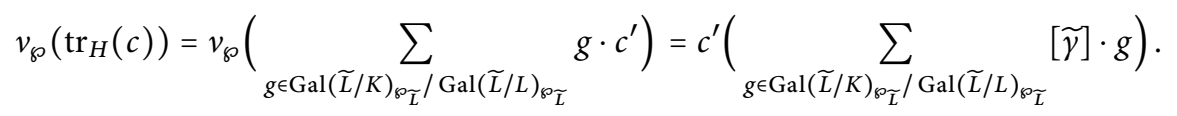

Replacing $r$ by a smaller one, we may assume that the pullback by $\mathscr{F}(j)$ of $h_{\widetilde{L} / K}$ restricted to a sufficiently small neighbourhood of $\operatorname{im}\left(\wp_{\widetilde{L}}\right)$ is given as the standard

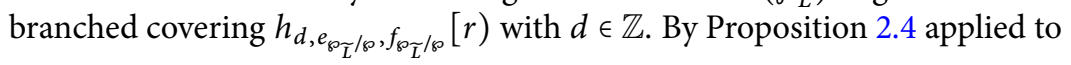

$$
V\left[r^{e_{\wp}^{-1} \widetilde{L}^{/ \wp}}\right)
$$

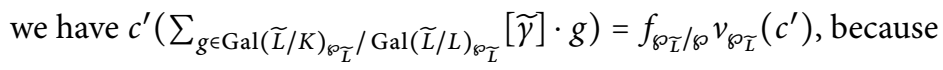

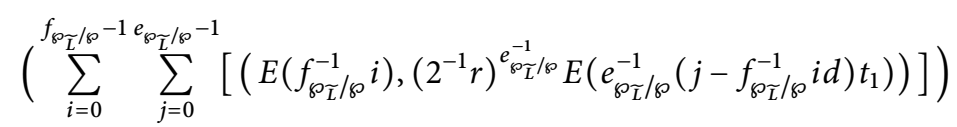

$$
\begin{aligned}
& -f_{\wp_{\widetilde{L}} / \wp}\left[\mathscr{F}\left(\mu_{r^{\prime}, 2^{-1} r^{\prime}}\right)\right] \in \mathbb{Z}^{\oplus \mathrm{C}\left(\Delta^{1}, V\left(r^{e^{-1} \widetilde{L} / \wp}\right)\right)}
\end{aligned}
$$

is a singular 1-boundary. We obtain $v_{\wp}\left(\operatorname{tr}_{H}(c)\right)=f_{\wp_{\widetilde{L}} / \wp} v_{\wp_{\widetilde{L}}}\left(c^{\prime}\right)$. It implies

$$
v_{\wp}\left(\operatorname{im}\left(\operatorname{tr}_{H}\right)\right) \subset f_{H} \mathbb{Z} \text {. }
$$

We show $f_{H} \in v_{\wp}\left(\operatorname{im}\left(\operatorname{tr}_{H}\right)\right)$. By the assumption, there is an $L \in M^{\mathrm{Gal}}(K)$ with $f_{H} \mid f_{\wp_{L} / \wp}$. We denote by $H \subset G_{K, \widehat{\wp}}$ the preimage of $f_{H} \mathbb{Z} / f_{\wp_{L} / \wp} \mathbb{Z} \subset \mathbb{Z} / f_{\wp_{L} / \wp} \mathbb{Z}$ by the composite of the canonical projection $G_{K, \widehat{\wp}} \rightarrow \operatorname{Gal}(L / K)_{\wp_{L}}$ and the surjective group homomorphism $\operatorname{red}_{\wp_{L} / \wp^{\circ}}: \operatorname{Gal}(L / K)_{\wp_{L}} \rightarrow \mathbb{Z} / f_{\wp_{L} / \wp} \mathbb{Z}(\operatorname{Section} 2.8)$. Then $H$ is an open normal subgroup of $G_{K, \widehat{\wp}}$ with $G_{K, \widehat{\wp}} / H \cong \mathbb{Z} / f_{H} \mathbb{Z}$. By Corollary 3.5 , there is an $L \in M^{\mathrm{Gal}}(K)$ such that for any $\widetilde{L} \in M^{\mathrm{Gal}}(K)$ with $L \leq \widetilde{L}$, the image of $H$ by 
the canonical projection $G_{K, \widetilde{\wp}} \rightarrow \operatorname{Gal}(\widetilde{L} / K)_{\wp_{\widetilde{L}}}$ coincides with $\operatorname{Gal}(\widetilde{L} / L)_{\wp_{\tau}}$. In particular, $H$ coincides with the kernel of the canonical projection $G_{K, \widehat{\wp}} \rightarrow \operatorname{Gal}(L / K)_{\wp_{L}}$ by $L \in M^{\mathrm{Gal}}(K)$. By the definition of $d_{\widehat{\varsigma}}$, we have $f_{\wp_{L} / \wp}=f_{H}$. By Theorem 3.14 (ii) applied to $L$ and $\wp_{L}$, there is a $c_{0} \in L_{\wp_{L}}^{\times}$with $v_{\wp_{L}}\left(c_{0}\right)=1$. We denote by $c_{1} \in \bar{K}_{\widehat{\wp}}^{\times}$the image of $c_{0}$. By the same computation above, we have $v_{\wp}\left(\operatorname{tr}_{H}\left(c_{1}\right)\right)=f_{\wp_{L} / \wp} v_{\wp_{L}}\left(c_{0}\right)=f_{H}$. Thus $v_{\wp}$ is a Henselian valuation with respect to $d_{\widehat{\wp}}$.

Next, we verify the class field axiom [Neu99, §IV (6.1)] for $\left(d_{\widehat{\wp}}, v_{\wp}\right)$. Let $H \subset G_{K, \widehat{\wp}}$ be an open normal subgroup such that $G_{K, \widetilde{\wp}} / H$ is isomorphic to $\mathbb{Z} / n \mathbb{Z}$ with $n \in \mathbb{N}_{>0}$. It suffices to verify $\widehat{\mathrm{H}}^{0}\left(G_{K, \widehat{\wp}} / H,\left(\bar{K}_{\widehat{\wp}}^{\times}\right)^{H}\right) \cong \mathbb{Z} / n \mathbb{Z}$ and $\widehat{\mathrm{H}}^{1}\left(G_{K, \widehat{\wp}} / H,\left(\bar{K}_{\widehat{\wp}}^{\times}\right)^{H}\right)=\{0\}$ [Neu99] §IV (3.7) Proposition. By Corollary 3.5, there is an $L \in M^{\mathrm{Gal}}(K)$ such that for any $\widetilde{L} \in M^{\mathrm{Gal}}(K)$ with $L \leq \widetilde{L}$, the image of $H$ by the canonical projection $G_{K, \widetilde{\varsigma}} \rightarrow$ $\operatorname{Gal}(\widetilde{L} / K)_{\wp_{\tilde{L}}}$ coincides with $\operatorname{Gal}(\widetilde{L} / L)_{\wp_{\tilde{L}}}$. In particular, $H$ coincides with the kernel of the canonical projection $G_{K, \widetilde{\wp}} \rightarrow \operatorname{Gal}(L / K)_{\wp_{L}}$ by $L \in M^{\text {Gal }}(K)$. By Corollary 3.11 and the natural identification of $\{\widetilde{L} \in M(K) \mid L \leq \widetilde{L}\}$ with $M(L)$, it suffices to verify $\widehat{\mathrm{H}}^{0}\left(\operatorname{Gal}(L / K)_{\wp_{L}}, L_{\wp_{L}}^{\times}\right) \cong \mathbb{Z} / n \mathbb{Z}$ and $\widehat{\mathrm{H}}^{1}\left(\mathrm{Gal}(L / K)_{\wp_{L}}, L_{\wp_{L}}^{\times}\right)=\{0\}$.

Replacing $r$ by a smaller one, we may assume that the pullback by $\mathscr{F}(j)$ of $h_{L / K}$ restricted to a sufficiently small neighbourhood of $\operatorname{im}\left(\wp_{L}\right)$ is given as the standard branched covering $h_{d, e_{\mathfrak{F}_{\tilde{L}} / \mathfrak{s}}, f_{\mathfrak{F}_{L} / \mathfrak{s}}}[r)$ with $d \in \mathbb{Z}$. By the elementary divisor theory, $\mathbb{Z}^{2}$ admits a $\mathbb{Z}$-linear basis $\left\{v_{0}, v_{1}\right\} \subset \mathbb{Z}^{2}$ such that $\mathbb{Z}\left(f_{\wp_{L} / 0}, 0\right)+\mathbb{Z}\left(d, e_{\wp_{\tilde{L}} / \wp}\right)$ is presented as $\mathbb{Z} c_{0} v_{0}+\mathbb{Z} c_{1} v_{1}$ for some $\left(c_{0}, c_{1}\right) \in \mathbb{N}_{>0}^{2}$ with $c_{0} \mid c_{1}$. In particular, we have

$$
\begin{aligned}
\mathbb{Z} / n \mathbb{Z} \cong \operatorname{Gal}(L / K)_{\wp_{L}} \cong \mathbb{Z}^{2} /\left(\mathbb{Z}\left(f_{\wp_{L} / \wp}, 0\right)+\mathbb{Z}\left(d, e_{\wp_{\tilde{I}} / \wp}\right)\right) \\
\\
=\left(\mathbb{Z} v_{0} \oplus \mathbb{Z} v_{1}\right) /\left(\mathbb{Z} c_{0} v_{0} \oplus \mathbb{Z} c_{1} v_{1}\right) \cong \mathbb{Z} / c_{0} \mathbb{Z} \oplus \mathbb{Z} / c_{1} \mathbb{Z},
\end{aligned}
$$

and hence $\left(c_{0}, c_{1}\right)=(1, n)$.

We put $\left(v_{0}, n v_{1}\right)=\left(a_{0,0}\left(f_{\wp_{L} / \wp}, 0\right)+a_{0,1}\left(d, e_{\wp_{\tilde{L}} / \wp}\right), a_{1,0}\left(f_{\wp_{L} / \wp}, 0\right)+a_{1,1}\left(d, e_{\wp_{\tilde{L}} / \wp}\right)\right)$ with $A:=\left(a_{i, j}\right)_{i, j=0}^{1} \in \mathrm{GL}_{2}(\mathbb{Z})$ and $\left(v_{0}, v_{1}\right)=\left(\left(b_{0,0}, b_{0,1}\right),\left(b_{1,0}, b_{1,1}\right)\right)$ with $B:=$ $\left(b_{i, j}\right)_{i, j=0}^{1} \in \mathrm{GL}_{2}(\mathbb{Z})$. By $A \in \mathrm{GL}_{2}(\mathbb{Z})$ and $B \in \mathrm{GL}_{2}(\mathbb{Z})$, the $\mathscr{C}$-morphisms

$$
\begin{aligned}
& h_{0}: V(r) \longrightarrow V(r), \\
& (z, w) \longmapsto\left(z^{\operatorname{det}(A)^{-1} a_{1,1}}\left(|w|^{-1} w\right)^{-\operatorname{det}(A)^{-1} a_{0,1}},|w| z^{-\operatorname{det}(A)^{-1} a_{1,0}}\left(|w|^{-1} w\right)^{\operatorname{det}(A)^{-1} a_{0,0}}\right)
\end{aligned}
$$

and

$$
\begin{aligned}
& h_{1}: V\left(r^{n^{-1}}\right) \longrightarrow V\left(r^{e_{\sigma_{\tilde{L}} / \mathfrak{s}}^{-1}}\right), \quad(z, w) \longmapsto \\
& \left(z^{\operatorname{det}(B)^{-1} b_{1,1}}\left(|w|^{-1} w\right)^{-\operatorname{det}(B)^{-1} b_{0,1}},|w|^{e^{-1} \tau_{\tilde{L}} / s} z^{-\operatorname{det}(B)^{-1} b_{1,0}}\left(|w|^{-1} w\right)^{\operatorname{det}(B)^{-1} b_{0,0}}\right)
\end{aligned}
$$

are diffeomorphisms with $h_{d, e_{\mathfrak{F}_{\tilde{L}} / \mathfrak{F}^{\circ}}, f_{\mathfrak{F}_{L} / \wp^{\circ}}}[r) \circ h_{0}=h_{1} \circ h_{0, c_{0}, c_{1}}[r)$. Therefore the assertion follows from Lemma 3.17.

Proof of Theorem 3.15 The second assertion is immediate from Theorem 3.16 and [Neu99, §IV (6.7) Theorem], as is the first assertion on the group isomorphism, because $K_{\wp}$ is isomorphic to $\mathbb{T}^{2}$ in $\widehat{\mathscr{C}}$ and the bijectivity of the given homomorphism is reduced to the covering of $\mathscr{A}_{V[r)}$ with $r \in(0,1]$ corresponding to $L / K$ and the reciprocity map for a $B \in M^{\mathrm{Gal}}\left(\mathscr{A}_{V[r)},\left\{p_{r}\right\}\right)$ associated with the class field theory for $\mathscr{A}_{V[r)}$ coincides with $\operatorname{nrs}_{B /\left(\mathscr{A}_{V[r},\left\{p_{r}\right\}\right),\left\{p_{r}\right\}}$ by the construction. The first assertion for 
the openness follows from the fact that the $\operatorname{nrs}_{L / K \wp_{\wp} L}$ factors through $\widehat{\mathrm{H}}_{\mathrm{m}}^{1}\left(K_{\wp}\right)$ by the definition, using $\mathrm{H}^{1}(V(r))$ with $r \in(0,1]$ and Proposition 2.4 applied to $V(r)$.

By Theorem 3.15 (i), we obtain the following.

Corollary 3.18 For any $\left(L, L^{\prime}\right) \in M^{\mathrm{Gal}}(K)^{2}$ with $L^{\prime} \leq L$ and $N_{L / K, \mathfrak{r}_{L}}=N_{L^{\prime} / K, \mathfrak{r}_{L^{\prime}}}$, $\iota_{L / L^{\prime}}$ induces an isomorphism $L_{\wp_{L^{\prime}}}^{\prime} \rightarrow L_{\wp_{L}}$ in $\widehat{\mathscr{C}}_{0}$.

Corollary 3.19 For any $L \in M^{\mathrm{Gal}}(K), \mathrm{Gal}(L / K)_{\wp_{L}}$ is Abelian.

By Corollary 3.19, we obtain the following.

Corollary 3.20 The group $G_{K, \widehat{\wp}}$ is Abelian.

\subsection{Global Class Field Theory}

We establish global class field theory in arithmetic topology in a way imitating the method in [NU]. Let $L$ be a Galois covering of $K$. By the definition of a covering of arithmetic manifolds, there is an $s \in \operatorname{Fin}\left(S_{K}\right)$ such that $h_{L / K}$ is unbranched outside $\operatorname{im}(s)$. In particular, we have $\operatorname{nrs}_{L / K, \wp}\left(\left(O_{K}\right)_{\wp}^{\times}\right)=\{1\}$ for any $\wp \in S_{K} \backslash s$. By $I_{K}=$ $\left(\prod_{\wp \in S_{K}} v_{\wp}\right)^{-1}\left(\mathbb{Z}^{\oplus S_{K}}\right)$ and $\operatorname{ker}\left(v_{K}\right)=U_{K}$, we have

$$
\left(\operatorname{nrs}_{L / K, \wp}\left(c_{\wp}\right)\right)_{\wp \in S_{K}} \in \underset{\wp \in S_{K}}{\oplus} \operatorname{Gal}(L / K)_{\wp}
$$

for any $\left(c_{\wp}\right)_{\wp \in S_{K}} \in I_{K}$.

Suppose that $L / K$ is Abelian so that the multiplication $\prod_{\wp \in S_{K}} g_{\wp} \in \mathrm{Gal}(L / K)$ makes sense for any $\left(g_{\wp}\right)_{\wp \in S_{K}} \in \bigoplus_{\wp \in S_{K}} \operatorname{Gal}(L / K)_{\wp}$. We consider the map

$$
\rho_{L / K}: I_{K} \longrightarrow \operatorname{Gal}(L / K), \quad\left(c_{\wp}\right)_{\wp \in S_{K}} \longmapsto \prod_{\wp \in S_{K}} \operatorname{nrs}_{L / K, \wp}\left(c_{\wp}\right) .
$$

By the construction, $\rho_{L / K}$ factors through the surjective group homomorphism

$$
\mathrm{H}_{1}(M \backslash \mathrm{im}(s)) \longrightarrow \operatorname{Gal}(L / K)
$$

in Proposition 3.2 (iii). Indeed, for a sufficiently small tubular neighbourhood

$$
j_{\wp} \in \operatorname{Hom}_{\mathscr{C}}\left(O_{K}, \mathscr{A}_{V\left[r_{\wp}\right)}\right)
$$

with $r_{\wp} \in(0,1]$ of each $\wp \in s$, the group homomorphism

$$
\begin{aligned}
\widetilde{\rho}_{s}: I_{K} \longrightarrow & \mathrm{H}_{1}(M \backslash \operatorname{im}(s)), \\
\left(c_{\wp}\right)_{\wp \in S_{K}} \longmapsto & \sum_{\wp \in S} c_{\wp}\left(\lambda_{r_{\wp}, 2^{-1} r_{\wp}} \circ j_{\wp}\right)\left[\mathscr{F}\left(\mu_{r_{\wp}, 2^{-1} r_{\wp}} \circ j_{\wp}\right)\right] \\
& -\sum_{\wp \in S_{K}} c_{\wp}\left(\mu_{r_{\wp}, 2^{-1} r_{\wp}} \circ j_{\wp}\right)\left[\mathscr{F}\left(\lambda_{r_{\wp}, 2^{-1} r_{\wp}} \circ j_{\wp}\right)\right]
\end{aligned}
$$

depends only on $s$, and the composite of $\widetilde{\rho}_{s}$ and the surjective group homomorphism $\mathrm{H}_{1}(M \backslash \operatorname{im}(s)) \rightarrow \operatorname{Gal}(L / K)$ coincide with $\rho_{L / K}$ by the definition of the local norm 
residue symbols. The compatible system $\left(\widetilde{\rho}_{s}\right)_{s \in \operatorname{Fin}\left(S_{K}\right)}$ yields a group homomorphism

$$
\begin{gathered}
\widetilde{\rho}: I_{K} \longrightarrow \lim _{s \in \operatorname{Fin}\left(S_{K}\right)} H_{1}(M \backslash \operatorname{im}(s)), \\
\left(c_{\wp}\right)_{\wp \in S_{K}} \longmapsto\left(\widetilde{\rho}_{s}\left(\left(c_{\wp}\right)_{\wp \in S_{K}}\right)\right)_{s \in \operatorname{Fin}\left(S_{K}\right) .}
\end{gathered}
$$

We show that $\widetilde{\rho}$ factors through $C_{K}$.

Proposition 3.21 If $K$ is orientable, then $\operatorname{ker}(\widetilde{\rho})$ contains $P_{K}+\prod_{\wp \in S_{K}} \widehat{\mathrm{B}}_{\mathrm{m}}^{1}\left(K_{\wp}\right)$.

In order to verify Proposition 3.21, it suffices to show the following.

Lemma 3.22 For any $s \in \operatorname{Fin}\left(S_{K}\right)$, if $O_{K}\left[s^{-1}\right]$ is orientable, then the equality

$$
\operatorname{ker}\left(\widetilde{\rho}_{s}\right)=P_{K}+\left(\prod_{\wp \in s} \widehat{\mathrm{B}}_{\mathrm{m}}^{1}\left(K_{\wp}\right) \times \prod_{\wp \in S_{K} \backslash s}\left(O_{K}\right)_{\wp}^{\times}\right)
$$

holds.

Proof By Theorem 3.14 and the definition of $\widetilde{\rho}_{s}$, we have

$$
\prod_{\wp \in s} \widehat{\mathrm{B}}_{\mathrm{m}}^{1}\left(K_{\wp}\right) \times \prod_{\wp \in S_{K} \backslash s}\left(O_{K}\right)_{\wp}^{\times} \subset \operatorname{ker}\left(\widetilde{\rho}_{s}\right),
$$

because the meridian of any $\wp \in S_{K} \backslash s$ is 0 in $\mathrm{H}_{1}(M \backslash \operatorname{im}(s))$.

First let $c \in K^{\times}$. We show $\widetilde{\rho}_{s}\left(l_{K}^{I}(c)\right)=0$. For this purpose, it suffices to consider the case where there is a $c_{0} \in \mathrm{Z}_{\mathrm{m}}^{1}\left(O_{K}\left[s^{-1}\right]\right)$ whose image in $K^{\times}$is $c$, because for any $s^{\prime} \in \operatorname{Fin}\left(S_{K}\right)$ with $s \subset s^{\prime}$, the image of $\widetilde{\rho}_{s^{\prime}}\left(\iota_{K}^{I}(c)\right)$ in $\mathrm{H}_{1}(M \backslash \operatorname{im}(s))$ coincides with $\tilde{\rho}_{s}\left(\iota_{K}^{I}(c)\right)$. For each $\wp \in s$, we take a tubular neighbourhood $j_{\wp} \in \operatorname{Hom}_{\mathscr{C}}\left(O_{K}, \mathscr{A}_{V\left[r_{\wp}\right)}\right)$ with $r_{\wp} \in(0,1]$ of $\wp$. Replacing $\left(r_{\wp}\right)_{\wp \in s}$ by a family of smaller ones, we can assume $\operatorname{im}\left(j_{\wp_{0}}\right) \cap \operatorname{im}\left(j_{\wp_{1}}\right)=\varnothing$ for any $\left(\wp_{0}, \wp_{1}\right) \in s^{2}$ with $\wp_{0} \neq \wp_{1}$. Furthermore, replacing $\left(r_{\wp}\right)_{\wp \in s}$ by $\left(2^{-1} r_{\wp}\right)_{\wp \in s}$, we can assume that $\left(j_{\wp}\right)_{\wp \in S}$ can be extended to a neighbourhood of the closures of the sources.

We put $X:=M \backslash \bigsqcup_{\wp \in s} \operatorname{im}\left(j_{\wp}\right)$. Then $X$ forms a three-dimensional $\mathscr{C}$-manifold such that $\partial X$ is homeomorphic to the disjoint union of copies of $S^{1} \times S^{1}$. We consider the diagram

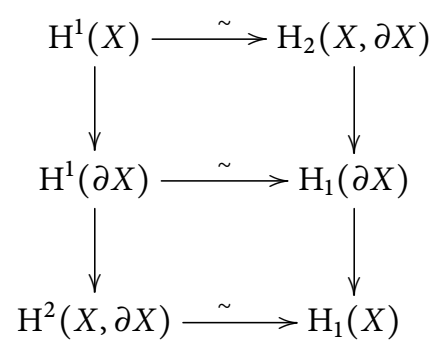

in $\mathrm{Ab}$ whose columns are given by the long exact sequences of relative cohomology and the relative homology and whose rows are given by the Poincaré-Lefschetz duality for fixed orientations. It is well known that this diagram commutes up to signature 
depending on the choice of orientations. The composite

$$
\begin{aligned}
& \mathrm{Z}_{\mathrm{m}}^{1}\left(O_{K}\left[s^{-1}\right]\right) \longrightarrow \mathrm{H}_{\mathrm{m}}^{1}\left(O_{K}\left[s^{-1}\right]\right) \\
& \stackrel{\sim}{\longrightarrow} \mathrm{H}^{1}(X) \longrightarrow \mathrm{H}^{1}(\partial X) \stackrel{\sim}{\longrightarrow} \mathrm{H}^{1}(M \backslash \operatorname{im}(s)) \\
& \mathrm{H}_{1}(\partial X) \longrightarrow \mathrm{H}_{1}(X) \stackrel{\sim}{\longrightarrow} \mathrm{H}_{1}(M \backslash \operatorname{im}(s))
\end{aligned}
$$

of the canonical projection $\mathrm{Z}_{\mathrm{m}}^{1}\left(O_{K}\left[s^{-1}\right]\right) \rightarrow \mathrm{H}_{\mathrm{m}}^{1}\left(O_{K}\left[s^{-1}\right]\right)$, the inverse of the isomorphism $\mathrm{H}^{1}(M \backslash \operatorname{im}(s)) \rightarrow \mathrm{H}_{\mathrm{m}}^{1}\left(O_{K}\left[s^{-1}\right]\right)$ in Proposition 2.4, the inverse of the isomorphism $\mathrm{H}^{1}(X) \rightarrow \mathrm{H}^{1}(M \backslash \operatorname{im}(s))$ associated with the inclusion $X \hookrightarrow M \backslash \operatorname{im}(s)$, the arrows $\mathrm{H}^{1}(X) \rightarrow \mathrm{H}^{1}(\partial X) \rightarrow \mathrm{H}_{1}(\partial X) \rightarrow \mathrm{H}_{1}(X)$, and the group isomorphism $\mathrm{H}_{1}(X) \rightarrow \mathrm{H}_{1}(M \backslash \operatorname{im}(s))$ associated with the inclusion $X \hookrightarrow M \backslash \operatorname{im}(s)$ coincides with the composite $\mathrm{Z}_{\mathrm{m}}^{1}\left(\mathrm{O}_{K}\left[s^{-1}\right]\right) \rightarrow K^{\times} \rightarrow \prod_{\wp \in s} K_{\wp}^{\times} \rightarrow \mathrm{H}_{1}(M \backslash \operatorname{im}(s))$ of the canonical homomorphism $Z_{\mathrm{m}}^{1}\left(O_{K}\left[s^{-1}\right]\right) \rightarrow K^{\times}, \prod_{\wp \in s} \iota_{\wp}^{\times}$, and $\widetilde{\rho}_{s}$ by definition. Since the composite of the arrows $\mathrm{H}^{1}(X) \rightarrow \mathrm{H}^{1}(\partial X) \rightarrow \mathrm{H}_{1}(\partial X) \rightarrow \mathrm{H}_{1}(X)$ in the diagram coincides with the composite

$$
\mathrm{H}^{1}(X) \longrightarrow \mathrm{H}^{1}(\partial X) \longrightarrow \mathrm{H}_{2}(X, \partial X) \longrightarrow \mathrm{H}_{1}(X)
$$

of the arrows in the diagram up to signature, it is the zero homomorphism. We obtain $\widetilde{\rho}_{s}\left(\iota_{K}^{I}(c)\right)=0\left(c_{0}\right)=0$. This implies $P_{K}+\left(\prod_{\wp \in s} \widehat{\mathrm{B}}_{\mathrm{m}}^{1}\left(K_{\wp}\right) \times \prod_{\wp \in S_{K} \backslash s}\left(O_{K}\right)_{\wp}^{\times}\right) \subset \operatorname{ker}\left(\widetilde{\rho}_{s}\right)$.

Next, let $\left(c_{\wp}\right)_{\wp \in S_{K}} \in \operatorname{ker}\left(\widetilde{\rho}_{s}\right)$. We will show

$$
\left(c_{\wp}\right)_{\wp \in S_{K}} \in P_{K}+\left(\prod_{\wp \in s} \widehat{\mathrm{B}}_{\mathrm{m}}^{1}\left(K_{\wp}\right) \times \prod_{\wp \in S_{K} \backslash s}\left(O_{K}\right)_{\wp}^{\times}\right) .
$$

We take an $s^{\prime} \in \operatorname{Fin}\left(S_{K}\right)$ with $s \subset s^{\prime}$ and $c_{\wp} \in\left(O_{K}\right)_{\wp}^{\times}$for any $\wp \in S_{K} \backslash s^{\prime}$. We denote by $X^{\prime} \subset M$ the complement of sufficiently small tubular neighbourhoods of $s^{\prime}$, by $X \subset M$ the complement of the tubular neighbourhoods of $s$, by $\bar{V} \subset M$ the closure of $X \backslash X^{\prime}$, and by $U^{\prime} \subset I_{K}$ the preimage of $\left(\prod_{\wp \in s^{\prime}} K_{\wp}^{\times}\right) \times\left(\prod_{\wp \in S_{K} \backslash s^{\prime}}\left(O_{K}\right)_{\wp}^{\times}\right) \subset \prod_{\wp \in S_{K}} K_{\wp}^{\times}$. We consider the same diagram above corresponding to $X^{\prime}$. The composite

$$
\begin{aligned}
U^{\prime} \hookrightarrow \longrightarrow \prod_{\wp \in S_{K}} K_{\wp}^{\times} \longrightarrow \prod_{\wp \in S_{K}} \widehat{\mathrm{H}}_{\mathrm{m}}^{1}\left(K_{\wp}\right) \stackrel{\sim}{\longrightarrow} \prod_{\wp \in S_{K}} \mathbb{Z}^{2} \longrightarrow \prod_{\wp \in s} \mathbb{Z}^{2} \stackrel{\sim}{\longrightarrow} \mathrm{H}_{1}\left(\partial X^{\prime}\right) \\
\longrightarrow \mathrm{H}_{1}\left(X^{\prime}\right) \stackrel{\sim}{\longrightarrow} \mathrm{H}_{1}\left(M \backslash \operatorname{im}\left(s^{\prime}\right)\right)
\end{aligned}
$$

of the direct product of the canonical projections $K_{\wp}^{\times} \rightarrow \widehat{\mathrm{H}}_{\mathrm{m}}^{1}\left(K_{\wp}\right)$ with $\wp \in S_{K}$ restricted to $U^{\prime}$, the direct product of the group isomorphisms $\widehat{\mathrm{H}}_{\mathrm{m}}^{1}\left(K_{\wp}\right) \rightarrow \mathbb{Z}^{2}$ with $\wp \in S_{K}$ in Theorem 3.14 (ii), the canonical projection $\prod_{\wp \in S_{K}} \mathbb{Z}^{2} \rightarrow \prod_{\wp \in \mathcal{s}^{\prime}} \mathbb{Z}^{2}$, the group isomorphism $\prod_{\wp \in s^{\prime}} \mathbb{Z}^{2} \rightarrow \mathrm{H}_{1}\left(\partial X^{\prime}\right)$ sending the canonical $\mathbb{Z}$-linear basis to the $\mathbb{Z}$-linear basis given by the meridians and the longitude multiplied by -1 , the arrow $\mathrm{H}_{1}\left(\partial X^{\prime}\right) \rightarrow \mathrm{H}_{1}\left(X^{\prime}\right)$ in the diagram, and the group isomorphism

$$
\mathrm{H}_{1}\left(X^{\prime}\right) \rightarrow \mathrm{H}_{1}\left(M \backslash \operatorname{im}\left(s^{\prime}\right)\right)
$$

associated with the inclusion $X^{\prime} \hookrightarrow M \backslash \mathrm{im}\left(s^{\prime}\right)$ coincides with $\widetilde{\rho}_{s^{\prime}}$ restricted to $U^{\prime}$ by definition. By $\widetilde{\rho}_{s}\left(\left(c_{\wp}\right)_{\wp \in S_{K}}\right)=0$ and a standard argument with the Mayer-Vietoris exact sequence, there is a $\mu \in \mathrm{H}_{1}\left(\partial X^{\prime} \backslash \partial X\right)$ whose image in $\mathrm{H}_{1}\left(X^{\prime}\right)$ is $\widetilde{\rho}_{s^{\prime}}\left(\left(c_{\wp}\right)_{\wp \in S_{K}}\right)$ and whose image in $\mathrm{H}_{1}(\bar{V})$ is 0 . By Theorem 3.14 (ii), there is a $\left(c_{\wp}^{\prime}\right)_{\wp \in S_{K}} \in I_{K}$ with $c_{\wp}^{\prime}=0$ for any $\wp \in S_{K} \backslash\left(s^{\prime} \backslash s\right)$ whose image by $\rho_{s^{\prime}}$ coincides with the image of $\mu$, i.e., $\widetilde{\rho}_{s^{\prime}}\left(\left(c_{\wp}\right)_{\wp \in S_{K}}\right)$. Since the left row of the diagram is exact, there is a $c^{\prime \prime} \in K^{\times}$ 
whose image in $\mathrm{H}^{1}\left(\partial X^{\prime}\right)$ coincides with that of $\left(c_{\wp}\right)_{\wp \in S_{K}}-\left(c_{\wp}^{\prime}\right)_{\wp \in S_{K}} \in \operatorname{ker}\left(\widetilde{\rho}_{s^{\prime}}\right)$ again by Theorem 3.14 (ii). We obtain

$$
\left(c_{\wp}\right)_{\wp \in S_{K}} \in \iota_{K}^{I}(c)+\left(c_{\wp}^{\prime}\right)_{\wp \in S_{K}}+\prod_{\wp \in S_{K}} \widehat{\mathrm{B}}_{\mathrm{m}}^{1}\left(K_{\wp}\right) \subset P_{K}+\left(\prod_{\wp \in s} \widehat{\mathrm{B}}_{\mathrm{m}}^{1}\left(K_{\wp}\right) \times \prod_{\wp \in S_{K} \backslash s}\left(O_{K}\right)_{\wp}^{\times}\right) .
$$

This implies $\operatorname{ker}\left(\widetilde{\rho}_{s}\right)=P_{K}+\left(\prod_{\wp \in s} \widehat{\mathrm{B}}_{\mathrm{m}}^{1}\left(K_{\wp}\right) \times \prod_{\wp \in S_{K} \backslash s}\left(O_{K}\right)_{\wp}^{\times}\right)$.

Suppose in the following that $K$ is orientable. Then by Proposition 3.21, $\rho_{L / K}$ induces a group homomorphism $\mathrm{nrs}_{L / K}: C_{K} \rightarrow \operatorname{Gal}(L / K)$ that is analogous to the norm residue symbol in global class field theory in number theory. By Theorem 3.12, the trace map $I_{L} \rightarrow I_{L}^{\mathrm{Gal}(L / K)}, c \mapsto \sum_{g \in \operatorname{Gal}(L / K)} g \cdot c$ induces a group isomorphism $\operatorname{tr}_{L / K}: I_{L} \rightarrow I_{K}$. We denote by $N_{L / K} \subset C_{K}$ the image of $\operatorname{im}\left(\operatorname{tr}_{L / K}\right) \subset I_{K}$.

We denote by $M^{\mathrm{Ab}}(K) \subset M(K)$ the subset of Abelian coverings of $K$. We verify global class field theory in arithmetic topology.

Theorem 3.23 (Global class field theory) If $K$ is an orientable closed stably admissible arithmetic manifold, then the following assertions hold.

(i) For any $L \in M^{\mathrm{Ab}}(K), N_{L / K}$ is an open subgroup of $C_{K}$ of finite index, and $\mathrm{nrs}_{L / K}$ induces a group isomorphism $C_{K} / N_{L / K} \rightarrow \operatorname{Gal}(L / K)$.

(ii) For any open subgroup $N \subset C_{K}$ of finite index, there is a unique $L \in M^{\mathrm{Ab}}(K)$ with $N=N_{L / K}$.

Proof First let $L \in M^{\mathrm{Ab}}(K)$. By the assumption of stable admissibility, there is an $s \in \operatorname{Fin}\left(S_{K}\right)$ such that $\left\{\operatorname{Frob}_{\wp} \mid \wp \in s\right\}$ and $\left\{\operatorname{Frob}_{\mathscr{P}} \mid \mathscr{P} \in h_{L / K}^{*}(s)\right\}$ generate $\mathrm{H}_{1}(M)$ and $\mathrm{H}_{1}\left(\mathscr{F}_{0}\left(O_{L}\right)\right)$, respectively, because the closedness of $K$ ensures that $\mathrm{H}_{1}(M)$ and $\mathrm{H}_{1}\left(\mathscr{F}_{0}\left(O_{L}\right)\right)$ are finitely generated Abelian groups. Replacing $s$ by a larger one, we can assume that $h_{L / K}$ is unbranched outside $\operatorname{im}(s)$. We show that $\operatorname{nrs}_{L / K}$ factors through $C_{K} / N_{L / K}$. For this purpose, it suffices to verify that the diagram

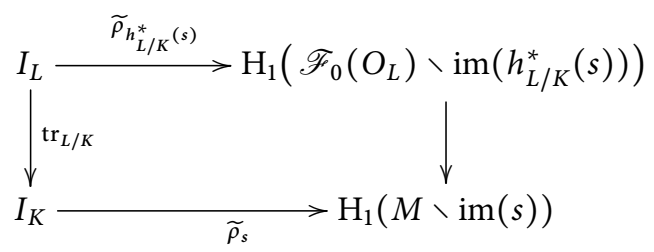

commutes, where the right vertical arrow is the group homomorphism $\tau$ associated with the restriction of $h_{L / K}$, because the horizontal arrows are surjective by the choice of $s$.

Let $\left(\widetilde{c} \mathscr{P}_{\mathscr{P} \in S_{L}} \in I_{L}\right.$. For each $\wp \in S_{K}$, we take a tubular neighbourhood

$$
j_{\wp} \in \operatorname{Hom}_{\mathscr{C}}\left(O_{K}, \mathscr{A}_{V\left[r_{\wp}\right)}\right)
$$

with $r_{\wp} \in(0,1]$ of $\wp$. For each $\mathscr{P} \in S_{L}$, we take a tubular neighbourhood

$$
j_{\mathscr{P}} \in \operatorname{Hom}_{\mathscr{C}}\left(O_{L}, \mathscr{A}_{V\left[r_{\mathscr{P}}\right)}\right)
$$

with $r_{\mathscr{P}} \in(0,1]$ of $\mathscr{P}$, and abbreviate $\iota_{L / K}^{*} \mathscr{P}$ to $\mathscr{P}_{K}$. Replacing $\left(r_{\mathscr{P}}\right)_{\mathscr{P} \in S_{L}}$ by a family of smaller ones, we can assume that for each $\mathscr{P} \in S_{L}$, there is a $\widetilde{\mathcal{c}} \mathscr{P}, 0 \in \mathrm{Z}_{\mathrm{m}}^{1}\left(\left.O_{L}\right|_{\mathrm{im}\left(j_{\mathscr{P}}\right)}\right)$ 
whose image in $L_{\mathscr{P}}^{\times}$is $\widetilde{\mathcal{c}} \mathscr{P}$. Furthermore, replacing $\left(r_{\wp}\right)_{\wp \in S_{K}}$ and $\left(r_{\mathscr{P}}\right)_{\mathscr{P} \in S_{L}}$ by families of smaller ones, we can assume $r_{\mathscr{P}}=r_{\mathscr{P} / \mathscr{\wp}}^{e^{-1}}$ for any $\mathscr{P} \in S_{L}, \operatorname{im}\left(j_{\wp}\right) \cap \operatorname{im}(s)=$ $\operatorname{im}(\wp)$ for any $\wp \in s$, and $\operatorname{im}\left(j_{\wp}\right) \cap \operatorname{im}(s)=\varnothing$ for any $\wp \in S_{L} \backslash s$. Then for any $\mathscr{P} \in S_{L}$,

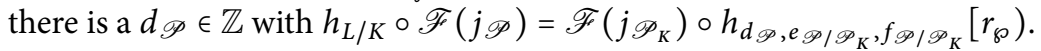

In order to show $\widetilde{\rho}_{s}\left(\operatorname{tr}_{L / K}\left(\left(\widetilde{c}_{\mathscr{P}}\right) \mathscr{P}_{\in} \in S_{L}\right)\right)=\tau\left(\widetilde{\rho}_{h_{L / K}^{*}(s)}\left(\left(\widetilde{c}_{\mathscr{P}}\right)_{\mathscr{P} \in S_{L}}\right)\right)$, we prepare the notation. Let $\wp \in S_{K}$. We denote by $j_{\wp}^{\circ} \in \operatorname{Hom}_{\mathscr{C}}\left(\left.O_{K}\right|_{\operatorname{im}\left(j_{\wp}\right) \backslash \operatorname{im}(\wp)}, \mathscr{A}_{V\left(r_{\wp}\right)}\right)$ the morphism given by the restriction of $\mathscr{F}\left(j_{\wp}\right)$. We put

$$
\begin{aligned}
& \mu_{\wp}:=\mu_{r_{\wp}, 2^{-1} r_{\wp}} \circ j_{\wp}^{\circ} \in \operatorname{Hom}_{\mathscr{C}}\left(\left.O_{K}\right|_{\operatorname{im}\left(j_{\wp}\right) \backslash \operatorname{im}(\wp)}, \mathscr{A}_{\Delta^{1}}\right), \\
& \lambda_{\wp}:=\lambda_{r_{\wp}, 2^{-1} r_{\wp}} \circ j_{\wp}^{\circ} \in \operatorname{Hom}_{\mathscr{C}}\left(\left.O_{K}\right|_{\operatorname{im}\left(j_{\wp}\right) \backslash \operatorname{im}(\wp)}, \mathscr{A}_{\Delta^{1}}\right) .
\end{aligned}
$$

Let $\mathscr{P} \in S_{L}$. We denote by $j_{\mathscr{P}}^{\circ} \in \operatorname{Hom}_{\mathscr{C}}\left(\left.O_{L}\right|_{\operatorname{im}\left(j_{\mathscr{P}}\right) \backslash \operatorname{im}(\mathscr{P})}, \mathscr{A}_{V\left(r_{\mathscr{P}}\right)}\right)$ the morphism given by the restriction of $\mathscr{F}(j \mathscr{P})$, and by

$$
\tau_{\mathscr{P}}: \mathrm{H}_{1}\left(\operatorname{im}\left(j_{\mathscr{P}} \backslash \mathrm{im}(\mathscr{P})\right) \longrightarrow \mathrm{H}_{1}\left(\operatorname{im}\left(j_{\mathscr{P}_{K}}\right) \backslash \operatorname{im}\left(\mathscr{P}_{K}\right)\right)\right.
$$

the group homomorphism associated with the restriction of $h_{L / K}$. We put

$$
\begin{aligned}
\mu_{\mathscr{P}} & :=\mu_{r_{\mathscr{P}}, 2^{-1} r_{\mathscr{P}}} \circ j_{\mathscr{P}}^{\circ} \in \operatorname{Hom}_{\mathscr{C}}\left(\left.O_{L}\right|_{\mathrm{im}\left(j_{\mathscr{P}}\right) \backslash \mathrm{im}(\mathscr{P})}, \mathscr{A}_{\Delta^{1}}\right), \\
\lambda_{\mathscr{P}} & \left.:=\lambda_{r_{\mathscr{P}}, 2^{-1} r_{\mathscr{P}}} \circ j_{\mathscr{P}}^{\circ} \in \operatorname{Hom}_{\mathscr{C}}\left(\left.O_{L}\right|_{\mathrm{im}\left(j_{\mathscr{P}}\right) \backslash \mathrm{im}(\mathscr{P})}, \mathscr{A}_{\Delta^{1}}\right)\right) .
\end{aligned}
$$

Let $i \in \mathbb{N}$. We denote by $m_{\mathscr{P}, i} \in \operatorname{Hom}_{\mathscr{C}}\left(\mathscr{A}_{V\left(r_{\mathscr{P}}\right)}, \mathscr{A}_{\Delta^{1}}\right)$ the morphism given by the $\mathscr{C}$-morphism

$$
\Delta^{1} \longrightarrow V\left(r_{\mathscr{P}}\right), \quad\left(t_{0}, t_{1}\right) \longmapsto\left(\zeta_{f_{\mathscr{P} / \mathscr{P}_{K}}^{i}}^{i} 2^{-1} r_{\mathscr{P}} E\left(e_{\mathscr{P} / \mathscr{P}_{K}}^{-1} i t_{1}\right)\right),
$$

and by $\ell_{\mathscr{P}, i} \in \operatorname{Hom}_{\mathscr{C}}\left(\mathscr{A}_{V\left(r_{\mathscr{P}}\right)}, \mathscr{A}_{\Delta^{1}}\right)$ the morphism given by the $\mathscr{C}$-morphism

$$
\Delta^{1} \longrightarrow V\left(r_{\mathscr{P}}\right), \quad\left(t_{0}, t_{1}\right) \longmapsto\left(E\left(f_{\mathscr{P} / \mathscr{P}_{K}}^{-1} i t_{1}\right), 2^{-1} r_{\mathscr{P}} E\left(-e_{\mathscr{P} / \mathscr{P}_{K}}^{-1} f_{\mathscr{P} / \mathscr{P}_{K}}^{-1} d_{\mathscr{P}} i t_{1}\right)\right)
$$

We put

$$
\begin{aligned}
& \mu_{\mathscr{P}, i}:=m_{\mathscr{P}, i} \circ j_{\mathscr{P}} \in \operatorname{Hom}_{\mathscr{C}}\left(\left.O_{L}\right|_{\operatorname{im}(j \mathscr{P}) \backslash \operatorname{im}(\wp)}, \mathscr{A}_{\Delta^{1}}\right), \\
& \lambda_{\mathscr{P}, i}:=\ell_{\mathscr{P}, i} \circ j_{\mathscr{P}} \in \operatorname{Hom}_{\mathscr{C}}\left(\left.O_{L}\right|_{\operatorname{im}\left(j_{\mathscr{P}}\right) \backslash \operatorname{im}(\wp)}, \mathscr{A}_{\Delta^{1}}\right) .
\end{aligned}
$$

Then we have

$$
\begin{aligned}
& \tau_{\mathscr{P}}\left(\left[\mathscr{F}\left(\mu_{\mathscr{P}}\right)\right]\right)=e_{\mathscr{P}} \mid \mathscr{P}_{K}\left[\mathscr{F}\left(\mu_{\mathscr{P}_{K}}\right)\right], \\
& \tau_{\mathscr{P}}\left(\left[\mathscr{F}\left(\lambda_{\mathscr{P}}\right)\right]\right)=f_{\mathscr{P} / \mathscr{P}_{K}}\left[\mathscr{F}\left(\lambda_{\mathscr{P}_{K}}\right)\right]+d_{\mathscr{P}}\left[\mathscr{F}\left(\mu_{\mathscr{P}_{K}}\right)\right],
\end{aligned}
$$

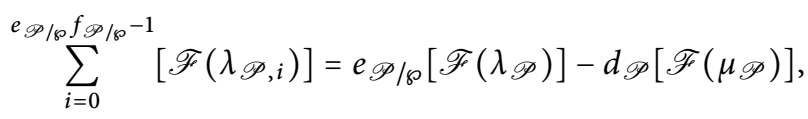

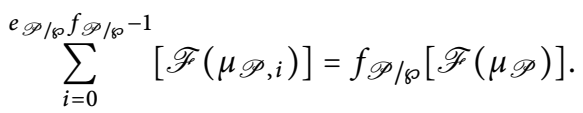


Therefore by Proposition 2.4, we obtain

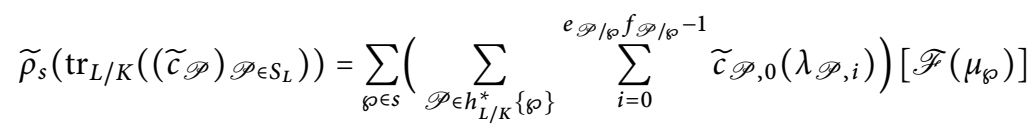

$$
\begin{aligned}
& -\sum_{\wp \in S_{K}}\left(\sum_{\mathscr{P} \in h_{L / K}^{*}\{\wp\}} \sum_{i=0}^{e_{\mathscr{P} / \wp} f_{\mathscr{P} / \wp}-1} \widetilde{\mathcal{C}} \mathscr{P}, 0(\mu \mathscr{P}, i)\right)\left[\mathscr{F}\left(\lambda_{\wp}\right)\right] \\
& =\sum_{\mathscr{P} \in h_{L / K}^{*}(s)}\left(v_{\mathscr{P}}^{\log }\left(\widetilde{c}_{\mathscr{P}}\right) e_{\mathscr{P}} / \mathscr{P}_{K}-v_{\mathscr{P}}\left(\widetilde{c}_{\mathscr{P}}\right) d_{\mathscr{P}}\right)\left[\mathscr{F}\left(\mu_{\mathscr{P}_{K}}\right)\right] \\
& -\sum_{\mathscr{P} \in S_{L}} v_{\mathscr{P}}\left(\widetilde{c}_{\mathscr{P}}\right) f_{\mathscr{P} / \mathscr{P}_{K}}\left[\mathscr{F}\left(\lambda_{\mathscr{P}_{K}}\right)\right]
\end{aligned}
$$

and

$$
\begin{aligned}
& \tau\left(\widetilde{\rho}_{h_{L / K}^{*}(s)}\left((\widetilde{\mathcal{c}} \mathscr{P}) \mathscr{P} \in S_{L}\right)\right)=\sum_{\mathscr{P} \in h_{L / K}^{*}(s)} v_{\mathscr{P}}^{\log }(\widetilde{\mathcal{c}} \mathscr{P}) \tau_{\mathscr{P}}([\mathscr{F}(\mu \mathscr{P})]) \\
& -\sum_{\mathscr{P} \in S_{L}} v_{\mathscr{P}}\left(\widetilde{\mathcal{C}}_{\mathscr{P}}\right) \tau_{\mathscr{P}}\left(\left[\mathscr{F}\left(\lambda_{\mathscr{P}}\right)\right]\right) \\
& =\sum_{\mathscr{P} \in h_{L / K}^{*}(s)} v_{\mathscr{P}}^{\log }(\widetilde{c} \mathscr{P}) e_{\mathscr{P}} / \mathscr{P}_{K}\left[\mathscr{F}\left(\mu \mathscr{P}_{K}\right)\right] \\
& -\sum_{\mathscr{P} \in S_{L}} v_{\mathscr{P}}\left(\widetilde{c}_{\mathscr{P}}\right)\left(f_{\mathscr{P} / \mathscr{P}_{K}}\left[\mathscr{F}\left(\lambda_{\mathscr{P}_{K}}\right)\right]+d \mathscr{P}\left[\mathscr{F}\left(\mu \mathscr{P}_{K}\right)\right]\right) .
\end{aligned}
$$

This implies

$$
\begin{aligned}
\tilde{\rho}_{s}\left(\operatorname{tr}_{L / K}\left((\widetilde{c} \mathscr{P}) \mathscr{P} \in S_{L}\right)\right)= & \tau\left(\widetilde{\rho}_{h_{L / K}^{*}(s)}\left(\left(\widetilde{c}_{\mathscr{P}}\right) \mathscr{P} \in S_{L}\right)\right) \\
& -\sum_{\mathscr{P} \in S_{L} \backslash h_{L / K}^{*}(s)} d \mathscr{P} v \mathscr{P}(\widetilde{c} \mathscr{P})\left[\mathscr{F}\left(\mu \mathscr{P}_{K}\right)\right] \\
= & \tau\left(\widetilde{\rho}_{h_{L / K}^{*}(s)}\left(\left(\widetilde{c}_{\mathscr{P}}\right)_{\mathscr{P} \in S_{L}}\right)\right)
\end{aligned}
$$

because $\left[\mathscr{F}\left(\mu_{\wp}\right)\right]=0 \in \mathrm{H}_{1}(M \backslash \operatorname{im}(s))$ for any $\wp \in S_{K} \backslash s$. Thus the diagram commutes. The openness of $N_{L / K}$ follows from Theorem 3.21.

Next let $N \subset C_{K}$ be an open subgroup of finite index. By the definition of the topology of $C_{K}$, there is an $(s, n) \in \operatorname{Fin}\left(S_{K}\right) \times \mathbb{N}_{>0}$ such that the preimage of $N$ in $I_{K}$ contains $\left(\prod_{\wp \in s}\left(\left(O_{K}\right)_{\wp}^{\times} \cap\left(v_{\wp}^{\log }\right)^{-1}(n \mathbb{Z})\right) \times\left(\prod_{\wp \in S_{K} \backslash s}\left(O_{K}\right)_{\wp}^{\times}\right)\right.$. In particular, the preimage of $N$ in $I_{K}$ contains $\operatorname{ker}\left(\widetilde{\rho}_{s}\right)$ by Lemma 3.22. By assertion (i), $\operatorname{im}\left(\widetilde{\rho}_{s}\right)$ is dense in $\mathrm{H}_{1}(M \backslash \mathrm{im}(s))$ with respect to the topology generated by the set of subsets of the form $c+U$ for a $c \in \mathrm{H}_{1}(M \backslash \operatorname{im}(s))$ and a subgroup $U \subset \mathrm{H}_{1}(M \backslash \operatorname{im}(s))$ of finite index. Since $\mathrm{H}_{1}(M \backslash \operatorname{im}(s))$ is a finitely generated Abelian group, every subgroup of it is closed, and hence $\widetilde{\rho}_{s}$ is surjective. Therefore, there is an $L \in M^{\mathrm{Ab}}(K)$ such that $\mathscr{F}_{0}\left(\iota_{L / K}\right)$ is unbranched outside $\operatorname{im}(s)$ and the preimage by $\widetilde{\rho}_{s}$ of the kernel of the surjective group homomorphism $\mathrm{H}_{1}(M \backslash \operatorname{im}(s)) \rightarrow \operatorname{Gal}(L / K)$ coincides with $N$. This implies $N_{L / K}=\operatorname{ker}\left(\operatorname{nrs}_{L / K}\right)=N$. The uniqueness of such an $L$ follows from assertion (i) because $M^{\mathrm{Ab}}(K)$ is directed with respect to the order $\leq$ on $M(K)$. 


\subsection{Ideal-Theoretic Class Field Theory}

We formulate ideal-theoretic class field theory for arithmetic topology in the sense of [Neu99, §VI 7]. To begin with, since $\left\{I_{K}^{m} \mid m \in \mathbb{N}_{>0}^{\oplus S_{K}}\right\}$ forms a fundamental system of neighbourhoods of $0 \in I_{K}$, we have the following.

\section{Proposition 3.24}

(i) For any $m \in \mathbb{N}_{>0}^{\oplus S_{K}}, C_{K}^{m} \subset C_{K}$ is an open subgroup.

(ii) For any open subgroup $N \subset C_{K}$, there is an $m \in \mathbb{N}_{>0}^{\oplus S_{K}}$ with $C_{K}^{m} \subset N$.

Suppose that $K$ is orientable and closed. Let $m=\left(m_{\wp}\right)_{\wp \in S_{K}} \in \mathbb{N}_{>0}^{\oplus S_{K}}$. We put

$$
I_{K}^{(m)}:=\left(\prod_{\wp \in s_{m}} U_{\wp}^{\left(m_{\wp}\right)}\right) \times\left(\prod_{\wp \in S_{K} \backslash s_{m}} K_{\wp}^{\times}\right) \subset I_{K} .
$$

By definition, we have $\prod_{\wp \in S_{K}} \widehat{\mathrm{B}}_{\mathrm{m}}^{1}\left(K_{\wp}\right) \subset I_{K}^{(m)}$.

Proposition 3.25 If $\left\{\right.$ Frob $\left._{\wp} \mid \wp \in S_{K} \backslash s_{m}\right\}$ generates $\mathrm{H}_{1}\left(M \backslash \operatorname{im}\left(s_{m}\right)\right)$, then the equality $I_{K}=I_{K}^{(m)}+P_{K}$ holds.

Proof We put $I:=\left\{\left(c_{\wp}\right)_{\wp \in S_{K}} \in I_{K} \mid\left(c_{\wp}\right)_{\wp \in s_{m}} \in \prod_{\wp \in s_{m}} \widehat{\mathrm{B}}_{\mathrm{m}}^{1}\left(K_{\wp}\right)\right\}$. By $I \subset I_{K}^{(m)}$, it suffices to verify $I_{K}=I+P_{K}$. By the assumption, there is $s^{\prime} \in \operatorname{Fin}\left(S_{K}\right)$ with $s^{\prime} \cap s_{m}=\varnothing$ such that $\left\{\operatorname{Frob}_{\wp} \mid \wp \in s^{\prime}\right\}$ generates $\mathrm{H}_{1}\left(M \backslash \operatorname{im}\left(s_{m}\right)\right)$, because $\mathrm{H}_{1}\left(M \backslash \operatorname{im}\left(s_{m}\right)\right)$ is a finitely generated Abelian group. We put $s:=s^{\prime} \sqcup s_{m}$. Replacing $s^{\prime}$ by a larger one, we can assume that $O_{K}\left[s^{-1}\right]$ is strict.

For each $\wp \in s$, we take a tubular neighbourhood $j_{\wp} \in \operatorname{Hom}_{\mathscr{C}}\left(O_{K}, \mathscr{A}_{V\left[r_{\wp}\right)}\right)$ with $r_{\wp} \in(0,1]$ of $\wp$. Replacing $\left(r_{\wp}\right)_{\wp \in s}$ by a family of smaller ones, we can assume that $\left(\operatorname{im}\left(j_{\wp}\right)\right)_{\wp \in s}$ is pairwise disjoint. We put $X \subset \mathscr{F}_{0}\left(O_{k}\right) \backslash \bigsqcup_{\wp \in s_{m}} \mathscr{F}\left(j_{\wp}\right)\left(V\left[2^{-1} r_{\wp}\right)\right)$ and $X_{0}:=M \backslash \bigsqcup_{\wp \in s} \mathscr{F}\left(j_{\wp}\right)\left(V\left[2^{-1} r_{\wp}\right)\right) \subset X$. We denote by $\varphi$ the group homomorphism

$$
\begin{aligned}
I_{K} \longrightarrow & H_{1}\left(\partial X_{0}\right), \\
\left(c_{\wp}\right)_{\wp \in S_{K}} \longmapsto & \sum_{\wp \in S} c_{\wp}\left(\lambda_{r_{\wp}, 2^{-1} r_{\wp}} \circ j_{\wp}\right)\left[\mathscr{F}\left(\mu_{r_{\wp}, 2^{-1} r_{\wp}} \circ j_{\wp}\right)\right] \\
& -\sum_{\wp \in S} c_{\wp}\left(\mu_{r_{\wp}, 2^{-1} r_{\wp}} \circ j_{\wp}\right)\left[\mathscr{F}\left(\lambda_{r_{\wp}, 2^{-1} r_{\wp}} \circ j_{\wp}\right)\right] .
\end{aligned}
$$

Then by the definition of $\widetilde{\rho}_{s_{m}}$, the restriction of $\widetilde{\rho}_{s_{m}}$ to $U:=\prod_{\wp \in S} K_{\wp}^{\times} \times \prod_{\wp \in S_{K} \backslash s} U_{\wp}$ coincides with the composite of the restriction of $\varphi$ to $U$ and the group homomorphism $\mathrm{H}_{1}\left(\partial X_{0}\right) \rightarrow \mathrm{H}_{1}\left(M \backslash \operatorname{im}\left(s_{m}\right)\right)$ associated with the inclusion $\partial X_{0} \rightarrow M \backslash \operatorname{im}\left(s_{m}\right)$.

Let $c=\left(c_{\wp}\right)_{\wp \in S_{K}} \in I_{K}$. We show $c \in I+P_{K}$. Replacing $s^{\prime}$ by a larger one, we can assume $c \in U$. By the choice of $s$, there is an $\left(n_{\wp}\right)_{\wp \in s^{\prime}} \in \mathbb{Z}^{\oplus s^{\prime}}$ with $\sum_{\wp \in s^{\prime}} n_{\wp}$ Frob $\tilde{\rho}_{s_{m}}(c) \in \mathrm{H}_{1}\left(M \backslash \operatorname{im}\left(s_{m}\right)\right)$. We put $\lambda:=\sum_{\wp \in s^{\prime}} n_{\wp}\left[\mathscr{F}\left(\lambda_{r_{\wp}, 2^{-1} r_{\wp}} \circ j_{\wp}\right)\right] \in \mathrm{H}_{1}\left(\partial X_{0}\right)$. We denote by $c^{\prime} \in \mathrm{H}_{1}\left(X_{0}\right)$ the image of $\varphi(c)-\lambda$ by the group homomorphism

$$
\mathrm{H}_{1}\left(\partial X_{0}\right) \longrightarrow \mathrm{H}_{1}\left(X_{0}\right)
$$

associated with the inclusion $\partial X_{0} \rightarrow X_{0}$. Then $c^{\prime}$ lies in the kernel of the group homomorphism $\mathrm{H}_{1}\left(X_{0}\right) \rightarrow \mathrm{H}_{1}(X)$ associated with the inclusion $X_{0} \rightarrow X$, because the group homomorphism $\mathrm{H}_{1}(X) \rightarrow \mathrm{H}_{1}\left(M \backslash \mathrm{im}\left(s_{m}\right)\right)$ associated with the inclusion 
$X \hookrightarrow M \backslash \operatorname{im}\left(s_{m}\right)$ is an isomorphism. By the standard argument using the MayerVietoris exact sequence, there is an $\left(n_{\wp}^{\prime}\right)_{\wp \in s^{\prime}} \in \mathbb{Z}^{\oplus s^{\prime}}$ with $\sum_{\wp \in s^{\prime}} n_{\wp}^{\prime}\left[\mathscr{F}\left(\mu_{r_{\wp}, 2^{-1} r_{\wp}} \circ j_{\wp}\right)\right]=$ $c^{\prime} \in \mathrm{H}_{1}\left(X_{0}\right)$. We put $\mu:=\sum_{\wp \in s^{\prime}} n_{\wp}^{\prime}\left[\mathscr{F}\left(\mu_{r_{\wp}, 2^{-1} r_{\wp}} \circ j_{\wp}\right)\right] \in \mathrm{H}_{1}\left(\partial X_{0}\right)$. Then $\varphi(c)-\lambda-\mu$ lies in the kernel of the group homomorphism $\mathrm{H}_{1}\left(\partial X_{0}\right) \rightarrow \mathrm{H}_{1}\left(X_{0}\right)$ associated with the inclusion $\partial X_{0} \hookrightarrow X_{0}$.

We consider the diagram in the proof of Lemma 3.22 corresponding to $X_{0}$. By the exactness of the right column, there is a $\Sigma \in \mathrm{H}_{2}\left(X_{0}, \partial X_{0}\right)$ with $\partial \Sigma=\varphi(c)-\lambda-\mu$. We denote by $\sigma \in \mathrm{H}^{1}(X)$ the preimage of $\Sigma$ by the group isomorphism in the top vertical arrow. We take a $\widetilde{c} \in \mathrm{Z}_{\mathrm{m}}^{1}\left(O_{K}\left[s^{-1}\right]\right)$ whose image by the canonical projection $\mathrm{Z}_{\mathrm{m}}^{1}\left(O_{k}\left[s^{-1}\right]\right) \rightarrow \mathrm{H}_{\mathrm{m}}^{1}\left(O_{k}\left[s^{-1}\right]\right)$ coincides with the image of $\sigma$ by the composite of the inverse of the group isomorphism $\mathrm{H}^{1}(M \backslash \mathrm{im}(s)) \rightarrow \mathrm{H}^{1}(X)$ associated with the inclusion $X \hookrightarrow M \backslash \operatorname{im}(s)$ and the group isomorphism $\mathrm{H}^{1}(M \backslash \operatorname{im}(s)) \rightarrow \mathrm{H}_{\mathrm{m}}^{1}\left(O_{K}\left[s^{-1}\right]\right)$ in Proposition 2.4. We denote by $c^{\prime} \in K^{\times}$the image of $\vec{c}$.

By the commutativity of the diagram up to signature, one of $c-\iota_{K}^{I}\left(c^{\prime}\right)$ and $c+\iota_{K}^{I}\left(c^{\prime}\right)$ lies in the preimage of $\lambda+\mu \in \mathrm{H}_{1}\left(\partial X_{0}\right)$ by $\varphi$, and hence in $I$, because $\lambda+\mu$ lies in the image of the group homomorphism $\mathrm{H}_{1}\left(\left(\partial X_{0}\right) \backslash(\partial X)\right) \rightarrow \mathrm{H}_{1}\left(\partial X_{0}\right)$ associated with the inclusion $\left(\partial X_{0}\right) \backslash(\partial X) \rightarrow \partial X_{0}$. It implies $c \in I+P_{K}$.

Remark 3.26 Proposition 3.25 is obviously an analogue of the approximation theorem in number theory [Neu99, \$II 3 (3.4)]. Moreover, by its proof, we have a much more accurate approximation in arithmetic topology than we ever have in number theory. Such difference between arithmetic topology and number theory originates from the fact that $\mathrm{H}_{\mathrm{m}}^{1}\left(K_{\wp}\right)$ with $\wp \in S_{K}$ is algebraically generated by two elements, while the multiplicative group of a local field is just topologically generated by two elements.

Suppose that $K$ is generic. Then we have $I_{K}=I_{K}^{(m)}+P_{K}$ by Proposition 3.25. By $I_{K}^{(m)} \subset \prod_{\wp \in s_{m}} U_{\wp} \times \prod_{\wp \in S_{K} \backslash s_{m}} K_{\wp}^{\times}$, the surjective group homomorphism $v_{K}: I_{K} \rightarrow \mathbb{Z}^{\oplus S_{K}}$ yields a surjective group homomorphism $v_{K}^{m}: I_{K}^{(m)} \rightarrow J_{K}^{m}$.

Theorem 3.27 (Ideal-Theoretic Class Field Theory) The group homomorphism $v_{K}^{m}$ induces a group isomorphism $C_{K} / C_{K}^{m} \rightarrow \mathrm{Cl}_{K}^{m}$ through the isomorphism

$$
C_{K}=\left(I_{K}^{(m)}+P_{K}\right) / P_{K} \cong I_{K}^{(m)} /\left(I_{K}^{(m)} \cap P_{K}\right) .
$$

Proof Let $c=\left(c_{\wp}\right)_{\wp \in S_{K}} \in I_{K}^{(m)} \cap P_{K}$. We take a $c^{\prime} \in K^{\times}$with $\iota_{K}^{I}\left(c^{\prime}\right)=c$. Then we have $\iota_{\wp}^{\times}\left(c^{\prime}\right)=c_{\wp} \in U_{\wp}^{\left(m_{\wp}\right)}$ for any $\wp \in s_{m}$. By the definition of $\iota_{K}^{m}$ and $v_{K}^{m}$, we obtain $v_{K}^{m}(c)=v_{K}(c)=\left(v_{\wp}\left(c_{\wp}\right)\right)_{\wp \in S_{K}}=\left(v_{\wp}\left(l_{\wp}^{\times}\left(c^{\prime}\right)\right)\right)_{\wp \in S_{K}} \in P_{K}^{m}$. Therefore $v_{K}^{m}$ induces a group homomorphism $\varphi: C_{K} \rightarrow \mathrm{Cl}_{K}^{m}$.

By $I_{K}^{m}=I_{K}^{(m)} \cap U_{K}=I_{K}^{(m)} \cap \operatorname{ker}\left(v_{K}\right), C_{K}^{m}$ is contained in $\operatorname{ker}(\varphi)$. Let $\bar{c} \in \operatorname{ker}(\varphi)$. We take a representative $c=\left(c_{\wp}\right)_{\wp \in S_{K}} \in I_{K}$ of $\bar{c}$. Then we have $v_{K}^{m}(c) \in P_{K}^{m}$, and hence there is a $c^{\prime} \in K^{\times}$with $\iota_{\wp}^{\times}\left(c^{\prime}\right) \in U_{\wp}^{\left(m_{\wp}\right)}$ for any $\wp \in s_{m}$ and $v_{\wp}\left(\iota_{\wp}\left(c^{\prime}\right)\right)=v_{\wp}\left(c_{\wp}\right)$ for any $\wp \in S_{K}$. It implies $\iota_{K}^{I}\left(c^{\prime}\right) \in I_{K}^{(m)}$ and $c-\iota_{K}^{I}\left(c^{\prime}\right) \in \operatorname{ker}\left(v_{K}^{m}\right)+P_{K}=I_{K}^{m}+P_{K}$. We obtain $\bar{c}=\left(\left(c-\iota_{K}^{I}\left(c^{\prime}\right)\right)+\iota_{K}^{I}\left(c^{\prime}\right)\right)+P_{K} \in C_{K}^{m}$. As a consequence, $\varphi$ induces a group isomorphism $C_{K} / C_{K}^{m} \rightarrow \mathrm{Cl}_{K}^{m}$. 
We say that $K$ is an arithmetic $\mathbb{Q} H S^{3}$ if $M$ is a rational homology sphere as a topological manifold.

Proposition 3.28 If $K$ is an orientable closed generic arithmetic $\mathbb{Q H S}{ }^{3}$, then the following assertions hold.

(i) For any $m \in \mathbb{N}_{>0}^{\oplus S_{K}}, C_{K}^{m} \subset C_{K}$ is an open subgroup of finite index.

(ii) For any open subgroup $N \subset C_{K}$ offinite index, there is an $m \in \mathbb{N}_{>0}^{\oplus S_{K}}$ with $C_{K}^{m} \subset N$.

Proof By Proposition 3.24, it suffices to verify that $C_{K}^{m} \subset C_{K}$ is of finite index for any $m \in \mathbb{N}_{>0}^{\oplus S_{K}}$. By Remark 2.10 and Theorem 3.27, we have a natural group iso-

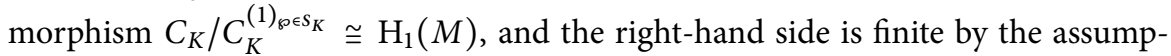
tion. Moreover, we have natural group isomorphisms $C_{K}^{(1)_{\wp} \in S_{K}} / C_{K}^{m} \cong I_{K}^{(1)_{\wp \in S_{K}}} / I_{K}^{m} \cong$ $\prod_{\wp \in s_{m}}\left(O_{K}\right)_{\wp}^{\times} / U_{\wp}^{\left(m_{\wp}\right)} \cong \prod_{\wp \in s_{m}} \mathbb{Z} / m_{\wp} \mathbb{Z}$. Thus we obtain

$$
\#\left(C_{K} / C_{K}^{m}\right)=\#\left(C_{K} / C_{K}^{(1)_{\wp} \in S_{K}}\right) \times \#\left(C_{K}^{(1)_{\wp}{ }^{i} n s_{K}} / C_{K}^{m}\right)=\# \mathrm{H}_{1}(M) \times \prod_{\wp \in s_{m}} m_{\wp}<\infty .
$$

Suppose that $K$ is an orientable closed stably generic arithmetic $\mathbb{Q} H S^{3}$. For an $m \in \mathbb{N}_{>0}^{\oplus S_{K}}$, we denote by $K^{m} \in M^{\mathrm{Ab}}(K)$ the element with $N_{K^{m} / K}=C_{K}^{m}$ that uniquely exists by Theorem 3.23 (ii) and Proposition 3.28 (i). As a consequence of Proposition 3.28 (ii), we obtain the following.

Theorem 3.29 (Kronecker-Weber theorem) If $K$ is an orientable closed stably generic arithmetic $\mathbb{Q H S}{ }^{3}$, then for any $L \in M^{\mathrm{Ab}}(K)$, there is an $m \in \mathbb{N}_{>0}^{\oplus S_{K}}$ with $L \leq K^{m}$.

\section{A Appendix: Existence of a Stably Generic Link}

We construct a stably generic link, in order to show that our class field theory is not nonsense.

Theorem A.1 Let $M$ be an orientable closed three-dimensional strict $\mathscr{C}$-manifold. Then there is a countable stably generic link in $\mathscr{A}_{M}$.

In order to verify Theorem A.1, it suffices to prove the following.

Lemma A.2 Let $O$ be a closed pre-arithmetic object of $\mathscr{C}_{0}$ in which there is a finite tame link s such that $O\left[s^{-1}\right]$ is an orientable arithmetic object of $\mathscr{C}_{0}$. Then there is a countable stably generic link in $O$.

Let $K$ be an arithmetic manifold. We put $M:=\mathscr{F}_{0}\left(O_{K}\right)$, and choose $\iota_{\infty} \in M \backslash$ $\operatorname{im}\left(S_{K}\right)$. In order to verify Lemma A.2, we prepare three lemmata.

Lemma A.3 If $S_{K}$ is finite and $K$ is an orientable closed arithmetic manifold, then for any $L \in \mathrm{ob}\left(\mathscr{C}_{0} / K\right)$, there is a finite tame link $s$ in $O_{K}$ containing $S_{K}$ such that $h_{L / K}^{*}\left(s \backslash S_{K}\right)$ forms an admissible link in $O_{L}\left[S_{L}^{-1}\right]$. 
Lemma A.4 If $S_{K}$ is countable and $K$ is closed, then there is a countable subset $C \subset \mathrm{ob}\left(\mathscr{C}_{0} / K\right)$ such that every object of $\mathscr{C}_{0} / K$ is isomorphic to an element of $C$ in $\mathscr{C}_{0} / K$.

Lemma A.5 If $S_{K}$ is finite and $K$ is an orientable closed arithmetic manifold, then there is a countable stably generic link in $O_{K}$ containing $S_{K}$.

Proof of Lemma A.3. By the assumption, $L$ is an orientable closed arithmetic manifold. By the finiteness of $S_{K}, S_{L}$ is also finite because its cardinality is bounded by \# $S_{K}$ multiplied by the degree of the unbranched covering associated with $h_{L / K}$. We put $V:=\mathscr{F}_{0}\left(O_{L}\right) \mathrm{im}\left(S_{L}\right)$, and take an orientable strict $\mathscr{C}$-manifold $Y$ with $\mathscr{A}_{Y}=\left.O_{L}\right|_{V}$. Then $\mathrm{H}_{1}(V)$ is a finitely generated Abelian group. By a standard smoothing argument, there is a finite set $\mathscr{L}$ of injective maps $S^{1} \hookrightarrow Y$ of class $\mathrm{C}^{\infty}$ with pairwise disjoint images such that the set of homology classes of 1-cycles $\{\gamma \circ \theta \mid \gamma \in \mathscr{L}\}$ generates $\mathrm{H}_{1}(V)$ and $h_{L / K} \circ \chi$ gives a tame cycle in $O_{K}\left[S_{K}^{-1}\right]$ for any $\chi \in \mathscr{L}$. Then the union of $S_{K}$ and the set of knots represented by $h_{L / K} \circ \chi$ with $\chi \in \mathscr{L}$ forms a desired finite tame link in $O_{K}$.

Proof of Lemma A.4. It suffices to show that $M(K)$ is countable. Let $s \in \operatorname{Fin}\left(S_{K}\right)$. Since $O_{K}\left[s^{-1}\right]$ is pre-admissible, $\pi_{1}(M \backslash \operatorname{im}(s))$ is a finitely generated group, and hence admits at most countably many subgroups of finite index. It implies that

$$
\operatorname{Cov}\left(M \backslash \operatorname{im}(s), \iota_{\infty}\right)
$$

is countable. Therefore, $\operatorname{Cov}(K)$ is countable, because so is $S_{K}$. Thus $M(K)$ is countable.

Proof of Lemma A.5. Since $O_{K}$ admits a tame knot, we can assume $S_{K} \neq \varnothing$. We denote by $\Sigma$ the set of finite tame links in $O_{K}$ containing $S_{K}$. We construct an increasing sequence $\left(s_{i}\right)_{i \in \mathbb{N}} \in \Sigma^{\mathbb{N}}$ and an $\left(\left(L_{i, j}\right)_{j \in \mathbb{N}}\right)_{i \in \mathbb{N}} \in \prod_{i \in \mathbb{N}} \mathrm{ob}\left(\mathscr{C}_{0} /\left(O_{K}, s_{i}\right)\right)^{\mathbb{N}}$ such that for any $i \in \mathbb{N}$, every object of $\mathscr{C}_{0} /\left(O_{K}, s_{i}\right)$ is isomorphic to $L_{i, j}$ in $\mathscr{C}_{0} /\left(O_{K}, s_{i}\right)$ for some $j \in \mathbb{N}$, and for any $(i, j, k) \in \mathbb{N}^{3}, h_{L_{i, j} /\left(O_{K}, s_{i}\right)}\left(s_{2^{i}\left(2\left(2^{j}(2 k+1)-1\right)+1\right)} \backslash s_{i+k}\right)$ forms an admissible link in $O_{L_{i, j}}\left[h_{L_{i, j} /\left(O_{K}, s_{i}\right)}^{*}\left(s_{i+k}\right)^{-1}\right]$.

Let $i \in \mathbb{N}$. Suppose that $s_{i^{\prime}}$ and $\left(L_{i^{\prime}, j}\right)_{j \in \mathbb{N}}$ have already been given for each $i^{\prime} \in$ $\mathbb{N} \cap[0, i-1]$. If $i=0$, then we put $s_{0}=S_{K}$. Suppose $i \neq 0$. We put $l:=\max \{e \in \mathbb{N} \mid$ $\left.2^{-e} i \in \mathbb{Z}\right\}, m:=\max \left\{e \in \mathbb{N} \mid 2^{-e}\left(2^{-1}\left(2^{-l} i-1\right)+1\right) \in \mathbb{Z}\right\}$, and $n:=2^{-1}\left(2^{-m}\left(2^{-1}\left(2^{-l} i-\right.\right.\right.$ $1)+1)-1)$. Then we have $n \in \mathbb{N}$ and $l+n<2^{l}(2 n+1) \leq i$. By Lemma A.3 applied to $\left(O_{K}, s_{i-1}\right)$ and $\left(O_{L_{l, m}}, h_{L_{l, m} /\left(O_{K}, s_{l}\right)}^{*}\left(s_{i-1}\right)\right)$, there is an $s_{i} \in \Sigma$ with $s_{i-1} \subset s_{i}$ such that

$$
h_{L_{l, m} /\left(O_{K}, s_{l}\right)}^{*}\left(s_{i} \backslash s_{i-1}\right)
$$

forms an admissible link in $O_{L_{l, m}}\left[h_{L_{l, m} /\left(O_{K}, s_{l}\right)}^{*}\left(s_{i-1}\right)^{-1}\right]$. By a standard argument using the Mayer-Vietoris exact sequence, the group homomorphism

$$
\begin{aligned}
\mathrm{H}_{1}\left(\mathscr{F}_{0}\left(O_{L_{l, m}}\right) \backslash \operatorname{im}\left(h_{L_{l, m} /\left(O_{K}, s_{l}\right)}^{*}\left(s_{i-1}\right)\right)\right) & \\
& \longrightarrow \mathrm{H}_{1}\left(\mathscr{F}_{0}\left(O_{L_{l, m}}\right) \backslash \operatorname{im}\left(h_{L_{l, m} /\left(O_{K}, s_{l}\right)}^{*}\left(s_{l+n}\right)\right)\right)
\end{aligned}
$$

associated with the inclusion is surjective. Therefore,

$$
\left\{\operatorname{Frob}_{\mathscr{P}} \mid \mathscr{P} \in h_{L_{l, m} /\left(O_{K}, s_{l}\right)}^{*}\left(s_{i} \backslash s_{l+n}\right)\right\}
$$


generates $\left.\mathrm{H}_{1}\left(\mathscr{F}_{0}\left(O_{L_{l, m}}\right) \backslash \operatorname{im}\left(h_{L_{l, m} /\left(O_{K}, s_{l}\right)}^{*}\left(s_{l+n}\right)^{-1}\right]\right)\right)$.

We have constructed $s_{i} \in \Sigma$. By Lemma A.4 applied to $\left(O_{K}, s_{i}\right)$, there is an

$$
\left(L_{i, j}\right)_{j \in \mathbb{N}} \in \mathrm{ob}\left(\mathscr{C}_{0} /\left(O_{K}, s_{i}\right)\right)^{\mathbb{N}}
$$

such that every object of $\mathscr{C}_{0} /\left(O_{K}, s_{i}\right)$ is isomorphic to $L_{i, j}$ in $\mathscr{C}_{0} /\left(O_{K}, s_{i}\right)$ for some $j \in \mathbb{N}$. By the induction on $i$, we obtain a desired pair of $\left(s_{i}\right)_{i \in \mathbb{N}}$ and $\left(L_{i, j}\right)_{(i, j) \in \mathbb{N}^{2}}$. Then $\cup_{i \in \mathbb{N}} s_{i}$ is a stably generic link in $O_{K}$ containing $S_{K}$ by the construction.

Proof of Lemma A.2. The assertion follows from Lemma A.5 applied to $(O, s)$ for a finite tame link $s$ in $O$ such that $O\left[s^{-1}\right]$ is an orientable arithmetic object of $\mathscr{C}_{0}$.

Acknowledgements I am deeply grateful to Masanori Morishita, who gave me an opportunity to study arithmetic topology. I am extremely thankful to Atsushi Yamashita for instructing me on elementary facts on low-dimensional topology. I could not have written several proofs in this paper without his help. I appreciate daily discussions with my colleagues. Personal communication with Hirofumi Niibo and Jun Ueki contributed most greatly to my work in this paper. I would like to thank my family for their deep affection. I was a research fellow of JST CREST, and was a research fellow of Japan Society for the Promotion of Science.

\section{References}

[Den02] C. Deninger, A Note on arithmetical topology and dynamical systems. In: Algebraic number theory and algebraic geometry. Contemp. Math., 300. Amer. Math. Soc., Providence, RI, 2002, pp. 99-114. http://dx.doi.org/10.1090/conm/300/05144

[Fox57] R. H. Fox, Covering spaces with singularities. In: A symposium in honor of S. Lefschetz. Princeton University Press, Princeton, NJ, 1957, pp. 243-257.

[Hir76] M. W. Hirsch, Differential topology. Graduate Texts in Mathematics, 33. Springer-Verlag, New York, 1976.

[Lee03] J. M. Lee, Introduction to smooth manifolds. Graduate Texts in Mathematics, 218. Springer-Verlag, new York, 2003. http://dx.doi.org/10.1007/978-0-387-21752-9

[Mor01] M. Morishita Knots and prime numbers, 3-dimensional manifolds and algebraic number fields. In: Algebraic number theory and related topics. Sūrikaisekikenkyūsho Kôkyûroku (2001), no. 1200, 103-115.

[Mor12] $\longrightarrow$ Knots and primes. An introduction to arithmetic topology. Universitext, Springer, London, 2012. http://dx.doi.org/10.1007/978-1-4471-2158-9

[Neu99] J. Neukirch, Algebraic number theory. Grundlehren der Mathematischen Wissenschaften, 322. Springer-Verlag, Berlin, 1999. http://dx.doi.org/10.1007/978-3-662-03983-0

[NU] H. Niibo and J. Ueki, Idelic class field theory for 3-manifolds and very admissible links. Trans. Amer. Math. Soc., to appear.

Tsukuba University, 1-1-1 Tennodai, Tsukuba, Ibaraki 305-8577, Japan

Email: mihara@math.tsukuba.ac.jp 